\title{
Crystal Structures and Spectroscopic Characterization of Radical Cations and Dications of Oligothiophenes Stabilized by Annelation with Bicyclo[2.2.2]octene Units: Sterically Segregated Cationic Oligothiophenes
}

Tohru Nishinaga, Atsushi Wakamiya, Daisuke Yamazaki, and Koichi Komatsu*

\section{Contents}

ORTEP drawing of the X-ray structure of $\mathbf{1}(2 \mathrm{~T})^{+} \mathrm{SbF}_{6}^{-}$(Figure S1) 5

Crystal data and structure refinement for $\mathbf{1}(2 \mathrm{~T})^{+} \mathrm{SbF}_{6}^{-}$(Table S1) 5

Atomic coordinates and equivalent isotropic displacement parameters for $\mathbf{1}(2 \mathrm{~T})^{+} \mathrm{SbF}_{6}^{-}$

(Table S2)

Bond lengths and angles for $\mathbf{1}(2 \mathrm{~T})^{+} \mathrm{SbF}_{6}{ }^{-}$(Table S3) $\quad 6$

ORTEP drawing of the X-ray structure of $\mathbf{1}(3 \mathrm{~T})^{+} \mathrm{SbF}_{6}^{-} \mathrm{CH}_{2} \mathrm{Cl}_{2}$ (Figure S2) 9

Crystal data and structure refinement for $\mathbf{1}(3 \mathrm{~T})^{+} \mathrm{SbF}_{6}{ }^{-} \mathrm{CH}_{2} \mathrm{Cl}_{2}$ (Table S4) 9

Atomic coordinates and equivalent isotropic displacement parameters for

$\mathbf{1}(3 \mathrm{~T})^{+} \mathrm{SbF}_{6}^{-} \mathrm{CH}_{2} \mathrm{Cl}_{2}$ (Table S5) 10

Bond lengths and angles for $\mathbf{1}(3 \mathrm{~T})^{+} \mathrm{SbF}_{6}{ }^{-} \mathrm{CH}_{2} \mathrm{Cl}_{2}$ (Table S6) 12

ORTEP drawing of the X-ray structure of $\mathbf{1}(4 \mathrm{~T})^{2+}\left(\mathrm{SbF}_{6}{ }^{-}\right)_{2}$ (Figure S3) 18

Crystal data and structure refinement for $\mathbf{1}(4 \mathrm{~T})^{2+}\left(\mathrm{SbF}_{6}{ }^{-}\right)_{2}$ (Table S7) 19

Atomic coordinates and equivalent isotropic displacement parameters for $\mathbf{1}(4 \mathrm{~T})^{2+}\left(\mathrm{SbF}_{6}^{-}\right)_{2}$

$\begin{array}{ll}\text { (Table S8) } & 20\end{array}$

Bond lengths and angles for $\mathbf{1}(4 \mathrm{~T})^{2+}\left(\mathrm{SbF}_{6}^{-}\right)_{2}$ (Table S9) 21

ORTEP drawing of the X-ray structure of $\mathbf{1}(6 \mathrm{~T})^{2+}\left(\mathrm{SbF}_{6}{ }^{-}\right)_{2} \mathrm{CH}_{2} \mathrm{Cl}_{2}$ (Figure S4) 25

Crystal data and structure refinement for $\mathbf{1}(6 \mathrm{~T})^{2+}\left(\mathrm{SbF}_{6}^{-}\right)_{2} \mathrm{CH}_{2} \mathrm{Cl}_{2}$ (Table S10) 26

Atomic coordinates and equivalent isotropic displacement parameters for

$\mathbf{1}(6 \mathrm{~T})^{2+}\left(\mathrm{SbF}_{6}^{-}\right)_{2} \mathrm{CH}_{2} \mathrm{Cl}_{2}($ Table S11) 27

Bond lengths and angles for $\mathbf{1}(6 \mathrm{~T})^{2+}\left(\mathrm{SbF}_{6}^{-}\right)_{2} \mathrm{CH}_{2} \mathrm{Cl}_{2}$ (Table S12) 29

Cartesian Coordinates from the Optimized Structure of $1(2 \mathrm{~T})^{++}$at B3LYP/6-31G(d)

(Table S13)

Cartesian Coordinates from the Optimized Structure of $1(2 \mathrm{~T})^{2+}$ (transoid)

$\begin{array}{ll}\text { at B3LYP/6-31G(d) (Table S14) } & 40\end{array}$ 
Cartesian Coordinates from the Optimized Structure of $\mathbf{1}(2 \mathrm{~T})^{2+}$ (cisoid)

at B3LYP/6-31G(d) (Table S15)

Cartesian Coordinates from the Optimized Structure of 1(3T) ${ }^{+}$at B3LYP/6-31G(d)

(Table S16)

Cartesian Coordinates from the Optimized Structure of $1(3 \mathrm{~T})^{2+}$ (transoid-transoid)

at B3LYP/6-31G(d) (Table S17)

Cartesian Coordinates from the Optimized Structure of $\mathbf{1}(3 \mathrm{~T})^{2+}$ (cisoid-transoid)

at B3LYP/6-31G(d) (Table S18)

Cartesian Coordinates from the Optimized Structure of $1(3 \mathrm{~T})^{2+}$ (cisoid-cisoid)

at B3LYP/6-31G(d) (Table S19)

Cartesian Coordinates from the Optimized Structure of $\mathbf{1}(4 \mathrm{~T})^{2+}$ at B3LYP/6-31G(d)

(Table S20)

Cartesian Coordinates from the Optimized Structure of bithiophene dication $\left(\mathrm{C}_{\mathrm{b}}-\mathrm{C}_{\mathrm{a}}-\mathrm{C}_{\mathrm{a}}-\mathrm{C}_{\mathrm{b}}=0^{\circ}\right)$ at B3LYP/6-31G(d) (Table S21)

Cartesian Coordinates from the Optimized Structure of bithiophene dication $\left(\mathrm{C}_{\mathrm{b}}-\mathrm{C}_{\mathrm{a}}-\mathrm{C}_{\mathrm{a}}-\mathrm{C}_{\mathrm{b}}=10^{\circ}\right)$ at B3LYP/6-31G(d) (Table S22)

Cartesian Coordinates from the Optimized Structure of bithiophene dication $\left(\mathrm{C}_{\mathrm{b}}-\mathrm{C}_{\mathrm{a}}-\mathrm{C}_{\mathrm{a}}-\mathrm{C}_{\mathrm{b}}=20^{\circ}\right)$ at B3LYP/6-31G(d) (Table S23)

Cartesian Coordinates from the Optimized Structure of bithiophene dication $\left(\mathrm{C}_{\mathrm{b}}-\mathrm{C}_{\mathrm{a}}-\mathrm{C}_{\mathrm{a}}-\mathrm{C}_{\mathrm{b}}=30^{\circ}\right)$ at B3LYP/6-31G(d) (Table S24)

Cartesian Coordinates from the Optimized Structure of bithiophene dication $\left(\mathrm{C}_{\mathrm{b}}-\mathrm{C}_{\mathrm{a}}-\mathrm{C}_{\mathrm{a}}-\mathrm{C}_{\mathrm{b}}=40^{\circ}\right)$ at B3LYP/6-31G(d) (Table S25)

Cartesian Coordinates from the Optimized Structure of bithiophene dication $\left(\mathrm{C}_{\mathrm{b}}-\mathrm{C}_{\mathrm{a}}-\mathrm{C}_{\mathrm{a}}-\mathrm{C}_{\mathrm{b}}=50^{\circ}\right)$ at B3LYP/6-31G(d) (Table S26)

Cartesian Coordinates from the Optimized Structure of bithiophene dication $\left(\mathrm{C}_{\mathrm{b}}-\mathrm{C}_{\mathrm{a}}-\mathrm{C}_{\mathrm{a}}-\mathrm{C}_{\mathrm{b}}=60^{\circ}\right)$ at B3LYP/6-31G(d) (Table S27)

Cartesian Coordinates from the Optimized Structure of bithiophene dication $\left(\mathrm{C}_{\mathrm{b}}-\mathrm{C}_{\mathrm{a}}-\mathrm{C}_{\mathrm{a}}-\mathrm{C}_{\mathrm{b}}=70^{\circ}\right)$ at B3LYP/6-31G(d) (Table S28)

Cartesian Coordinates from the Optimized Structure of bithiophene dication $\left(\mathrm{C}_{\mathrm{b}}-\mathrm{C}_{\mathrm{a}}-\mathrm{C}_{\mathrm{a}}-\mathrm{C}_{\mathrm{b}}=80^{\circ}\right)$ at B3LYP/6-31G(d) (Table S29)

Cartesian Coordinates from the Optimized Structure of bithiophene dication $\left(\mathrm{C}_{\mathrm{b}}-\mathrm{C}_{\mathrm{a}}-\mathrm{C}_{\mathrm{a}}-\mathrm{C}_{\mathrm{b}}=90^{\circ}\right)$ at B3LYP/6-31G(d) (Table S30) 
Cartesian Coordinates from the Optimized Structure of bithiophene dication $\left(\mathrm{C}_{\mathrm{b}}-\mathrm{C}_{\mathrm{a}}-\mathrm{C}_{\mathrm{a}}-\mathrm{C}_{\mathrm{b}}=100^{\circ}\right)$ at B3LYP/6-31G(d) (Table S31)

Cartesian Coordinates from the Optimized Structure of bithiophene dication $\left(\mathrm{C}_{\mathrm{b}}-\mathrm{C}_{\mathrm{a}}-\mathrm{C}_{\mathrm{a}}-\mathrm{C}_{\mathrm{b}}=110^{\circ}\right)$ at B3LYP/6-31G(d) (Table S32)

Cartesian Coordinates from the Optimized Structure of bithiophene dication $\left(\mathrm{C}_{\mathrm{b}}-\mathrm{C}_{\mathrm{a}}-\mathrm{C}_{\mathrm{a}}-\mathrm{C}_{\mathrm{b}}=120^{\circ}\right)$ at B3LYP/6-31G(d) (Table S33)

Cartesian Coordinates from the Optimized Structure of bithiophene dication $\left(\mathrm{C}_{\mathrm{b}}-\mathrm{C}_{\mathrm{a}}-\mathrm{C}_{\mathrm{a}}-\mathrm{C}_{\mathrm{b}}=130^{\circ}\right)$ at B3LYP/6-31G(d) (Table S34)

Cartesian Coordinates from the Optimized Structure of bithiophene dication $\left(\mathrm{C}_{\mathrm{b}}-\mathrm{C}_{\mathrm{a}}-\mathrm{C}_{\mathrm{a}}-\mathrm{C}_{\mathrm{b}}=140^{\circ}\right)$ at B3LYP/6-31G(d) (Table S35)

Cartesian Coordinates from the Optimized Structure of bithiophene dication $\left(\mathrm{C}_{\mathrm{b}}-\mathrm{C}_{\mathrm{a}}-\mathrm{C}_{\mathrm{a}}-\mathrm{C}_{\mathrm{b}}=150^{\circ}\right)$ at B3LYP/6-31G(d) (Table S36)

Cartesian Coordinates from the Optimized Structure of bithiophene dication $\left(\mathrm{C}_{\mathrm{b}}-\mathrm{C}_{\mathrm{a}}-\mathrm{C}_{\mathrm{a}}-\mathrm{C}_{\mathrm{b}}=160^{\circ}\right)$ at B3LYP/6-31G(d) (Table S37)

Cartesian Coordinates from the Optimized Structure of bithiophene dication $\left(\mathrm{C}_{\mathrm{b}}-\mathrm{C}_{\mathrm{a}}-\mathrm{C}_{\mathrm{a}}-\mathrm{C}_{\mathrm{b}}=170^{\circ}\right)$ at B3LYP/6-31G(d) (Table S38)

Cartesian Coordinates from the Optimized Structure of bithiophene dication $\left(\mathrm{C}_{\mathrm{b}}-\mathrm{C}_{\mathrm{a}}-\mathrm{C}_{\mathrm{a}}-\mathrm{C}_{\mathrm{b}}=180^{\circ}\right)$ at B3LYP/6-31G(d) (Table S39)

Cartesian Coordinates from the Optimized Structure of terthiophene dication $\left(\mathrm{C}_{\mathrm{b}}-\mathrm{C}_{\mathrm{a}}-\mathrm{C}_{\mathrm{a}}-\mathrm{C}_{\mathrm{b}}=0^{\circ}, \mathrm{C}_{\mathrm{b}}{ }^{\prime}-\mathrm{C}_{\mathrm{a}}{ }^{\prime}-\mathrm{C}_{\mathrm{a}}{ }^{\prime}-\mathrm{C}_{\mathrm{b}}{ }^{\prime}=180^{\circ}\right)$ at B3LYP/6-31G(d) (Table S40)

Cartesian Coordinates from the Optimized Structure of terthiophene dication $\left(\mathrm{C}_{\mathrm{b}}-\mathrm{C}_{\mathrm{a}}-\mathrm{C}_{\mathrm{a}}-\mathrm{C}_{\mathrm{b}}=30^{\circ}, \mathrm{C}_{\mathrm{b}}{ }^{\prime}-\mathrm{C}_{\mathrm{a}}{ }^{\prime}-\mathrm{C}_{\mathrm{a}}{ }^{\prime}-\mathrm{C}_{\mathrm{b}}{ }^{\prime}=180^{\circ}\right)$ at B3LYP/6-31G(d) (Table S41)

Cartesian Coordinates from the Optimized Structure of terthiophene dication $\left(\mathrm{C}_{\mathrm{b}}-\mathrm{C}_{\mathrm{a}}-\mathrm{C}_{\mathrm{a}}-\mathrm{C}_{\mathrm{b}}=60^{\circ}, \mathrm{C}_{\mathrm{b}}{ }^{\prime}-\mathrm{C}_{\mathrm{a}}{ }^{\prime}-\mathrm{C}_{\mathrm{a}}{ }^{\prime}-\mathrm{C}_{\mathrm{b}}{ }^{\prime}=180^{\circ}\right)$ at B3LYP/6-31G(d) (Table S42)

Cartesian Coordinates from the Optimized Structure of terthiophene dication $\left(\mathrm{C}_{\mathrm{b}}-\mathrm{C}_{\mathrm{a}}-\mathrm{C}_{\mathrm{a}}-\mathrm{C}_{\mathrm{b}}=90^{\circ}, \mathrm{C}_{\mathrm{b}}{ }^{\prime}-\mathrm{C}_{\mathrm{a}}{ }^{\prime}-\mathrm{C}_{\mathrm{a}}{ }^{\prime}-\mathrm{C}_{\mathrm{b}}{ }^{\prime}=180^{\circ}\right)$ at B3LYP/6-31G(d) (Table S43)

Cartesian Coordinates from the Optimized Structure of terthiophene dication $\left(\mathrm{C}_{\mathrm{b}}-\mathrm{C}_{\mathrm{a}}-\mathrm{C}_{\mathrm{a}}-\mathrm{C}_{\mathrm{b}}=120^{\circ}, \mathrm{C}_{\mathrm{b}}{ }^{\prime}-\mathrm{C}_{\mathrm{a}}{ }^{\prime}-\mathrm{C}_{\mathrm{a}}{ }^{\prime}-\mathrm{C}_{\mathrm{b}}{ }^{\prime}=180^{\circ}\right)$ at B3LYP/6-31G(d) (Table S44)

Cartesian Coordinates from the Optimized Structure of terthiophene dication $\left(\mathrm{C}_{\mathrm{b}}-\mathrm{C}_{\mathrm{a}}-\mathrm{C}_{\mathrm{a}}-\mathrm{C}_{\mathrm{b}}=150^{\circ}, \mathrm{C}_{\mathrm{b}}{ }^{\prime}-\mathrm{C}_{\mathrm{a}}{ }^{\prime}-\mathrm{C}_{\mathrm{a}}{ }^{\prime}-\mathrm{C}_{\mathrm{b}}{ }^{\prime}=180^{\circ}\right)$ at B3LYP/6-31G(d) (Table S45)

Cartesian Coordinates from the Optimized Structure of terthiophene dication $\left(\mathrm{C}_{\mathrm{b}}-\mathrm{C}_{\mathrm{a}}-\mathrm{C}_{\mathrm{a}}-\mathrm{C}_{\mathrm{b}}=180^{\circ}, \mathrm{C}_{\mathrm{b}}{ }^{\prime}-\mathrm{C}_{\mathrm{a}}{ }^{\prime}-\mathrm{C}_{\mathrm{a}}{ }^{\prime}-\mathrm{C}_{\mathrm{b}}{ }^{\prime}=180^{\circ}\right)$ at B3LYP/6-31G(d) (Table S46) 
Observed and Calculated ${ }^{13} \mathrm{C}$ NMR Chemical Shifts $(\delta)$ for $\mathbf{1}(2 \mathrm{~T})^{2+}$ and $\mathbf{1}(3 \mathrm{~T})^{2+}$

(Table S47) 

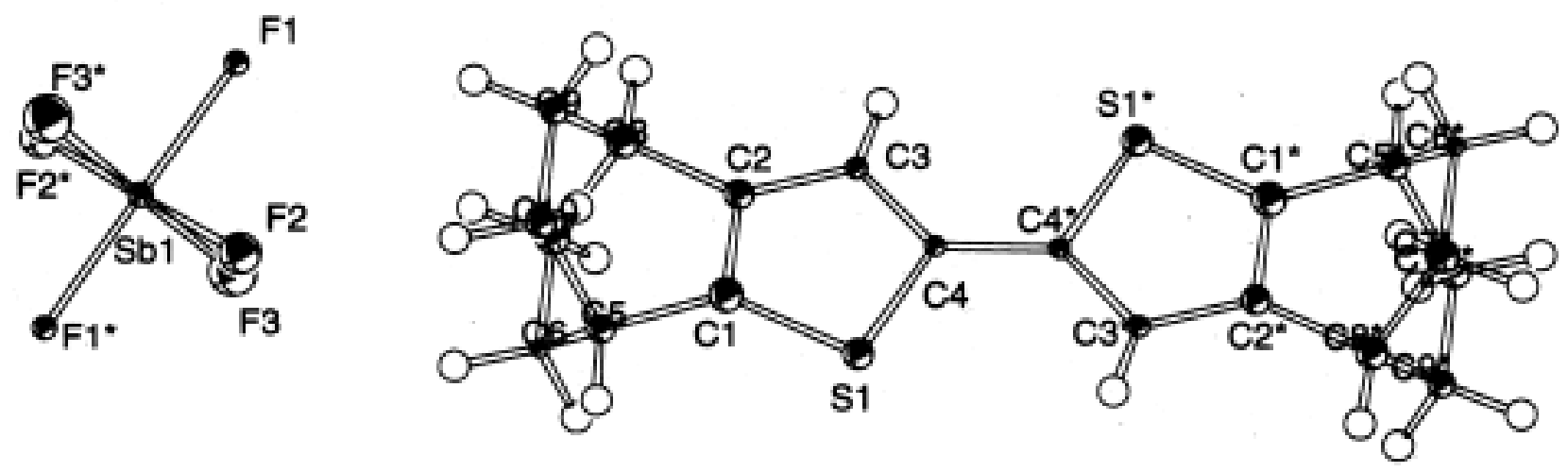

Figure S1. ORTEP drawing (50\% probability) showing the X-ray structure of $1(2 \mathrm{~T})^{+} \mathrm{SbF}_{6}^{-}$.

Table S1. Crystal data and structure refinement for $\mathbf{1}(2 \mathrm{~T})^{+} \mathrm{SbF}_{6}^{-}$.

Identification code

Empirical formula

Formula weight

Temperature

Wavelength

Crystal system

Space group

Unit cell dimensions

Volume

$\mathrm{Z}$

Density (calculated)

Absorption coefficient

$\mathrm{F}(000)$

Crystal size

Theta range for data collection

Index ranges

Reflections collected

Independent reflections

Completeness to theta $=24.99^{\circ}$

Refinement method
2 merc

C20 H22 F6 S2 Sb

562.25

100(2) K

$0.71069 \AA$

Triclinic

P-1

$$
\begin{array}{ll}
\mathrm{a}=7.680(5) \AA & \alpha=96.716(5)^{\circ} . \\
\mathrm{b}=8.211(5) \AA & \beta=113.151(5)^{\circ} . \\
\mathrm{c}=9.594(5) \AA & \gamma=107.298(5)^{\circ} .
\end{array}
$$

511.9(5) $\AA^{3}$

1

$1.824 \mathrm{Mg} / \mathrm{m}^{3}$

$1.609 \mathrm{~mm}^{-1}$

279

$0.15 \times 0.10 \times 0.05 \mathrm{~mm}^{3}$

2.40 to $24.99^{\circ}$.

$-9<=\mathrm{h}<=9,-4<=\mathrm{k}<=9,-11<=\mathrm{l}<=11$

2891

$1764[\mathrm{R}(\mathrm{int})=0.0266]$

$97.9 \%$

Full-matrix least-squares on $\mathrm{F}^{2}$ 
Data / restraints / parameters

Goodness-of-fit on F2

Final R indices [I $>2 \operatorname{sigma}(\mathrm{I})]$

$\mathrm{R}$ indices (all data)

Largest diff. peak and hole
1764 / 0 / 133

1.027

$$
\begin{aligned}
& \mathrm{R} 1=0.0345, \mathrm{wR} 2=0.0808 \\
& \mathrm{R} 1=0.0376, \mathrm{wR} 2=0.0820 \\
& 0.872 \text { and }-0.604 \mathrm{e} . \AA^{-3}
\end{aligned}
$$

\begin{tabular}{|c|c|c|c|c|}
\hline & $\mathrm{x}$ & $\mathrm{y}$ & $\mathrm{z}$ & $\mathrm{U}(\mathrm{eq})$ \\
\hline $\mathrm{Sb}(1)$ & 5000 & 0 & 10000 & $19(1)$ \\
\hline $\mathrm{S}(1)$ & $7626(2)$ & $5738(1)$ & $7308(1)$ & $23(1)$ \\
\hline $\mathrm{F}(1)$ & $6079(4)$ & $-1179(4)$ & $8963(3)$ & $36(1)$ \\
\hline $\mathrm{F}(3)$ & $5135(5)$ & $1667(4)$ & $8830(3)$ & $46(1)$ \\
\hline $\mathrm{F}(2)$ & $7637(4)$ & 1399(4) & $11560(3)$ & $40(1)$ \\
\hline $\mathrm{C}(2)$ & $6100(6)$ & $3241(6)$ & $4723(4)$ & $21(1)$ \\
\hline $\mathrm{C}(1)$ & $5761(6)$ & $4675(6)$ & $5362(4)$ & $19(1)$ \\
\hline $\mathrm{C}(3)$ & $7790(6)$ & $3019(6)$ & $5765(5)$ & $25(1)$ \\
\hline$C(5)$ & $10653(6)$ & $4139(6)$ & $8401(4)$ & $22(1)$ \\
\hline $\mathrm{C}(9)$ & $8886(7)$ & $834(6)$ & $7029(5)$ & $30(1)$ \\
\hline$C(10)$ & $9987(7)$ & $2259(6)$ & $8631(5)$ & $33(1)$ \\
\hline $\mathrm{C}(8)$ & $8803(6)$ & $1738(6)$ & $5697(5)$ & $23(1)$ \\
\hline$C(6)$ & $12119(7)$ & $4254(6)$ & $7650(5)$ & $33(1)$ \\
\hline$C(7)$ & $11028(7)$ & $2833(6)$ & $6051(5)$ & $28(1)$ \\
\hline $\mathrm{C}(4)$ & $8798(7)$ & $4300(6)$ & $7223(5)$ & $28(1)$ \\
\hline
\end{tabular}

Table S2. Atomic coordinates $\left(\mathrm{x} 10^{4}\right)$ and equivalent isotropic displacement parameters $\left(\AA^{2} \times 10^{3}\right)$ for $\mathbf{1}(2 \mathrm{~T})^{+} \mathrm{SbF}_{6}^{-}$. U(eq) is defined as one third of the trace of the orthogonalized Uij tensor.

Table S3. Bond lengths $[\AA]$ and angles $\left[{ }^{\circ}\right]$ for $\mathbf{1}(2 \mathrm{~T})^{+} \mathrm{SbF}_{6}^{-}$. 


\begin{tabular}{|c|c|}
\hline $\mathrm{Sb}(1)-\mathrm{F}(2)$ & $1.869(3)$ \\
\hline $\mathrm{Sb}(1)-\mathrm{F}(3)$ & $1.874(3)$ \\
\hline $\mathrm{Sb}(1)-\mathrm{F}(3) \# 1$ & $1.874(3)$ \\
\hline $\mathrm{Sb}(1)-\mathrm{F}(1) \# 1$ & $1.875(2)$ \\
\hline $\mathrm{Sb}(1)-\mathrm{F}(1)$ & $1.875(2)$ \\
\hline $\mathrm{S}(1)-\mathrm{C}(4)$ & $1.696(5)$ \\
\hline$S(1)-C(1)$ & $1.752(4)$ \\
\hline $\mathrm{C}(2)-\mathrm{C}(3)$ & $1.371(6)$ \\
\hline$C(2)-C(1)$ & $1.403(6)$ \\
\hline $\mathrm{C}(1)-\mathrm{C}(1) \# 2$ & $1.398(8)$ \\
\hline$C(3)-C(4)$ & $1.408(6)$ \\
\hline$C(3)-C(8)$ & $1.491(6)$ \\
\hline$C(5)-C(4)$ & $1.486(6)$ \\
\hline$C(5)-C(6)$ & $1.545(6)$ \\
\hline $\mathrm{C}(5)-\mathrm{C}(10)$ & $1.546(6)$ \\
\hline $\mathrm{C}(9)-\mathrm{C}(8)$ & $1.541(6)$ \\
\hline $\mathrm{C}(9)-\mathrm{C}(10)$ & $1.554(6)$ \\
\hline $\mathrm{C}(8)-\mathrm{C}(7)$ & $1.550(6)$ \\
\hline$C(6)-C(7)$ & $1.551(6)$ \\
\hline $\mathrm{F}(2) \# 1-\mathrm{Sb}(1)-\mathrm{F}(2)$ & $180.000(1)$ \\
\hline $\mathrm{F}(2) \# 1-\mathrm{Sb}(1)-\mathrm{F}(3)$ & $89.78(14)$ \\
\hline $\mathrm{F}(2)-\mathrm{Sb}(1)-\mathrm{F}(3)$ & $90.22(14)$ \\
\hline $\mathrm{F}(2) \# 1-\mathrm{Sb}(1)-\mathrm{F}(3) \# 1$ & $90.22(14)$ \\
\hline $\mathrm{F}(2)-\mathrm{Sb}(1)-\mathrm{F}(3) \# 1$ & $89.78(14)$ \\
\hline $\mathrm{F}(3)-\mathrm{Sb}(1)-\mathrm{F}(3) \# 1$ & $180.000(1)$ \\
\hline $\mathrm{F}(2) \# 1-\mathrm{Sb}(1)-\mathrm{F}(1) \# 1$ & $90.27(12)$ \\
\hline $\mathrm{F}(2)-\mathrm{Sb}(1)-\mathrm{F}(1) \# 1$ & $89.73(12)$ \\
\hline $\mathrm{F}(3)-\mathrm{Sb}(1)-\mathrm{F}(1) \# 1$ & $90.15(13)$ \\
\hline $\mathrm{F}(3) \# 1-\mathrm{Sb}(1)-\mathrm{F}(1) \# 1$ & $89.85(13)$ \\
\hline $\mathrm{F}(2) \# 1-\mathrm{Sb}(1)-\mathrm{F}(1)$ & $89.73(12)$ \\
\hline $\mathrm{F}(2)-\mathrm{Sb}(1)-\mathrm{F}(1)$ & $90.27(12)$ \\
\hline $\mathrm{F}(3)-\mathrm{Sb}(1)-\mathrm{F}(1)$ & $89.85(13)$ \\
\hline
\end{tabular}




$\begin{array}{ll}\mathrm{F}(3) \# 1-\mathrm{Sb}(1)-\mathrm{F}(1) & 90.15(13) \\ \mathrm{F}(1) \# 1-\mathrm{Sb}(1)-\mathrm{F}(1) & 180.000(1) \\ \mathrm{C}(4)-\mathrm{S}(1)-\mathrm{C}(1) & 90.5(2) \\ \mathrm{C}(3)-\mathrm{C}(2)-\mathrm{C}(1) & 112.7(3) \\ \mathrm{C}(1) \# 2-\mathrm{C}(1)-\mathrm{C}(2) & 128.7(4) \\ \mathrm{C}(1) \# 2-\mathrm{C}(1)-\mathrm{S}(1) & 120.0(4) \\ \mathrm{C}(2)-\mathrm{C}(1)-\mathrm{S}(1) & 111.3(3) \\ \mathrm{C}(2)-\mathrm{C}(3)-\mathrm{C}(4) & 112.4(4) \\ \mathrm{C}(2)-\mathrm{C}(3)-\mathrm{C}(8) & 134.5(4) \\ \mathrm{C}(4)-\mathrm{C}(3)-\mathrm{C}(8) & 113.1(4) \\ \mathrm{C}(4)-\mathrm{C}(5)-\mathrm{C}(6) & 106.8(3) \\ \mathrm{C}(4)-\mathrm{C}(5)-\mathrm{C}(10) & 107.0(4) \\ \mathrm{C}(6)-\mathrm{C}(5)-\mathrm{C}(10) & 107.7(4) \\ \mathrm{C}(8)-\mathrm{C}(9)-\mathrm{C}(10) & 109.7(4) \\ \mathrm{C}(5)-\mathrm{C}(10)-\mathrm{C}(9) & 110.7(3) \\ \mathrm{C}(3)-\mathrm{C}(8)-\mathrm{C}(9) & 107.8(3) \\ \mathrm{C}(3)-\mathrm{C}(8)-\mathrm{C}(7) & 107.0(4) \\ \mathrm{C}(9)-\mathrm{C}(8)-\mathrm{C}(7) & 108.0(4) \\ \mathrm{C}(5)-\mathrm{C}(6)-\mathrm{C}(7) & 110.2(4) \\ \mathrm{C}(8)-\mathrm{C}(7)-\mathrm{C}(6) & 110.3(3) \\ \mathrm{C}(3)-\mathrm{C}(4)-\mathrm{C}(5) & 115.0(4) \\ \mathrm{C}(3)-\mathrm{C}(4)-\mathrm{S}(1) & 113.1(3) \\ \mathrm{C}(5)-\mathrm{C}(4)-\mathrm{S}(1) & 131.9 \\ & \end{array}$

Symmetry transformations used to generate equivalent atoms:

$\# 1-\mathrm{x}+1,-\mathrm{y},-\mathrm{z}+2 \quad \# 2-\mathrm{x}+1,-\mathrm{y}+1,-\mathrm{z}+1$ 


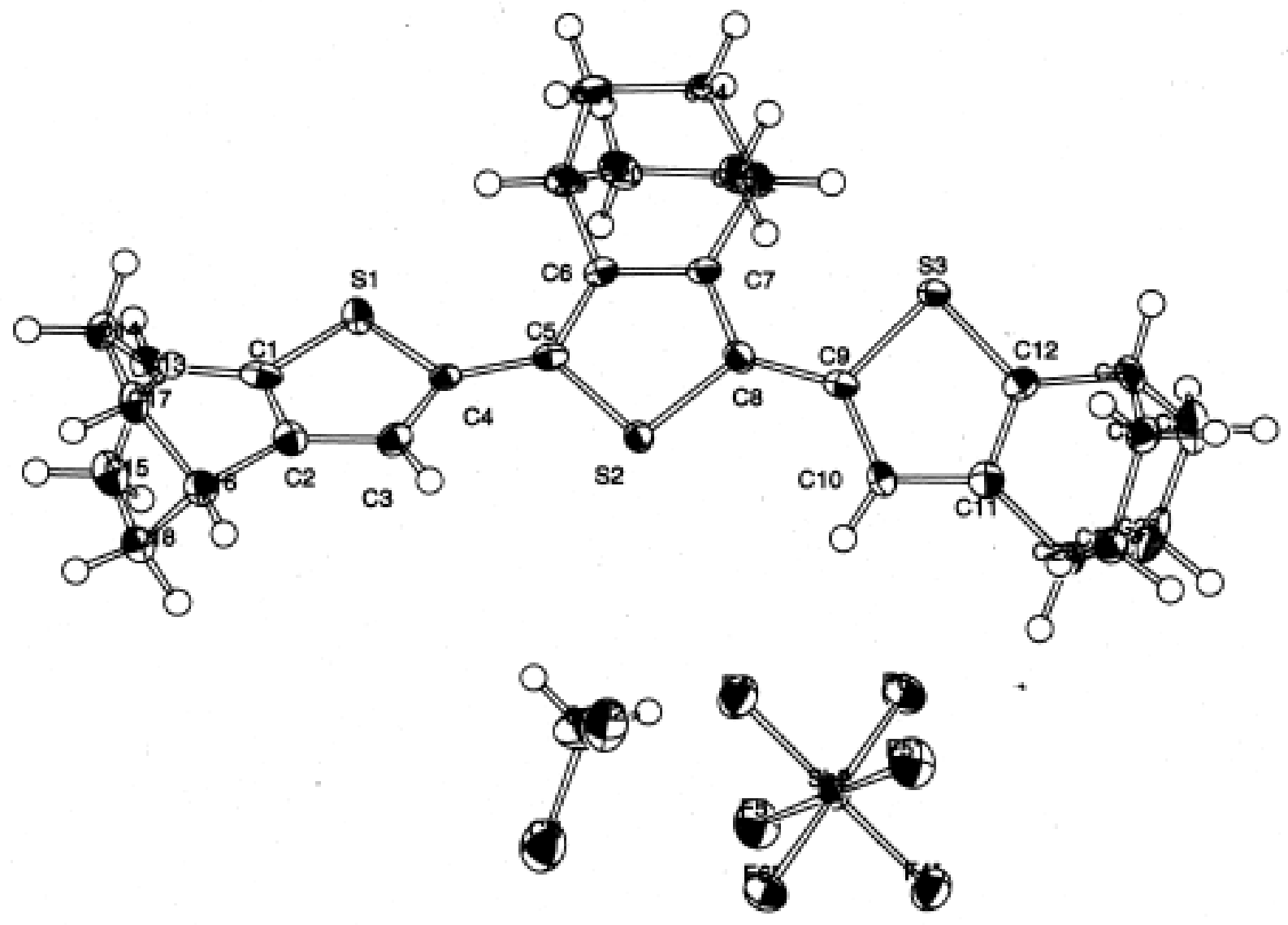

$\mathbf{F}$

Table S4. Crystal data and structure refinement for $1(3 \mathrm{~T})^{+} \mathrm{SbF}_{6}^{-} \mathrm{CH}_{2} \mathrm{Cl}_{2}$.

\section{Identification code}

Empirical formula

Formula weight

Temperature

Wavelength

Crystal system

Space group

Unit cell dimensions

Volume
3 merc

C31 H34 Cl2 F6 S3 Sb

809.41

$100(2) \mathrm{K}$

$0.71069 \AA$

Triclinic

P-1

$\mathrm{a}=9.653(5) \AA \quad \alpha=94.644(5)^{\circ}$.

$\mathrm{b}=12.687(5) \AA \quad \beta=100.024(5)^{\circ}$.

$\mathrm{c}=13.237(5) \AA \quad \gamma=98.039(5)^{\circ}$. 
Density (calculated)

Absorption coefficient

$\mathrm{F}(000)$

Crystal size

Theta range for data collection

Index ranges

Reflections collected

Independent reflections

Completeness to theta $=25.00^{\circ}$

Max. and min. transmission

Refinement method

Data / restraints / parameters

Goodness-of-fit on F2

Final $\mathrm{R}$ indices [I $>2 \operatorname{sigma}(\mathrm{I})]$

$\mathrm{R}$ indices (all data)

Largest diff. peak and hole
$1.711 \mathrm{Mg} / \mathrm{m}^{3}$

$1.306 \mathrm{~mm}^{-1}$

814

$0.20 \times 0.20 \times 0.20 \mathrm{~mm}^{3}$

2.14 to $25.00^{\circ}$.

$-11<=\mathrm{h}<=11,-15<=\mathrm{k}<=14,-15<=\mathrm{l}<=14$

7967

$5337[\mathrm{R}(\mathrm{int})=0.0238]$

$96.3 \%$

0.7802 and 0.7802

Full-matrix least-squares on $\mathrm{F}^{2}$

5337 / 36 / 429

1.085

$\mathrm{R} 1=0.0605, \mathrm{wR} 2=0.1803$

$\mathrm{R} 1=0.0672, \mathrm{wR} 2=0.1844$

4.876 and -0.901 e. $\AA^{-3}$

Table S5. Atomic coordinates ( x 104) and equivalent isotropic displacement parameters $\left(\AA^{2} \times 10^{3}\right)$ for $1(3 \mathrm{~T})^{+} \mathrm{SbF}_{6}^{-} \mathrm{CH}_{2} \mathrm{Cl}_{2}$. U(eq) is defined as one third of the trace of the orthogonalized Uij tensor.

\begin{tabular}{lllll}
\hline & $x$ & $\mathrm{z}$ & $\mathrm{U}(\mathrm{eq})$ \\
$\mathrm{S}(1)$ & & & \\
$\mathrm{C}(3)$ & $5614(3)$ & $12144(2)$ & $6447(2)$ & $19(1)$ \\
$\mathrm{S}(11)$ & $6682(15)$ & $10713(10)$ & $7482(9)$ & $23(3)$ \\
$\mathrm{C}(53)$ & $6910(20)$ & $10372(13)$ & $7631(10)$ & $18(4)$ \\
$\mathrm{S}(3)$ & $5800(60)$ & $11860(30)$ & $6720(20)$ & $25(12)$ \\
$\mathrm{C}(10)$ & $9544(3)$ & $8636(2)$ & $3034(2)$ & $20(1)$ \\
$\mathrm{S}(33)$ & $8010(14)$ & $7360(9)$ & $3986(10)$ & $19(2)$ \\
$\mathrm{C}(60)$ & $7740(20)$ & $7159(12)$ & $4242(16)$ & $12(4)$ \\
$\mathrm{Sb}(2)$ & $9400(50)$ & $8321(18)$ & $3300(40)$ & $16(12)$ \\
& 5000 & 5000 & 5000 & $17(1)$
\end{tabular}




\begin{tabular}{|c|c|c|c|c|}
\hline $\mathrm{Sb}(1)$ & 10000 & 10000 & 10000 & $24(1)$ \\
\hline$S(2)$ & $7318(2)$ & $9238(1)$ & $5571(1)$ & $21(1)$ \\
\hline $\mathrm{Cl}(2)$ & $8971(3)$ & $6684(2)$ & $8687(2)$ & $45(1)$ \\
\hline $\mathrm{Cl}(1)$ & $5859(3)$ & $6188(2)$ & $8479(2)$ & $46(1)$ \\
\hline $\mathrm{F}(4)$ & $5763(5)$ & $6397(3)$ & $5621(3)$ & $34(1)$ \\
\hline $\mathrm{F}(2)$ & $10976(5)$ & $9378(4)$ & $9058(3)$ & $43(1)$ \\
\hline $\mathrm{F}(6)$ & $5017(5)$ & $5491(3)$ & $3696(3)$ & $33(1)$ \\
\hline $\mathrm{F}(5)$ & $3157(4)$ & $5321(4)$ & $4967(4)$ & $37(1)$ \\
\hline $\mathrm{F}(1)$ & $8250(5)$ & $9278(4)$ & $9216(4)$ & $45(1)$ \\
\hline $\mathrm{F}(3)$ & $10177(5)$ & $8818(4)$ & $10763(3)$ & $37(1)$ \\
\hline$C(2)$ & $6223(7)$ & $11378(5)$ & $8215(5)$ & $21(1)$ \\
\hline$C(6)$ & $7138(7)$ & $10992(5)$ & $4697(5)$ & $16(1)$ \\
\hline $\mathrm{C}(1)$ & $5606(7)$ & $12179(5)$ & $7728(5)$ & $20(1)$ \\
\hline $\mathrm{C}(13)$ & $5066(7)$ & $12961(6)$ & $8412(5)$ & $24(2)$ \\
\hline$C(4)$ & $6461(6)$ & $11001(5)$ & $6495(5)$ & $15(1)$ \\
\hline$C(7)$ & $7737(7)$ & $10347(5)$ & $4050(5)$ & $18(1)$ \\
\hline $\mathrm{C}(19)$ & $6977(7)$ & $12070(5)$ & $4336(5)$ & $21(1)$ \\
\hline$C(16)$ & $6214(7)$ & $11453(5)$ & $9364(5)$ & $21(1)$ \\
\hline $\mathrm{C}(14)$ & $6377(8)$ & $13470(5)$ & $9286(5)$ & $25(2)$ \\
\hline $\mathrm{C}(12)$ & $9496(7)$ & $7286(5)$ & $2833(5)$ & $20(1)$ \\
\hline $\mathrm{C}(11)$ & $8626(7)$ & $6681(5)$ & $3357(5)$ & $22(1)$ \\
\hline $\mathrm{C}(28)$ & $10287(8)$ & $6660(6)$ & $2170(5)$ & $25(2)$ \\
\hline $\mathrm{C}(9)$ & $8380(7)$ & $8439(5)$ & $3907(5)$ & $18(1)$ \\
\hline$C(8)$ & $7899(7)$ & $9334(5)$ & $4399(5)$ & $20(1)$ \\
\hline $\mathrm{C}(24)$ & $9101(7)$ & $11904(5)$ & $3468(5)$ & $24(1)$ \\
\hline $\mathrm{C}(30)$ & 11106(7) & $5967(5)$ & 2891(6) & $25(2)$ \\
\hline $\mathrm{C}(17)$ & $7050(8)$ & $12574(6)$ & $9828(5)$ & $24(2)$ \\
\hline $\mathrm{C}(21)$ & $8496(7)$ & $12615(5)$ & $4237(6)$ & $25(2)$ \\
\hline$C(22)$ & $8059(7)$ & $10836(5)$ & $3102(5)$ & $20(1)$ \\
\hline $\mathrm{C}(29)$ & $9149(9)$ & $5895(7)$ & $1374(6)$ & $35(2)$ \\
\hline $\mathrm{C}(18)$ & $4659(7)$ & $11429(6)$ & $9522(5)$ & $25(2)$ \\
\hline$C(20)$ & $6026(7)$ & $11892(6)$ & $3249(5)$ & $25(2)$ \\
\hline
\end{tabular}




$\begin{array}{lllll}\mathrm{C}(5) & 6876(7) & 10531(5) & 5605(5) & 18(1) \\ \mathrm{C}(25) & 8547(8) & 5478(5) & 3117(6) & 27(2) \\ \mathrm{C}(15) & 3964(7) & 12292(6) & 8935(5) & 27(2) \\ \mathrm{C}(26) & 8091(8) & 5229(6) & 1918(6) & 34(2) \\ \mathrm{C}(23) & 6636(7) & 11120(6) & 2528(5) & 23(1) \\ \mathrm{C}(27) & 10083(7) & 5234(5) & 3410(5) & 24(1) \\ \mathrm{C}(31) & 7291(10) & 6901(7) & 8038(7) & 48(2)\end{array}$

Table S6. Bond lengths $[\AA]$ and angles $\left[{ }^{\circ}\right]$ for $\mathbf{1}(3 \mathrm{~T})^{++} \mathrm{SbF}_{6}^{-} \mathrm{CH}_{2} \mathrm{Cl}_{2}$.

\begin{tabular}{ll}
\hline $\mathrm{S}(1)-\mathrm{C}(1)$ & $1.695(6)$ \\
$\mathrm{S}(1)-\mathrm{C}(4)$ & $1.762(6)$ \\
$\mathrm{C}(3)-\mathrm{C}(4)$ & $1.374(13)$ \\
$\mathrm{C}(3)-\mathrm{C}(2)$ & $1.404(14)$ \\
$\mathrm{S}(11)-\mathrm{C}(2)$ & $1.711(14)$ \\
$\mathrm{S}(11)-\mathrm{C}(4)$ & $1.775(13)$ \\
$\mathrm{C}(53)-\mathrm{C}(4)$ & $1.372(19)$ \\
$\mathrm{C}(53)-\mathrm{C}(1)$ & $1.42(2)$ \\
$\mathrm{S}(3)-\mathrm{C}(12)$ & $1.705(6)$ \\
$\mathrm{S}(3)-\mathrm{C}(9)$ & $1.755(6)$ \\
$\mathrm{C}(10)-\mathrm{C}(9)$ & $1.384(13)$ \\
$\mathrm{C}(10)-\mathrm{C}(11)$ & $1.410(13)$ \\
$\mathrm{S}(33)-\mathrm{C}(11)$ & $1.691(14)$ \\
$\mathrm{S}(33)-\mathrm{C}(9)$ & $1.776(13)$ \\
$\mathrm{C}(60)-\mathrm{C}(9)$ & $1.39(2)$ \\
$\mathrm{C}(60)-\mathrm{C}(12)$ & $1.43(2)$ \\
$\mathrm{Sb}(2)-\mathrm{F}(5)$ & $1.873(4)$ \\
$\mathrm{Sb}(2)-\mathrm{F}(5) \# 1$ & $1.873(4)$ \\
$\mathrm{Sb}(2)-\mathrm{F}(6) \# 1$ & $1.883(4)$ \\
$\mathrm{Sb}(2)-\mathrm{F}(6)$ & $1.883(4)$ \\
& \\
&
\end{tabular}




\begin{tabular}{|c|c|}
\hline $\mathrm{Sb}(2)-\mathrm{F}(4) \# 1$ & $1.884(4)$ \\
\hline $\mathrm{Sb}(2)-\mathrm{F}(4)$ & $1.884(4)$ \\
\hline $\mathrm{Sb}(1)-\mathrm{F}(2) \# 2$ & $1.877(4)$ \\
\hline $\mathrm{Sb}(1)-\mathrm{F}(2)$ & $1.877(4)$ \\
\hline $\mathrm{Sb}(1)-\mathrm{F}(3) \# 2$ & $1.885(4)$ \\
\hline $\mathrm{Sb}(1)-\mathrm{F}(3)$ & $1.885(4)$ \\
\hline $\mathrm{Sb}(1)-\mathrm{F}(1) \# 2$ & $1.891(5)$ \\
\hline $\mathrm{Sb}(1)-\mathrm{F}(1)$ & $1.891(5)$ \\
\hline$S(2)-C(8)$ & $1.747(6)$ \\
\hline$S(2)-C(5)$ & $1.751(6)$ \\
\hline $\mathrm{Cl}(2)-\mathrm{C}(31)$ & $1.766(10)$ \\
\hline $\mathrm{Cl}(1)-\mathrm{C}(31)$ & $1.757(10)$ \\
\hline$C(2)-C(1)$ & $1.394(9)$ \\
\hline $\mathrm{C}(2)-\mathrm{C}(16)$ & $1.519(9)$ \\
\hline$C(6)-C(7)$ & $1.388(9)$ \\
\hline$C(6)-C(5)$ & $1.425(9)$ \\
\hline $\mathrm{C}(6)-\mathrm{C}(19)$ & $1.504(9)$ \\
\hline $\mathrm{C}(1)-\mathrm{C}(13)$ & $1.496(10)$ \\
\hline$C(13)-C(15)$ & $1.559(10)$ \\
\hline$C(13)-C(14)$ & $1.581(10)$ \\
\hline$C(4)-C(5)$ & $1.421(9)$ \\
\hline $\mathrm{C}(7)-\mathrm{C}(8)$ & $1.419(9)$ \\
\hline$C(7)-C(22)$ & $1.506(9)$ \\
\hline $\mathrm{C}(19)-\mathrm{C}(20)$ & $1.547(9)$ \\
\hline $\mathrm{C}(19)-\mathrm{C}(21)$ & $1.563(9)$ \\
\hline$C(16)-C(18)$ & $1.548(9)$ \\
\hline$C(16)-C(17)$ & $1.555(9)$ \\
\hline $\mathrm{C}(14)-\mathrm{C}(17)$ & $1.550(9)$ \\
\hline $\mathrm{C}(12)-\mathrm{C}(11)$ & $1.372(10)$ \\
\hline $\mathrm{C}(12)-\mathrm{C}(28)$ & $1.509(9)$ \\
\hline $\mathrm{C}(11)-\mathrm{C}(25)$ & $1.522(9)$ \\
\hline $\mathrm{C}(28)-\mathrm{C}(30)$ & $1.541(10)$ \\
\hline
\end{tabular}




\begin{tabular}{|c|c|}
\hline $\mathrm{C}(28)-\mathrm{C}(29)$ & $1.547(11)$ \\
\hline $\mathrm{C}(9)-\mathrm{C}(8)$ & $1.437(9)$ \\
\hline $\mathrm{C}(24)-\mathrm{C}(21)$ & $1.547(9)$ \\
\hline $\mathrm{C}(24)-\mathrm{C}(22)$ & $1.558(9)$ \\
\hline $\mathrm{C}(30)-\mathrm{C}(27)$ & $1.548(10)$ \\
\hline $\mathrm{C}(22)-\mathrm{C}(23)$ & $1.554(9)$ \\
\hline$C(29)-C(26)$ & $1.546(12)$ \\
\hline $\mathrm{C}(18)-\mathrm{C}(15)$ & $1.553(10)$ \\
\hline$C(20)-C(23)$ & $1.555(9)$ \\
\hline $\mathrm{C}(25)-\mathrm{C}(27)$ & $1.547(10)$ \\
\hline$C(25)-C(26)$ & $1.565(11)$ \\
\hline$C(1)-S(1)-C(4)$ & $90.2(3)$ \\
\hline $\mathrm{C}(4)-\mathrm{C}(3)-\mathrm{C}(2)$ & 115.2(8) \\
\hline$C(2)-S(11)-C(4)$ & $84.6(7)$ \\
\hline $\mathrm{C}(4)-\mathrm{C}(53)-\mathrm{C}(1)$ & $123.0(16)$ \\
\hline $\mathrm{C}(12)-\mathrm{S}(3)-\mathrm{C}(9)$ & $89.7(3)$ \\
\hline $\mathrm{C}(9)-\mathrm{C}(10)-\mathrm{C}(11)$ & $114.3(8)$ \\
\hline $\mathrm{C}(11)-\mathrm{S}(33)-\mathrm{C}(9)$ & $85.1(7)$ \\
\hline$C(9)-C(60)-C(12)$ & $120.0(15)$ \\
\hline $\mathrm{F}(5)-\mathrm{Sb}(2)-\mathrm{F}(5) \# 1$ & $180.000(1)$ \\
\hline $\mathrm{F}(5)-\mathrm{Sb}(2)-\mathrm{F}(6) \# 1$ & $89.7(2)$ \\
\hline $\mathrm{F}(5) \# 1-\mathrm{Sb}(2)-\mathrm{F}(6) \# 1$ & $90.31(19)$ \\
\hline $\mathrm{F}(5)-\mathrm{Sb}(2)-\mathrm{F}(6)$ & $90.31(19)$ \\
\hline $\mathrm{F}(5) \# 1-\mathrm{Sb}(2)-\mathrm{F}(6)$ & $89.7(2)$ \\
\hline $\mathrm{F}(6) \# 1-\mathrm{Sb}(2)-\mathrm{F}(6)$ & $180.000(1)$ \\
\hline $\mathrm{F}(5)-\mathrm{Sb}(2)-\mathrm{F}(4) \# 1$ & $90.2(2)$ \\
\hline $\mathrm{F}(5) \# 1-\mathrm{Sb}(2)-\mathrm{F}(4) \# 1$ & $89.8(2)$ \\
\hline $\mathrm{F}(6) \# 1-\mathrm{Sb}(2)-\mathrm{F}(4) \# 1$ & 89.11(19) \\
\hline $\mathrm{F}(6)-\mathrm{Sb}(2)-\mathrm{F}(4) \# 1$ & $90.89(19)$ \\
\hline $\mathrm{F}(5)-\mathrm{Sb}(2)-\mathrm{F}(4)$ & $89.8(2)$ \\
\hline $\mathrm{F}(5) \# 1-\mathrm{Sb}(2)-\mathrm{F}(4)$ & $90.2(2)$ \\
\hline
\end{tabular}




\begin{tabular}{|c|c|}
\hline $\mathrm{F}(6) \# 1-\mathrm{Sb}(2)-\mathrm{F}(4)$ & $90.89(19)$ \\
\hline $\mathrm{F}(6)-\mathrm{Sb}(2)-\mathrm{F}(4)$ & $89.11(19)$ \\
\hline $\mathrm{F}(4) \# 1-\mathrm{Sb}(2)-\mathrm{F}(4)$ & $180.00(13)$ \\
\hline $\mathrm{F}(2) \# 2-\mathrm{Sb}(1)-\mathrm{F}(2)$ & $180.000(2)$ \\
\hline $\mathrm{F}(2) \# 2-\mathrm{Sb}(1)-\mathrm{F}(3) \# 2$ & $88.6(2)$ \\
\hline $\mathrm{F}(2)-\mathrm{Sb}(1)-\mathrm{F}(3) \# 2$ & $91.4(2)$ \\
\hline $\mathrm{F}(2) \# 2-\mathrm{Sb}(1)-\mathrm{F}(3)$ & $91.4(2)$ \\
\hline $\mathrm{F}(2)-\mathrm{Sb}(1)-\mathrm{F}(3)$ & $88.6(2)$ \\
\hline $\mathrm{F}(3) \# 2-\mathrm{Sb}(1)-\mathrm{F}(3)$ & $180.000(2)$ \\
\hline $\mathrm{F}(2) \# 2-\mathrm{Sb}(1)-\mathrm{F}(1) \# 2$ & $89.9(2)$ \\
\hline $\mathrm{F}(2)-\mathrm{Sb}(1)-\mathrm{F}(1) \# 2$ & $90.1(2)$ \\
\hline $\mathrm{F}(3) \# 2-\mathrm{Sb}(1)-\mathrm{F}(1) \# 2$ & $90.3(2)$ \\
\hline $\mathrm{F}(3)-\mathrm{Sb}(1)-\mathrm{F}(1) \# 2$ & $89.7(2)$ \\
\hline $\mathrm{F}(2) \# 2-\mathrm{Sb}(1)-\mathrm{F}(1)$ & $90.1(2)$ \\
\hline $\mathrm{F}(2)-\mathrm{Sb}(1)-\mathrm{F}(1)$ & $89.9(2)$ \\
\hline $\mathrm{F}(3) \# 2-\mathrm{Sb}(1)-\mathrm{F}(1)$ & $89.7(2)$ \\
\hline $\mathrm{F}(3)-\mathrm{Sb}(1)-\mathrm{F}(1)$ & $90.3(2)$ \\
\hline $\mathrm{F}(1) \# 2-\mathrm{Sb}(1)-\mathrm{F}(1)$ & $180.000(1)$ \\
\hline$C(8)-S(2)-C(5)$ & $92.7(3)$ \\
\hline $\mathrm{C}(1)-\mathrm{C}(2)-\mathrm{C}(3)$ & $109.3(7)$ \\
\hline $\mathrm{C}(1)-\mathrm{C}(2)-\mathrm{C}(16)$ & $113.2(5)$ \\
\hline$C(3)-C(2)-C(16)$ & $137.6(7)$ \\
\hline$C(1)-C(2)-S(11)$ & $126.1(7)$ \\
\hline $\mathrm{C}(3)-\mathrm{C}(2)-\mathrm{S}(11)$ & $16.9(6)$ \\
\hline$C(16)-C(2)-S(11)$ & $120.7(7)$ \\
\hline$C(7)-C(6)-C(5)$ & $113.8(5)$ \\
\hline$C(7)-C(6)-C(19)$ & $113.7(5)$ \\
\hline$C(5)-C(6)-C(19)$ & $132.3(6)$ \\
\hline$C(2)-C(1)-C(53)$ & $97.1(10)$ \\
\hline $\mathrm{C}(2)-\mathrm{C}(1)-\mathrm{C}(13)$ & $115.7(5)$ \\
\hline $\mathrm{C}(53)-\mathrm{C}(1)-\mathrm{C}(13)$ & $147.2(10)$ \\
\hline$C(2)-C(1)-S(1)$ & $115.0(5)$ \\
\hline
\end{tabular}




\begin{tabular}{|c|c|}
\hline $\mathrm{C}(53)-\mathrm{C}(1)-\mathrm{S}(1)$ & $18.0(8)$ \\
\hline $\mathrm{C}(13)-\mathrm{C}(1)-\mathrm{S}(1)$ & 129.2(5) \\
\hline$C(1)-C(13)-C(15)$ & $106.5(6)$ \\
\hline $\mathrm{C}(1)-\mathrm{C}(13)-\mathrm{C}(14)$ & $106.3(5)$ \\
\hline $\mathrm{C}(15)-\mathrm{C}(13)-\mathrm{C}(14)$ & $107.5(5)$ \\
\hline $\mathrm{C}(53)-\mathrm{C}(4)-\mathrm{C}(3)$ & $95.5(13)$ \\
\hline$C(53)-C(4)-C(5)$ & $135.9(11)$ \\
\hline$C(3)-C(4)-C(5)$ & $128.6(6)$ \\
\hline$C(53)-C(4)-S(1)$ & $14.8(10)$ \\
\hline $\mathrm{C}(3)-\mathrm{C}(4)-\mathrm{S}(1)$ & $110.3(6)$ \\
\hline$C(5)-C(4)-S(1)$ & $121.1(4)$ \\
\hline$C(53)-C(4)-S(11)$ & $109.2(10)$ \\
\hline $\mathrm{C}(3)-\mathrm{C}(4)-\mathrm{S}(11)$ & $13.8(7)$ \\
\hline$C(5)-C(4)-S(11)$ & $114.9(6)$ \\
\hline$S(1)-C(4)-S(11)$ & $124.0(6)$ \\
\hline $\mathrm{C}(6)-\mathrm{C}(7)-\mathrm{C}(8)$ & $113.5(5)$ \\
\hline$C(6)-C(7)-C(22)$ & $114.4(5)$ \\
\hline $\mathrm{C}(8)-\mathrm{C}(7)-\mathrm{C}(22)$ & $132.0(6)$ \\
\hline$C(6)-C(19)-C(20)$ & $108.2(5)$ \\
\hline$C(6)-C(19)-C(21)$ & $106.6(5)$ \\
\hline $\mathrm{C}(20)-\mathrm{C}(19)-\mathrm{C}(21)$ & $108.2(5)$ \\
\hline $\mathrm{C}(2)-\mathrm{C}(16)-\mathrm{C}(18)$ & $108.5(5)$ \\
\hline$C(2)-C(16)-C(17)$ & $106.2(5)$ \\
\hline $\mathrm{C}(18)-\mathrm{C}(16)-\mathrm{C}(17)$ & $107.3(5)$ \\
\hline $\mathrm{C}(17)-\mathrm{C}(14)-\mathrm{C}(13)$ & $110.1(5)$ \\
\hline $\mathrm{C}(11)-\mathrm{C}(12)-\mathrm{C}(60)$ & $98.6(10)$ \\
\hline $\mathrm{C}(11)-\mathrm{C}(12)-\mathrm{C}(28)$ & $115.2(5)$ \\
\hline $\mathrm{C}(60)-\mathrm{C}(12)-\mathrm{C}(28)$ & $145.3(10)$ \\
\hline $\mathrm{C}(11)-\mathrm{C}(12)-\mathrm{S}(3)$ & $115.6(5)$ \\
\hline$C(60)-C(12)-S(3)$ & $18.3(13)$ \\
\hline $\mathrm{C}(28)-\mathrm{C}(12)-\mathrm{S}(3)$ & $129.2(5)$ \\
\hline$C(12)-C(11)-C(10)$ & $109.7(7)$ \\
\hline
\end{tabular}




\begin{tabular}{|c|c|}
\hline$C(12)-C(11)-C(25)$ & $114.2(6)$ \\
\hline$C(10)-C(11)-C(25)$ & $136.2(7)$ \\
\hline$C(12)-C(11)-S(33)$ & $125.9(7)$ \\
\hline $\mathrm{C}(10)-\mathrm{C}(11)-\mathrm{S}(33)$ & $16.5(6)$ \\
\hline$C(25)-C(11)-S(33)$ & $119.8(7)$ \\
\hline$C(12)-C(28)-C(30)$ & $106.0(5)$ \\
\hline $\mathrm{C}(12)-\mathrm{C}(28)-\mathrm{C}(29)$ & $106.8(6)$ \\
\hline $\mathrm{C}(30)-\mathrm{C}(28)-\mathrm{C}(29)$ & $107.7(6)$ \\
\hline$C(10)-C(9)-C(60)$ & $96.2(13)$ \\
\hline $\mathrm{C}(10)-\mathrm{C}(9)-\mathrm{C}(8)$ & $128.7(6)$ \\
\hline $\mathrm{C}(60)-\mathrm{C}(9)-\mathrm{C}(8)$ & $135.0(11)$ \\
\hline $\mathrm{C}(10)-\mathrm{C}(9)-\mathrm{S}(3)$ & $110.8(6)$ \\
\hline $\mathrm{C}(60)-\mathrm{C}(9)-\mathrm{S}(3)$ & $16.1(16)$ \\
\hline $\mathrm{C}(8)-\mathrm{C}(9)-\mathrm{S}(3)$ & $120.5(5)$ \\
\hline$C(10)-C(9)-S(33)$ & $12.8(7)$ \\
\hline$C(60)-C(9)-S(33)$ & $108.4(11)$ \\
\hline $\mathrm{C}(8)-\mathrm{C}(9)-\mathrm{S}(33)$ & 116.1(6) \\
\hline$S(3)-C(9)-S(33)$ & $123.4(6)$ \\
\hline $\mathrm{C}(7)-\mathrm{C}(8)-\mathrm{C}(9)$ & $129.4(6)$ \\
\hline$C(7)-C(8)-S(2)$ & $110.2(5)$ \\
\hline $\mathrm{C}(9)-\mathrm{C}(8)-\mathrm{S}(2)$ & $120.3(5)$ \\
\hline$C(21)-C(24)-C(22)$ & $110.3(5)$ \\
\hline$C(28)-C(30)-C(27)$ & $111.2(6)$ \\
\hline$C(14)-C(17)-C(16)$ & $110.6(6)$ \\
\hline$C(24)-C(21)-C(19)$ & $109.7(5)$ \\
\hline$C(7)-C(22)-C(23)$ & $106.6(5)$ \\
\hline $\mathrm{C}(7)-\mathrm{C}(22)-\mathrm{C}(24)$ & $107.5(5)$ \\
\hline$C(23)-C(22)-C(24)$ & $107.6(5)$ \\
\hline$C(26)-C(29)-C(28)$ & $110.8(6)$ \\
\hline $\mathrm{C}(16)-\mathrm{C}(18)-\mathrm{C}(15)$ & $110.5(6)$ \\
\hline$C(19)-C(20)-C(23)$ & $109.7(5)$ \\
\hline$C(4)-C(5)-C(6)$ & $129.6(6)$ \\
\hline
\end{tabular}




$\begin{array}{ll}\mathrm{C}(4)-\mathrm{C}(5)-\mathrm{S}(2) & 120.4(5) \\ \mathrm{C}(6)-\mathrm{C}(5)-\mathrm{S}(2) & 109.7(5) \\ \mathrm{C}(11)-\mathrm{C}(25)-\mathrm{C}(27) & 106.7(6) \\ \mathrm{C}(11)-\mathrm{C}(25)-\mathrm{C}(26) & 106.4(6) \\ \mathrm{C}(27)-\mathrm{C}(25)-\mathrm{C}(26) & 107.1(6) \\ \mathrm{C}(18)-\mathrm{C}(15)-\mathrm{C}(13) & 110.5(5) \\ \mathrm{C}(29)-\mathrm{C}(26)-\mathrm{C}(25) & 110.4(6) \\ \mathrm{C}(22)-\mathrm{C}(23)-\mathrm{C}(20) & 110.3(5) \\ \mathrm{C}(25)-\mathrm{C}(27)-\mathrm{C}(30) & 110.1(5) \\ \mathrm{Cl}(1)-\mathrm{C}(31)-\mathrm{Cl}(2) & 113.2(5)\end{array}$

Symmetry transformations used to generate equivalent atoms:

$\# 1-x+1,-y+1,-z+1 \quad \# 2-x+2,-y+2,-z+2$

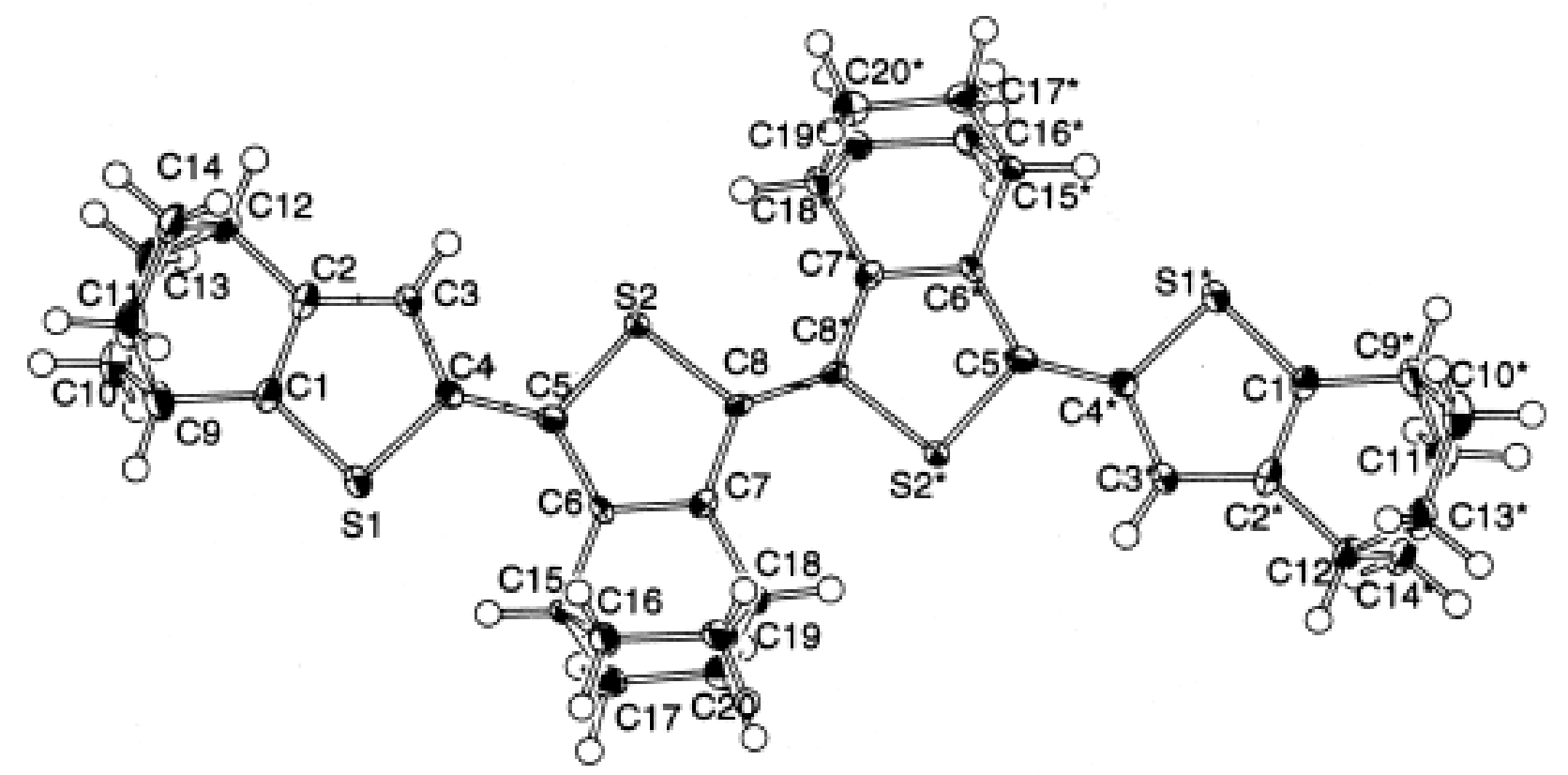

Figure S3. ORTEP drawing (50\% probability) showing the X-ray structure of $\mathbf{1}(4 \mathrm{~T})^{2+}\left(\mathrm{SbF}_{6}^{-}\right)_{2}$.

Table S7. Crystal data and structure refinement for $\mathbf{1}(4 \mathrm{~T})^{2+}\left(\mathrm{SbF}_{6}^{-}\right)_{2}$. 
Identification code

Empirical formula

Formula weight

Temperature

Wavelength

Crystal system

Space group

Unit cell dimensions

Volume

Z

Density (calculated)

Absorption coefficient

$\mathrm{F}(000)$

Crystal size

Theta range for data collection

Index ranges

Reflections collected

Independent reflections

Completeness to theta $=25.00^{\circ}$

Max. and min. transmission

Refinement method

Data / restraints / parameters

Goodness-of-fit on F2

Final $\mathrm{R}$ indices [I $>2 \operatorname{sigma}(\mathrm{I})]$

$\mathrm{R}$ indices (all data)

Largest diff. peak and hole
4 merdc

C20 H21 F6 S2 Sb

561.24

100(2) K

$0.71069 \AA$

Triclinic

P-1

$$
\begin{array}{ll}
\mathrm{a}=7.918(5) \AA & \alpha=109.542(5)^{\circ} . \\
\mathrm{b}=9.912(5) \AA & \beta=100.177(5)^{\circ} . \\
\mathrm{c}=13.964(5) \AA & \gamma=97.274(5)^{\circ} .
\end{array}
$$

996.0(9) $\AA^{3}$

2

$1.871 \mathrm{Mg} / \mathrm{m}^{3}$

$1.654 \mathrm{~mm}^{-1}$

556

$0.10 \times 0.05 \times 0.01 \mathrm{~mm}^{3}$

2.21 to $25.00^{\circ}$.

$-8<=\mathrm{h}<=9,-11<=\mathrm{k}<=10,-13<=\mathrm{l}<=16$

5208

$3453[\mathrm{R}($ int $)=0.0428]$

$98.6 \%$

0.9837 and 0.8521

Full-matrix least-squares on $\mathrm{F}^{2}$

3453 / 36 / 299

1.000

$$
\begin{aligned}
& \mathrm{R} 1=0.0509, \mathrm{wR} 2=0.0810 \\
& \mathrm{R} 1=0.0834, \mathrm{wR} 2=0.0872 \\
& 0.727 \text { and }-0.817 \mathrm{e} . \AA^{-3}
\end{aligned}
$$

Table S8. Atomic coordinates $\left(\mathrm{x} 10^{4}\right)$ and equivalent isotropic displacement parameters $\left(\AA^{2} \times 10^{3}\right)$ 
for $1(4 \mathrm{~T})^{2+}\left(\mathrm{SbF}_{6}^{-}\right)_{2}$. U(eq) is defined as one third of the trace of the orthogonalized Uij tensor.

\begin{tabular}{|c|c|c|c|c|}
\hline & $\mathrm{x}$ & $\mathrm{y}$ & $\mathrm{z}$ & $\mathrm{U}(\mathrm{eq})$ \\
\hline $\mathrm{F}(6)$ & $5721(13)$ & $4908(11)$ & 1192(6) & $62(3)$ \\
\hline $\mathrm{F}(3)$ & $6307(11)$ & $5450(8)$ & $3986(5)$ & $49(3)$ \\
\hline $\mathrm{F}(2)$ & $3666(9)$ & $4603(8)$ & $2394(7)$ & $51(2)$ \\
\hline $\mathrm{F}(5)$ & $8406(9)$ & $5744(9)$ & 2795(9) & $73(3)$ \\
\hline $\mathrm{F}(14)$ & $7900(30)$ & $6460(20)$ & $3790(13)$ & $86(9)$ \\
\hline $\mathrm{F}(11)$ & $4780(40)$ & $4950(20)$ & $3360(30)$ & $115(13)$ \\
\hline $\mathrm{F}(12)$ & $4710(40)$ & $4280(20)$ & $1400(20)$ & $110(12)$ \\
\hline$F(13)$ & $7840(30)$ & $5743(18)$ & $1860(20)$ & $81(9)$ \\
\hline $\mathrm{Sb}(1)$ & $6118(1)$ & $5230(1)$ & 2591(1) & $30(1)$ \\
\hline$S(2)$ & $11115(2)$ & $3633(2)$ & $3792(1)$ & $21(1)$ \\
\hline $\mathrm{S}(1)$ & $9424(2)$ & $-1036(2)$ & $2145(1)$ & $23(1)$ \\
\hline $\mathrm{F}(1)$ & $5713(6)$ & $7091(4)$ & $2832(4)$ & $55(1)$ \\
\hline$C(2)$ & $11958(8)$ & $-316(7)$ & $1382(5)$ & $21(2)$ \\
\hline $\mathrm{F}(4)$ & $6527(9)$ & $3371(6)$ & $2380(4)$ & $105(2)$ \\
\hline $\mathrm{C}(18)$ & $7199(7)$ & $3008(6)$ & $5225(5)$ & $20(2)$ \\
\hline $\mathrm{C}(4)$ & $10527(7)$ & $780(6)$ & $2650(5)$ & $14(1)$ \\
\hline$C(3)$ & $11855(7)$ & $967(6)$ & $2149(4)$ & $17(1)$ \\
\hline$C(6)$ & $8748(7)$ & $1777(6)$ & 3992(4) & $17(1)$ \\
\hline $\mathrm{C}(8)$ & $9873(7)$ & $4265(6)$ & $4691(5)$ & $17(2)$ \\
\hline $\mathrm{C}(20)$ & $7585(9)$ & $2015(7)$ & $5834(5)$ & $28(2)$ \\
\hline$C(15)$ & $7434(8)$ & $514(6)$ & $3941(5)$ & $21(2)$ \\
\hline $\mathrm{C}(7)$ & $8619(8)$ & $3092(6)$ & $4668(5)$ & $17(1)$ \\
\hline$C(16)$ & $5660(8)$ & $862(6)$ & $3612(5)$ & $27(2)$ \\
\hline C(19) & $5494(8)$ & $2324(6)$ & $4392(5)$ & $26(2)$ \\
\hline $\mathrm{C}(5)$ & $10048(7)$ & $1841(6)$ & $3429(5)$ & $16(1)$ \\
\hline$C(14)$ & $11882(8)$ & $-1326(7)$ & $-449(5)$ & $25(2)$ \\
\hline $\mathrm{C}(9)$ & $10742(8)$ & $-2888(6)$ & 491(5) & $26(2)$ \\
\hline$C(13)$ & $13978(8)$ & $-1941(7)$ & $814(5)$ & $31(2)$ \\
\hline
\end{tabular}




$\begin{array}{lllll}\mathrm{C}(1) & 10717(8) & -1465(6) & 1313(5) & 21(2) \\ \mathrm{C}(17) & 7636(9) & 503(7) & 5051(5) & 32(2) \\ \mathrm{C}(12) & 13096(8) & -715(6) & 634(5) & 23(2) \\ \mathrm{C}(10) & 12596(8) & -3180(7) & 766(6) & 32(2) \\ \mathrm{C}(11) & 10505(9) & -2619(7) & -549(5) & 30(2)\end{array}$

Table S9. Bond lengths $[\AA]$ and angles $\left[{ }^{\circ}\right]$ for $\mathbf{1}(4 \mathrm{~T})^{2+}\left(\mathrm{SbF}_{6}^{-}\right)_{2}$.

\begin{tabular}{ll}
\hline $\mathrm{F}(6)-\mathrm{Sb}(1)$ & $1.832(8)$ \\
$\mathrm{F}(3)-\mathrm{Sb}(1)$ & $1.863(6)$ \\
$\mathrm{F}(2)-\mathrm{Sb}(1)$ & $1.903(6)$ \\
$\mathrm{F}(5)-\mathrm{Sb}(1)$ & $1.764(7)$ \\
$\mathrm{F}(14)-\mathrm{Sb}(1)$ & $1.909(16)$ \\
$\mathrm{F}(11)-\mathrm{Sb}(1)$ & $1.694(16)$ \\
$\mathrm{F}(12)-\mathrm{Sb}(1)$ & $1.72(2)$ \\
$\mathrm{F}(13)-\mathrm{Sb}(1)$ & $1.958(18)$ \\
$\mathrm{Sb}(1)-\mathrm{F}(1)$ & $1.841(4)$ \\
$\mathrm{Sb}(1)-\mathrm{F}(4)$ & $1.847(4)$ \\
$\mathrm{S}(2)-\mathrm{C}(5)$ & $1.730(6)$ \\
$\mathrm{S}(2)-\mathrm{C}(8)$ & $1.733(6)$ \\
$\mathrm{S}(1)-\mathrm{C}(1)$ & $1.668(6)$ \\
$\mathrm{S}(1)-\mathrm{C}(4)$ & $1.743(6)$ \\
$\mathrm{C}(2)-\mathrm{C}(1)$ & $1.372(8)$ \\
$\mathrm{C}(2)-\mathrm{C}(3)$ & $1.385(8)$ \\
$\mathrm{C}(2)-\mathrm{C}(12)$ & $1.485(8)$ \\
$\mathrm{C}(18)-\mathrm{C}(7)$ & $1.485(8)$ \\
$\mathrm{C}(18)-\mathrm{C}(19)$ & $1.523(8)$ \\
$\mathrm{C}(18)-\mathrm{C}(20)$ & $1.527(9)$ \\
$\mathrm{C}(4)-\mathrm{C}(5)$ & $1.383(7)$ \\
$\mathrm{C}(4)-\mathrm{C}(3)$ & $1.388(8)$ \\
&
\end{tabular}




\begin{tabular}{|c|c|}
\hline$C(6)-C(7)$ & $1.358(7)$ \\
\hline$C(6)-C(5)$ & $1.408(8)$ \\
\hline$C(6)-C(15)$ & $1.497(8)$ \\
\hline $\mathrm{C}(8)-\mathrm{C}(8) \# 1$ & $1.390(11)$ \\
\hline $\mathrm{C}(8)-\mathrm{C}(7)$ & $1.417(8)$ \\
\hline$C(20)-C(17)$ & $1.542(8)$ \\
\hline$C(15)-C(16)$ & $1.518(8)$ \\
\hline $\mathrm{C}(15)-\mathrm{C}(17)$ & $1.532(9)$ \\
\hline$C(16)-C(19)$ & $1.532(8)$ \\
\hline $\mathrm{C}(14)-\mathrm{C}(12)$ & $1.518(8)$ \\
\hline $\mathrm{C}(14)-\mathrm{C}(11)$ & $1.524(9)$ \\
\hline $\mathrm{C}(9)-\mathrm{C}(1)$ & $1.496(8)$ \\
\hline $\mathrm{C}(9)-\mathrm{C}(10)$ & $1.539(9)$ \\
\hline $\mathrm{C}(9)-\mathrm{C}(11)$ & $1.547(9)$ \\
\hline$C(13)-C(10)$ & $1.513(9)$ \\
\hline $\mathrm{C}(13)-\mathrm{C}(12)$ & $1.545(9)$ \\
\hline $\mathrm{F}(11)-\mathrm{Sb}(1)-\mathrm{F}(12)$ & $98.4(16)$ \\
\hline $\mathrm{F}(11)-\mathrm{Sb}(1)-\mathrm{F}(5)$ & $133.9(13)$ \\
\hline $\mathrm{F}(12)-\mathrm{Sb}(1)-\mathrm{F}(5)$ & $124.8(12)$ \\
\hline $\mathrm{F}(11)-\mathrm{Sb}(1)-\mathrm{F}(6)$ & $132.5(13)$ \\
\hline $\mathrm{F}(12)-\mathrm{Sb}(1)-\mathrm{F}(6)$ & $35.6(11)$ \\
\hline $\mathrm{F}(5)-\mathrm{Sb}(1)-\mathrm{F}(6)$ & $93.3(4)$ \\
\hline $\mathrm{F}(11)-\mathrm{Sb}(1)-\mathrm{F}(1)$ & $91.5(6)$ \\
\hline $\mathrm{F}(12)-\mathrm{Sb}(1)-\mathrm{F}(1)$ & $101.3(8)$ \\
\hline $\mathrm{F}(5)-\mathrm{Sb}(1)-\mathrm{F}(1)$ & $94.8(3)$ \\
\hline $\mathrm{F}(6)-\mathrm{Sb}(1)-\mathrm{F}(1)$ & $88.9(3)$ \\
\hline $\mathrm{F}(11)-\mathrm{Sb}(1)-\mathrm{F}(4)$ & $87.6(6)$ \\
\hline $\mathrm{F}(12)-\mathrm{Sb}(1)-\mathrm{F}(4)$ & $79.7(8)$ \\
\hline $\mathrm{F}(5)-\mathrm{Sb}(1)-\mathrm{F}(4)$ & $85.2(3)$ \\
\hline $\mathrm{F}(6)-\mathrm{Sb}(1)-\mathrm{F}(4)$ & $92.3(3)$ \\
\hline $\mathrm{F}(1)-\mathrm{Sb}(1)-\mathrm{F}(4)$ & $178.8(2)$ \\
\hline
\end{tabular}




\begin{tabular}{|c|c|}
\hline $\mathrm{F}(11)-\mathrm{Sb}(1)-\mathrm{F}(3)$ & $42.1(12)$ \\
\hline $\mathrm{F}(12)-\mathrm{Sb}(1)-\mathrm{F}(3)$ & $138.9(13)$ \\
\hline $\mathrm{F}(5)-\mathrm{Sb}(1)-\mathrm{F}(3)$ & $91.9(4)$ \\
\hline $\mathrm{F}(6)-\mathrm{Sb}(1)-\mathrm{F}(3)$ & $174.5(5)$ \\
\hline $\mathrm{F}(1)-\mathrm{Sb}(1)-\mathrm{F}(3)$ & $92.4(3)$ \\
\hline $\mathrm{F}(4)-\mathrm{Sb}(1)-\mathrm{F}(3)$ & $86.4(3)$ \\
\hline $\mathrm{F}(11)-\mathrm{Sb}(1)-\mathrm{F}(2)$ & $44.6(12)$ \\
\hline $\mathrm{F}(12)-\mathrm{Sb}(1)-\mathrm{F}(2)$ & $55.8(12)$ \\
\hline $\mathrm{F}(5)-\mathrm{Sb}(1)-\mathrm{F}(2)$ & $177.3(4)$ \\
\hline $\mathrm{F}(6)-\mathrm{Sb}(1)-\mathrm{F}(2)$ & $88.0(4)$ \\
\hline $\mathrm{F}(1)-\mathrm{Sb}(1)-\mathrm{F}(2)$ & $87.5(3)$ \\
\hline $\mathrm{F}(4)-\mathrm{Sb}(1)-\mathrm{F}(2)$ & $92.4(3)$ \\
\hline $\mathrm{F}(3)-\mathrm{Sb}(1)-\mathrm{F}(2)$ & $86.7(3)$ \\
\hline $\mathrm{F}(11)-\mathrm{Sb}(1)-\mathrm{F}(14)$ & $91.1(14)$ \\
\hline $\mathrm{F}(12)-\mathrm{Sb}(1)-\mathrm{F}(14)$ & $169.8(15)$ \\
\hline $\mathrm{F}(5)-\mathrm{Sb}(1)-\mathrm{F}(14)$ & $47.6(7)$ \\
\hline $\mathrm{F}(6)-\mathrm{Sb}(1)-\mathrm{F}(14)$ & $134.2(9)$ \\
\hline $\mathrm{F}(1)-\mathrm{Sb}(1)-\mathrm{F}(14)$ & $74.7(5)$ \\
\hline $\mathrm{F}(4)-\mathrm{Sb}(1)-\mathrm{F}(14)$ & $104.5(6)$ \\
\hline $\mathrm{F}(3)-\mathrm{Sb}(1)-\mathrm{F}(14)$ & $51.2(7)$ \\
\hline $\mathrm{F}(2)-\mathrm{Sb}(1)-\mathrm{F}(14)$ & $132.2(7)$ \\
\hline $\mathrm{F}(11)-\mathrm{Sb}(1)-\mathrm{F}(13)$ & $173.0(14)$ \\
\hline $\mathrm{F}(12)-\mathrm{Sb}(1)-\mathrm{F}(13)$ & $88.4(13)$ \\
\hline $\mathrm{F}(5)-\mathrm{Sb}(1)-\mathrm{F}(13)$ & $40.5(7)$ \\
\hline $\mathrm{F}(6)-\mathrm{Sb}(1)-\mathrm{F}(13)$ & $53.8(8)$ \\
\hline $\mathrm{F}(1)-\mathrm{Sb}(1)-\mathrm{F}(13)$ & $85.4(6)$ \\
\hline $\mathrm{F}(4)-\mathrm{Sb}(1)-\mathrm{F}(13)$ & $95.5(6)$ \\
\hline $\mathrm{F}(3)-\mathrm{Sb}(1)-\mathrm{F}(13)$ & $131.6(9)$ \\
\hline $\mathrm{F}(2)-\mathrm{Sb}(1)-\mathrm{F}(13)$ & 141.2(9) \\
\hline $\mathrm{F}(14)-\mathrm{Sb}(1)-\mathrm{F}(13)$ & $82.0(10)$ \\
\hline $\mathrm{C}(5)-\mathrm{S}(2)-\mathrm{C}(8)$ & $92.3(3)$ \\
\hline$C(1)-S(1)-C(4)$ & $90.5(3)$ \\
\hline
\end{tabular}




\begin{tabular}{|c|c|}
\hline$C(1)-C(2)-C(3)$ & $111.3(5)$ \\
\hline $\mathrm{C}(1)-\mathrm{C}(2)-\mathrm{C}(12)$ & $114.0(5)$ \\
\hline$C(3)-C(2)-C(12)$ & $134.6(6)$ \\
\hline $\mathrm{C}(7)-\mathrm{C}(18)-\mathrm{C}(19)$ & $106.9(5)$ \\
\hline $\mathrm{C}(7)-\mathrm{C}(18)-\mathrm{C}(20)$ & $107.2(5)$ \\
\hline $\mathrm{C}(19)-\mathrm{C}(18)-\mathrm{C}(20)$ & $110.1(5)$ \\
\hline$C(5)-C(4)-C(3)$ & $127.4(5)$ \\
\hline$C(5)-C(4)-S(1)$ & $121.9(4)$ \\
\hline $\mathrm{C}(3)-\mathrm{C}(4)-\mathrm{S}(1)$ & $110.7(4)$ \\
\hline$C(2)-C(3)-C(4)$ & $112.8(5)$ \\
\hline$C(7)-C(6)-C(5)$ & $114.6(5)$ \\
\hline$C(7)-C(6)-C(15)$ & $114.4(5)$ \\
\hline$C(5)-C(6)-C(15)$ & $131.0(5)$ \\
\hline $\mathrm{C}(8) \# 1-\mathrm{C}(8)-\mathrm{C}(7)$ & $127.8(7)$ \\
\hline $\mathrm{C}(8) \# 1-\mathrm{C}(8)-\mathrm{S}(2)$ & $122.0(6)$ \\
\hline$C(7)-C(8)-S(2)$ & $110.3(4)$ \\
\hline$C(18)-C(20)-C(17)$ & $108.3(5)$ \\
\hline $\mathrm{C}(6)-\mathrm{C}(15)-\mathrm{C}(16)$ & $105.2(5)$ \\
\hline$C(6)-C(15)-C(17)$ & $107.7(5)$ \\
\hline $\mathrm{C}(16)-\mathrm{C}(15)-\mathrm{C}(17)$ & $108.4(5)$ \\
\hline$C(6)-C(7)-C(8)$ & $112.9(5)$ \\
\hline$C(6)-C(7)-C(18)$ & $113.8(5)$ \\
\hline$C(8)-C(7)-C(18)$ & $133.3(5)$ \\
\hline $\mathrm{C}(15)-\mathrm{C}(16)-\mathrm{C}(19)$ & $110.2(5)$ \\
\hline$C(18)-C(19)-C(16)$ & $109.8(5)$ \\
\hline$C(4)-C(5)-C(6)$ & $132.2(5)$ \\
\hline$C(4)-C(5)-S(2)$ & $117.8(4)$ \\
\hline$C(6)-C(5)-S(2)$ & $109.8(4)$ \\
\hline $\mathrm{C}(12)-\mathrm{C}(14)-\mathrm{C}(11)$ & $110.4(5)$ \\
\hline $\mathrm{C}(1)-\mathrm{C}(9)-\mathrm{C}(10)$ & $105.8(5)$ \\
\hline $\mathrm{C}(1)-\mathrm{C}(9)-\mathrm{C}(11)$ & $106.0(5)$ \\
\hline $\mathrm{C}(10)-\mathrm{C}(9)-\mathrm{C}(11)$ & $108.4(5)$ \\
\hline
\end{tabular}




$\begin{array}{ll}\mathrm{C}(10)-\mathrm{C}(13)-\mathrm{C}(12) & 109.6(5) \\ \mathrm{C}(2)-\mathrm{C}(1)-\mathrm{C}(9) & 114.3(5) \\ \mathrm{C}(2)-\mathrm{C}(1)-\mathrm{S}(1) & 114.7(5) \\ \mathrm{C}(9)-\mathrm{C}(1)-\mathrm{S}(1) & 131.0(5) \\ \mathrm{C}(15)-\mathrm{C}(17)-\mathrm{C}(20) & 111.1(5) \\ \mathrm{C}(2)-\mathrm{C}(12)-\mathrm{C}(14) & 106.1(5) \\ \mathrm{C}(2)-\mathrm{C}(12)-\mathrm{C}(13) & 108.3(6) \\ \mathrm{C}(14)-\mathrm{C}(12)-\mathrm{C}(13) & 108.5(5) \\ \mathrm{C}(13)-\mathrm{C}(10)-\mathrm{C}(9) & 111.3(6) \\ \mathrm{C}(14)-\mathrm{C}(11)-\mathrm{C}(9) & 110.4(5)\end{array}$

Symmetry transformations used to generate equivalent atoms:

$\# 1-\mathrm{x}+2,-\mathrm{y}+1,-\mathrm{z}+1$
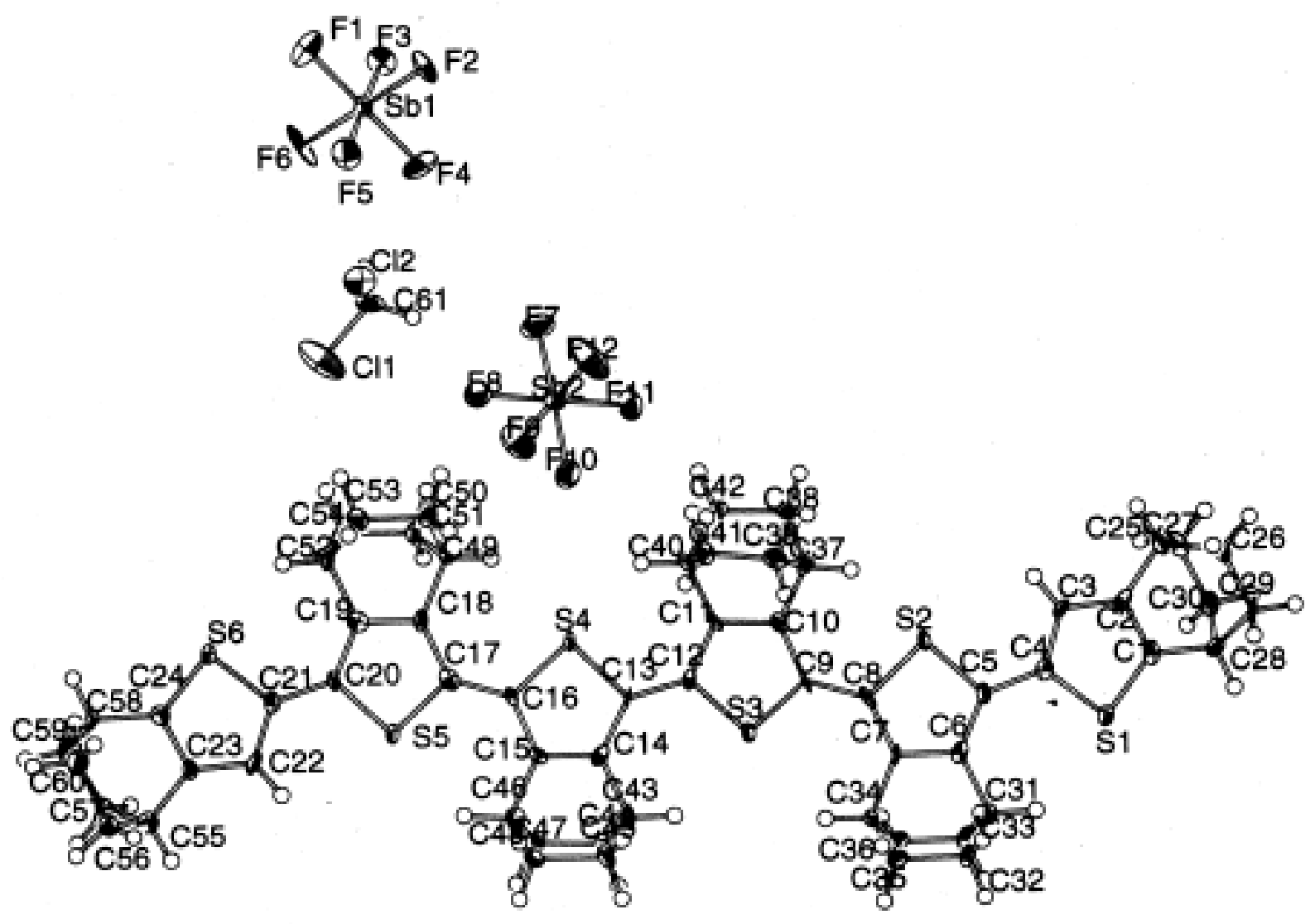

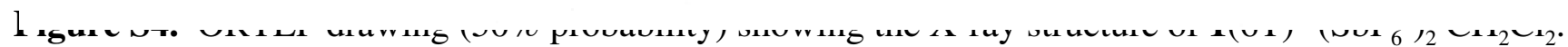

Table S10. Crystal data and structure refinement for $\mathbf{1}(6 \mathrm{~T})^{2+}\left(\mathrm{SbF}_{6}^{-}\right)_{2} \mathrm{CH}_{2} \mathrm{Cl}_{2}$. 
Empirical formula

Formula weight

Temperature

Wavelength

Crystal system

Space group

Unit cell dimensions

Volume

Z

Density (calculated)

Absorption coefficient

$\mathrm{F}(000)$

Crystal size

Theta range for data collection

Index ranges

Reflections collected

Independent reflections

Completeness to theta $=25.00^{\circ}$

Max. and min. transmission

Refinement method

Data / restraints / parameters

Goodness-of-fit on F2

Final R indices [I $>2 \operatorname{sigma}(\mathrm{I})]$

$\mathrm{R}$ indices (all data)

Largest diff. peak and hole
C61 H64 Cl2 F12 S6 Sb2

1531.88

100(2) K

$0.71069 \AA$

Triclinic

P-1

$$
\begin{array}{ll}
\mathrm{a}=11.197(5) \AA & \alpha=79.113(5)^{\circ} . \\
\mathrm{b}=16.574(5) \AA & \beta=82.739(5)^{\circ} . \\
\mathrm{c}=16.880(5) \AA & \gamma=77.222(5)^{\circ} .
\end{array}
$$

2988.3(18) $\AA^{3}$

2

$1.702 \mathrm{Mg} / \mathrm{m}^{3}$

$1.282 \mathrm{~mm}^{-1}$

1540

$0.10 \times 0.10 \times 0.02 \mathrm{~mm}^{3}$

1.87 to $25.00^{\circ}$.

$-13<=\mathrm{h}<=11,-19<=\mathrm{k}<=19,-20<=\mathrm{l}<=19$

14181

$10263[\mathrm{R}(\mathrm{int})=0.0922]$

$97.4 \%$

0.9748 and 0.8825

Full-matrix least-squares on $\mathrm{F}^{2}$

10263 / 54 / 748

0.878

$\mathrm{R} 1=0.0644, \mathrm{wR} 2=0.0993$

$\mathrm{R} 1=0.1598, \mathrm{wR} 2=0.1226$

0.822 and -0.674 e. $\AA^{-3}$

Table S11. Atomic coordinates $\left(\times 10^{4}\right)$ and equivalent isotropic displacement parameters $\left(\AA^{2} \times 10^{3}\right)$ for $\mathbf{1}(6 \mathrm{~T})^{2+}\left(\mathrm{SbF}_{6}^{-}\right)_{2} \mathrm{CH}_{2} \mathrm{Cl}_{2}$. U(eq) is defined as one third of the trace of the orthogonalized Uij tensor. 


\begin{tabular}{|c|c|c|c|c|}
\hline & $\mathrm{x}$ & $\mathrm{y}$ & $\mathrm{z}$ & $\mathrm{U}(\mathrm{eq})$ \\
\hline $\mathrm{Sb}(1)$ & $1367(1)$ & $2568(1)$ & $9310(1)$ & $23(1)$ \\
\hline $\operatorname{Sb}(2)$ & $3016(1)$ & $3393(1)$ & $4504(1)$ & $28(1)$ \\
\hline$S(4)$ & $5695(2)$ & $2502(1)$ & $1667(1)$ & $16(1)$ \\
\hline$S(2)$ & $1841(2)$ & $6244(1)$ & $-1062(1)$ & $18(1)$ \\
\hline$S(3)$ & $4507(2)$ & $3866(2)$ & $-703(1)$ & 19(1) \\
\hline$S(6)$ & $9768(2)$ & $-1346(2)$ & $4302(2)$ & $25(1)$ \\
\hline$S(5)$ & $8357(2)$ & 102(2) & 2031(1) & $22(1)$ \\
\hline $\mathrm{S}(1)$ & $-113(2)$ & $7496(2)$ & $-3197(1)$ & $22(1)$ \\
\hline $\mathrm{Cl}(2)$ & $4854(3)$ & $2295(2)$ & 7191(2) & $46(1)$ \\
\hline $\mathrm{Cl}(1)$ & $4226(3)$ & $808(2)$ & $6851(3)$ & $78(1)$ \\
\hline $\mathrm{F}(3)$ & $-277(5)$ & $2625(3)$ & $9803(3)$ & $35(2)$ \\
\hline $\mathrm{F}(8)$ & $4379(6)$ & $2845(4)$ & $5077(3)$ & $48(2)$ \\
\hline $\mathrm{F}(5)$ & $2971(5)$ & $2520(4)$ & $8792(4)$ & $46(2)$ \\
\hline C(19) & $7873(9)$ & $392(6)$ & $3506(5)$ & $17(2)$ \\
\hline$C(25)$ & $-1882(9)$ & $9133(6)$ & $-1798(6)$ & $24(2)$ \\
\hline $\mathrm{F}(10)$ & $4012(6)$ & $3313(4)$ & $3524(3)$ & $53(2)$ \\
\hline $\mathrm{F}(7)$ & $2084(6)$ & $3442(4)$ & $5487(3)$ & $57(2)$ \\
\hline $\mathrm{F}(4)$ & $763(6)$ & $2651(4)$ & $8303(3)$ & $50(2)$ \\
\hline $\mathrm{F}(2)$ & $1157(5)$ & $3740(3)$ & $9173(4)$ & $37(2)$ \\
\hline $\mathrm{F}(1)$ & $1964(6)$ & $2484(4)$ & 10311(3) & $54(2)$ \\
\hline$F(6)$ & $1564(6)$ & $1405(4)$ & $9470(4)$ & $57(2)$ \\
\hline$C(7)$ & $2828(8)$ & $5198(5)$ & $-2039(5)$ & $15(2)$ \\
\hline$C(14)$ & $6105(9)$ & $2100(6)$ & $218(5)$ & $18(2)$ \\
\hline$C(46)$ & $7405(8)$ & $712(5)$ & $267(5)$ & $18(2)$ \\
\hline$C(15)$ & $6786(8)$ & $1453(5)$ & $694(5)$ & $13(2)$ \\
\hline C(49) & $6227(8)$ & $1626(5)$ & $3539(5)$ & $16(2)$ \\
\hline$C(37)$ & 1841(9) & $5157(6)$ & $738(5)$ & $20(2)$ \\
\hline $\mathrm{F}(11)$ & $1643(6)$ & $3939(4)$ & $3943(4)$ & $51(2)$ \\
\hline$C(52)$ & $7689(9)$ & $443(6)$ & $4391(5)$ & $18(2)$ \\
\hline
\end{tabular}




\begin{tabular}{|c|c|c|c|c|}
\hline $\mathrm{C}(9)$ & $3286(9)$ & $4707(5)$ & $-554(6)$ & $18(2)$ \\
\hline $\mathrm{C}(17)$ & $7244(9)$ & $1003(5)$ & $2200(6)$ & $19(2)$ \\
\hline$C(5)$ & 1503(9) & $6516(6)$ & $-2070(5)$ & $17(2)$ \\
\hline$C(54)$ & 6371(9) & $378(6)$ & $4706(5)$ & $20(2)$ \\
\hline C(39) & 1100(9) & $4496(6)$ & $1182(5)$ & $22(2)$ \\
\hline $\mathrm{C}(11)$ & $3660(8)$ & $4037(5)$ & $790(5)$ & $12(2)$ \\
\hline$C(2)$ & $-928(9)$ & $8445(6)$ & $-2122(5)$ & $18(2)$ \\
\hline$C(43)$ & $6124(9)$ & $1985(6)$ & $-647(5)$ & $23(2)$ \\
\hline $\mathrm{F}(9)$ & $2647(6)$ & $2361(4)$ & $4471(4)$ & $59(2)$ \\
\hline $\mathrm{F}(12)$ & $3411(6)$ & $4417(4)$ & $4526(5)$ & $69(2)$ \\
\hline$C(16)$ & $6678(8)$ & $1540(5)$ & $1536(5)$ & $12(2)$ \\
\hline C(13) & $5418(8)$ & $2757(5)$ & $622(5)$ & $12(2)$ \\
\hline $\mathrm{C}(24)$ & $10650(9)$ & $-2257(6)$ & $4031(6)$ & $22(2)$ \\
\hline$C(20)$ & $8565(9)$ & $-202(6)$ & $3066(5)$ & $19(2)$ \\
\hline $\mathrm{C}(34)$ & $3368(9)$ & $4488(6)$ & $-2509(5)$ & $21(2)$ \\
\hline $\mathrm{C}(23)$ & 10649(9) & $-2309(5)$ & $3228(5)$ & $16(2)$ \\
\hline$C(10)$ & 2939(9) & $4684(5)$ & 291(5) & $17(2)$ \\
\hline$C(8)$ & $2768(8)$ & $5273(5)$ & $-1213(5)$ & $15(2)$ \\
\hline$C(47)$ & $8244(9)$ & $1050(6)$ & $-455(5)$ & $21(2)$ \\
\hline$C(56)$ & $12673(9)$ & $-3221(6)$ & $3167(6)$ & $28(3)$ \\
\hline $\mathrm{C}(40)$ & $3213(8)$ & $3981(5)$ & $1679(5)$ & $17(2)$ \\
\hline$C(53)$ & $7890(9)$ & $1302(5)$ & $4502(5)$ & $23(2)$ \\
\hline $\mathrm{C}(41)$ & 1894(9) & $3825(5)$ & $1771(5)$ & $19(2)$ \\
\hline $\mathrm{C}(31)$ & 2085(9) & $5777(6)$ & $-3384(5)$ & $20(2)$ \\
\hline$C(50)$ & 6994(9) & $2010(5)$ & $4002(5)$ & $21(2)$ \\
\hline $\mathrm{C}(21)$ & $9355(9)$ & $-994(6)$ & $3317(5)$ & $20(2)$ \\
\hline $\mathrm{C}(18)$ & $7083(8)$ & $1037(5)$ & $3044(5)$ & $17(2)$ \\
\hline$C(45)$ & 7488(9) & $1818(6)$ & $-1000(6)$ & $26(3)$ \\
\hline$C(44)$ & $5598(9)$ & $1177(5)$ & $-616(6)$ & $24(2)$ \\
\hline$C(26)$ & $-1898(9)$ & $9956(5)$ & $-2404(5)$ & $26(2)$ \\
\hline $\mathrm{C}(1)$ & $-1122(9)$ & $8338(5)$ & $-2870(5)$ & $16(2)$ \\
\hline$C(6)$ & 2119(8) & $5885(6)$ & $-2513(5)$ & $16(2)$ \\
\hline
\end{tabular}




\begin{tabular}{|c|c|c|c|c|}
\hline$C(12)$ & $4574(9)$ & $3495(5)$ & $349(5)$ & $17(2)$ \\
\hline $\mathrm{C}(22)$ & 9874(9) & $-1599(6)$ & $2822(5)$ & $21(2)$ \\
\hline $\mathrm{C}(61)$ & $3742(10)$ & $1816(6)$ & $7028(6)$ & $32(3)$ \\
\hline C(29) & $-2009(9)$ & $9830(5)$ & $-3266(5)$ & $23(2)$ \\
\hline $\mathrm{C}(30)$ & $-3341(9)$ & $8781(6)$ & $-2650(5)$ & $23(2)$ \\
\hline $\mathrm{C}(28)$ & $-2191(9)$ & $8912(6)$ & $-3259(5)$ & $22(2)$ \\
\hline $\mathrm{C}(32)$ & $3432(8)$ & $5590(6)$ & $-3764(5)$ & $22(2)$ \\
\hline$C(33)$ & $1544(9)$ & $5019(6)$ & $-3347(6)$ & $28(3)$ \\
\hline$C(3)$ & $50(8)$ & $7842(5)$ & $-1768(5)$ & $17(2)$ \\
\hline $\mathrm{C}(51)$ & $5473(9)$ & $1082(5)$ & $4183(5)$ & $18(2)$ \\
\hline$C(57)$ & 10819(10) & $-3828(6)$ & $3583(6)$ & $29(3)$ \\
\hline C(59) & $10960(10)$ & $-3775(6)$ & $4477(5)$ & $31(3)$ \\
\hline$C(4)$ & $615(8)$ & $7277(6)$ & $-2282(5)$ & $18(2)$ \\
\hline $\mathrm{C}(38)$ & 2301(10) & $5542(5)$ & $1388(5)$ & $24(3)$ \\
\hline $\mathrm{C}(35)$ & 4181(9) & $4826(6)$ & $-3252(5)$ & $24(3)$ \\
\hline$C(55)$ & 11329(9) & $-3133(6)$ & $3001(6)$ & $21(2)$ \\
\hline $\mathrm{C}(27)$ & $-3158(9)$ & $8908(6)$ & $-1803(6)$ & $29(3)$ \\
\hline$C(58)$ & 11482(9) & $-2969(6)$ & $4509(5)$ & $24(2)$ \\
\hline$C(36)$ & 2304(10) & $4251(6)$ & $-2840(6)$ & $29(3)$ \\
\hline$C(42)$ & $3175(9)$ & $4845(6)$ & $1923(5)$ & $25(2)$ \\
\hline $\mathrm{C}(48)$ & $6344(9)$ & $428(6)$ & $-45(6)$ & $24(2)$ \\
\hline$C(60)$ & $12762(9)$ & $-3074(6)$ & $4058(6)$ & $29(3)$ \\
\hline
\end{tabular}

Table S12. Bond lengths $[\AA]$ and angles $\left[^{\circ}\right]$ for $\mathbf{1}(6 \mathrm{~T})^{2+}\left(\mathrm{SbF}_{6}^{-}\right)_{2} \mathrm{CH}_{2} \mathrm{Cl}_{2}$.

\begin{tabular}{ll}
\hline $\mathrm{Sb}(1)-\mathrm{F}(6)$ & $1.861(6)$ \\
$\mathrm{Sb}(1)-\mathrm{F}(1)$ & $1.864(6)$ \\
$\mathrm{Sb}(1)-\mathrm{F}(2)$ & $1.877(5)$ \\
$\mathrm{Sb}(1)-\mathrm{F}(4)$ & $1.878(5)$ \\
$\mathrm{Sb}(1)-\mathrm{F}(5)$ & $1.888(6)$
\end{tabular}




\begin{tabular}{|c|c|}
\hline $\mathrm{Sb}(1)-\mathrm{F}(3)$ & $1.912(5)$ \\
\hline $\mathrm{Sb}(2)-\mathrm{F}(7)$ & $1.850(6)$ \\
\hline $\mathrm{Sb}(2)-\mathrm{F}(12)$ & $1.853(6)$ \\
\hline $\mathrm{Sb}(2)-\mathrm{F}(9)$ & $1.859(6)$ \\
\hline $\mathrm{Sb}(2)-\mathrm{F}(11)$ & $1.873(6)$ \\
\hline $\mathrm{Sb}(2)-\mathrm{F}(8)$ & $1.875(6)$ \\
\hline $\mathrm{Sb}(2)-\mathrm{F}(10)$ & $1.884(6)$ \\
\hline$S(4)-C(16)$ & $1.761(9)$ \\
\hline$S(4)-C(13)$ & $1.786(8)$ \\
\hline$S(2)-C(5)$ & $1.743(9)$ \\
\hline$S(2)-C(8)$ & $1.756(9)$ \\
\hline$S(3)-C(9)$ & $1.755(9)$ \\
\hline$S(3)-C(12)$ & $1.771(9)$ \\
\hline$S(6)-C(24)$ & $1.718(9)$ \\
\hline$S(6)-C(21)$ & $1.739(9)$ \\
\hline$S(5)-C(20)$ & $1.755(9)$ \\
\hline$S(5)-C(17)$ & $1.764(9)$ \\
\hline$S(1)-C(1)$ & $1.722(9)$ \\
\hline$S(1)-C(4)$ & $1.777(9)$ \\
\hline $\mathrm{Cl}(2)-\mathrm{C}(61)$ & $1.692(10)$ \\
\hline $\mathrm{Cl}(1)-\mathrm{C}(61)$ & $1.708(10)$ \\
\hline $\mathrm{C}(19)-\mathrm{C}(20)$ & $1.384(12)$ \\
\hline $\mathrm{C}(19)-\mathrm{C}(18)$ & $1.405(12)$ \\
\hline $\mathrm{C}(19)-\mathrm{C}(52)$ & $1.498(12)$ \\
\hline $\mathrm{C}(25)-\mathrm{C}(2)$ & $1.514(13)$ \\
\hline$C(25)-C(26)$ & $1.541(12)$ \\
\hline$C(25)-C(27)$ & $1.556(13)$ \\
\hline$C(7)-C(6)$ & $1.408(11)$ \\
\hline$C(7)-C(8)$ & $1.416(11)$ \\
\hline $\mathrm{C}(7)-\mathrm{C}(34)$ & $1.514(12)$ \\
\hline$C(14)-C(15)$ & $1.359(12)$ \\
\hline $\mathrm{C}(14)-\mathrm{C}(13)$ & $1.420(12)$ \\
\hline
\end{tabular}




\begin{tabular}{|c|c|}
\hline$C(14)-C(43)$ & $1.505(12)$ \\
\hline $\mathrm{C}(46)-\mathrm{C}(15)$ & $1.529(11)$ \\
\hline$C(46)-C(47)$ & $1.538(12)$ \\
\hline$C(46)-C(48)$ & $1.552(12)$ \\
\hline$C(15)-C(16)$ & $1.442(11)$ \\
\hline$C(49)-C(18)$ & $1.505(11)$ \\
\hline$C(49)-C(50)$ & $1.533(12)$ \\
\hline $\mathrm{C}(49)-\mathrm{C}(51)$ & $1.562(11)$ \\
\hline $\mathrm{C}(37)-\mathrm{C}(10)$ & $1.503(12)$ \\
\hline C(37)-C(39) & $1.545(12)$ \\
\hline $\mathrm{C}(37)-\mathrm{C}(38)$ & $1.558(11)$ \\
\hline$C(52)-C(54)$ & $1.524(12)$ \\
\hline$C(52)-C(53)$ & $1.539(12)$ \\
\hline $\mathrm{C}(9)-\mathrm{C}(8)$ & $1.408(12)$ \\
\hline C(9)-C(10) & $1.425(12)$ \\
\hline$C(17)-C(16)$ & $1.415(11)$ \\
\hline $\mathrm{C}(17)-\mathrm{C}(18)$ & $1.423(12)$ \\
\hline$C(5)-C(6)$ & $1.405(12)$ \\
\hline$C(5)-C(4)$ & $1.440(12)$ \\
\hline $\mathrm{C}(54)-\mathrm{C}(51)$ & $1.571(12)$ \\
\hline C(39)-C(41) & $1.544(12)$ \\
\hline $\mathrm{C}(11)-\mathrm{C}(10)$ & $1.400(12)$ \\
\hline $\mathrm{C}(11)-\mathrm{C}(12)$ & $1.436(12)$ \\
\hline$C(11)-C(40)$ & $1.512(12)$ \\
\hline $\mathrm{C}(2)-\mathrm{C}(1)$ & $1.358(12)$ \\
\hline$C(2)-C(3)$ & $1.418(12)$ \\
\hline $\mathrm{C}(43)-\mathrm{C}(45)$ & $1.552(13)$ \\
\hline $\mathrm{C}(43)-\mathrm{C}(44)$ & $1.568(12)$ \\
\hline $\mathrm{C}(13)-\mathrm{C}(12)$ & $1.409(12)$ \\
\hline $\mathrm{C}(24)-\mathrm{C}(23)$ & $1.374(12)$ \\
\hline $\mathrm{C}(24)-\mathrm{C}(58)$ & $1.502(12)$ \\
\hline $\mathrm{C}(20)-\mathrm{C}(21)$ & $1.433(12)$ \\
\hline
\end{tabular}




\begin{tabular}{|c|c|}
\hline$C(34)-C(36)$ & $1.532(13)$ \\
\hline $\mathrm{C}(34)-\mathrm{C}(35)$ & $1.545(12)$ \\
\hline$C(23)-C(22)$ & $1.415(12)$ \\
\hline$C(23)-C(55)$ & $1.501(12)$ \\
\hline$C(47)-C(45)$ & $1.569(12)$ \\
\hline$C(56)-C(55)$ & $1.536(13)$ \\
\hline$C(56)-C(60)$ & $1.586(13)$ \\
\hline $\mathrm{C}(40)-\mathrm{C}(41)$ & $1.539(12)$ \\
\hline$C(40)-C(42)$ & $1.554(12)$ \\
\hline$C(53)-C(50)$ & $1.554(12)$ \\
\hline $\mathrm{C}(31)-\mathrm{C}(33)$ & $1.499(12)$ \\
\hline$C(31)-C(6)$ & $1.521(12)$ \\
\hline $\mathrm{C}(31)-\mathrm{C}(32)$ & $1.551(12)$ \\
\hline $\mathrm{C}(21)-\mathrm{C}(22)$ & $1.407(12)$ \\
\hline $\mathrm{C}(44)-\mathrm{C}(48)$ & $1.569(12)$ \\
\hline$C(26)-C(29)$ & $1.533(12)$ \\
\hline $\mathrm{C}(1)-\mathrm{C}(28)$ & $1.492(13)$ \\
\hline $\mathrm{C}(29)-\mathrm{C}(28)$ & $1.575(12)$ \\
\hline $\mathrm{C}(30)-\mathrm{C}(27)$ & $1.529(12)$ \\
\hline $\mathrm{C}(30)-\mathrm{C}(28)$ & $1.570(12)$ \\
\hline $\mathrm{C}(32)-\mathrm{C}(35)$ & $1.534(12)$ \\
\hline$C(33)-C(36)$ & $1.539(13)$ \\
\hline$C(3)-C(4)$ & $1.391(12)$ \\
\hline$C(57)-C(55)$ & $1.530(12)$ \\
\hline C(57)-C(59) & $1.558(12)$ \\
\hline $\mathrm{C}(59)-\mathrm{C}(58)$ & $1.585(13)$ \\
\hline $\mathrm{C}(38)-\mathrm{C}(42)$ & $1.561(13)$ \\
\hline$C(58)-C(60)$ & $1.526(13)$ \\
\hline $\mathrm{F}(6)-\mathrm{Sb}(1)-\mathrm{F}(1)$ & $89.6(3)$ \\
\hline $\mathrm{F}(6)-\mathrm{Sb}(1)-\mathrm{F}(2)$ & $178.8(3)$ \\
\hline $\mathrm{F}(1)-\mathrm{Sb}(1)-\mathrm{F}(2)$ & $89.5(3)$ \\
\hline
\end{tabular}




\begin{tabular}{|c|c|}
\hline $\mathrm{F}(6)-\mathrm{Sb}(1)-\mathrm{F}(4)$ & $90.4(3)$ \\
\hline $\mathrm{F}(1)-\mathrm{Sb}(1)-\mathrm{F}(4)$ & $179.8(3)$ \\
\hline $\mathrm{F}(2)-\mathrm{Sb}(1)-\mathrm{F}(4)$ & $90.6(3)$ \\
\hline $\mathrm{F}(6)-\mathrm{Sb}(1)-\mathrm{F}(5)$ & $91.7(3)$ \\
\hline $\mathrm{F}(1)-\mathrm{Sb}(1)-\mathrm{F}(5)$ & $90.5(3)$ \\
\hline $\mathrm{F}(2)-\mathrm{Sb}(1)-\mathrm{F}(5)$ & $89.1(3)$ \\
\hline $\mathrm{F}(4)-\mathrm{Sb}(1)-\mathrm{F}(5)$ & $89.7(3)$ \\
\hline $\mathrm{F}(6)-\mathrm{Sb}(1)-\mathrm{F}(3)$ & $88.6(3)$ \\
\hline $\mathrm{F}(1)-\mathrm{Sb}(1)-\mathrm{F}(3)$ & $91.3(3)$ \\
\hline $\mathrm{F}(2)-\mathrm{Sb}(1)-\mathrm{F}(3)$ & $90.7(2)$ \\
\hline $\mathrm{F}(4)-\mathrm{Sb}(1)-\mathrm{F}(3)$ & $88.5(3)$ \\
\hline $\mathrm{F}(5)-\mathrm{Sb}(1)-\mathrm{F}(3)$ & $178.2(3)$ \\
\hline $\mathrm{F}(7)-\mathrm{Sb}(2)-\mathrm{F}(12)$ & $90.4(3)$ \\
\hline $\mathrm{F}(7)-\mathrm{Sb}(2)-\mathrm{F}(9)$ & $90.5(3)$ \\
\hline $\mathrm{F}(12)-\mathrm{Sb}(2)-\mathrm{F}(9)$ & $179.0(3)$ \\
\hline $\mathrm{F}(7)-\mathrm{Sb}(2)-\mathrm{F}(11)$ & $90.9(3)$ \\
\hline $\mathrm{F}(12)-\mathrm{Sb}(2)-\mathrm{F}(11)$ & $90.4(3)$ \\
\hline $\mathrm{F}(9)-\mathrm{Sb}(2)-\mathrm{F}(11)$ & $90.0(3)$ \\
\hline $\mathrm{F}(7)-\mathrm{Sb}(2)-\mathrm{F}(8)$ & $88.4(3)$ \\
\hline $\mathrm{F}(12)-\mathrm{Sb}(2)-\mathrm{F}(8)$ & $89.7(3)$ \\
\hline $\mathrm{F}(9)-\mathrm{Sb}(2)-\mathrm{F}(8)$ & $89.8(3)$ \\
\hline $\mathrm{F}(11)-\mathrm{Sb}(2)-\mathrm{F}(8)$ & 179.3(3) \\
\hline $\mathrm{F}(7)-\mathrm{Sb}(2)-\mathrm{F}(10)$ & $177.8(3)$ \\
\hline $\mathrm{F}(12)-\mathrm{Sb}(2)-\mathrm{F}(10)$ & $90.1(3)$ \\
\hline $\mathrm{F}(9)-\mathrm{Sb}(2)-\mathrm{F}(10)$ & $89.0(3)$ \\
\hline $\mathrm{F}(11)-\mathrm{Sb}(2)-\mathrm{F}(10)$ & $91.1(3)$ \\
\hline $\mathrm{F}(8)-\mathrm{Sb}(2)-\mathrm{F}(10)$ & $89.5(3)$ \\
\hline $\mathrm{C}(16)-\mathrm{S}(4)-\mathrm{C}(13)$ & $92.4(4)$ \\
\hline $\mathrm{C}(5)-\mathrm{S}(2)-\mathrm{C}(8)$ & $93.0(4)$ \\
\hline $\mathrm{C}(9)-\mathrm{S}(3)-\mathrm{C}(12)$ & $93.1(4)$ \\
\hline $\mathrm{C}(24)-\mathrm{S}(6)-\mathrm{C}(21)$ & $91.0(5)$ \\
\hline $\mathrm{C}(20)-\mathrm{S}(5)-\mathrm{C}(17)$ & $92.4(4)$ \\
\hline
\end{tabular}




\begin{tabular}{|c|c|}
\hline $\mathrm{C}(1)-\mathrm{S}(1)-\mathrm{C}(4)$ & $90.5(4)$ \\
\hline$C(20)-C(19)-C(18)$ & $113.6(8)$ \\
\hline $\mathrm{C}(20)-\mathrm{C}(19)-\mathrm{C}(52)$ & 133.1(9) \\
\hline$C(18)-C(19)-C(52)$ & $112.9(8)$ \\
\hline$C(2)-C(25)-C(26)$ & $108.6(8)$ \\
\hline $\mathrm{C}(2)-\mathrm{C}(25)-\mathrm{C}(27)$ & $106.9(7)$ \\
\hline$C(26)-C(25)-C(27)$ & $105.7(8)$ \\
\hline $\mathrm{C}(6)-\mathrm{C}(7)-\mathrm{C}(8)$ & $114.0(8)$ \\
\hline$C(6)-C(7)-C(34)$ & $112.1(7)$ \\
\hline $\mathrm{C}(8)-\mathrm{C}(7)-\mathrm{C}(34)$ & $133.4(8)$ \\
\hline$C(15)-C(14)-C(13)$ & $115.3(8)$ \\
\hline $\mathrm{C}(15)-\mathrm{C}(14)-\mathrm{C}(43)$ & $114.9(8)$ \\
\hline$C(13)-C(14)-C(43)$ & $129.6(8)$ \\
\hline$C(15)-C(46)-C(47)$ & $107.0(7)$ \\
\hline$C(15)-C(46)-C(48)$ & $105.5(7)$ \\
\hline $\mathrm{C}(47)-\mathrm{C}(46)-\mathrm{C}(48)$ & $109.9(7)$ \\
\hline$C(14)-C(15)-C(16)$ & $114.3(8)$ \\
\hline$C(14)-C(15)-C(46)$ & $114.3(7)$ \\
\hline$C(16)-C(15)-C(46)$ & $130.9(8)$ \\
\hline$C(18)-C(49)-C(50)$ & $108.8(7)$ \\
\hline $\mathrm{C}(18)-\mathrm{C}(49)-\mathrm{C}(51)$ & $106.9(7)$ \\
\hline$C(50)-C(49)-C(51)$ & $107.1(7)$ \\
\hline $\mathrm{C}(10)-\mathrm{C}(37)-\mathrm{C}(39)$ & $106.0(7)$ \\
\hline $\mathrm{C}(10)-\mathrm{C}(37)-\mathrm{C}(38)$ & $108.5(8)$ \\
\hline $\mathrm{C}(39)-\mathrm{C}(37)-\mathrm{C}(38)$ & $108.1(7)$ \\
\hline$C(19)-C(52)-C(54)$ & $109.2(7)$ \\
\hline$C(19)-C(52)-C(53)$ & $108.5(7)$ \\
\hline $\mathrm{C}(54)-\mathrm{C}(52)-\mathrm{C}(53)$ & $108.3(8)$ \\
\hline$C(8)-C(9)-C(10)$ & $128.9(8)$ \\
\hline $\mathrm{C}(8)-\mathrm{C}(9)-\mathrm{S}(3)$ & $121.3(7)$ \\
\hline $\mathrm{C}(10)-\mathrm{C}(9)-\mathrm{S}(3)$ & $109.7(7)$ \\
\hline$C(16)-C(17)-C(18)$ & $131.6(8)$ \\
\hline
\end{tabular}




\begin{tabular}{|c|c|}
\hline$C(16)-C(17)-S(5)$ & $119.5(7)$ \\
\hline $\mathrm{C}(18)-\mathrm{C}(17)-\mathrm{S}(5)$ & $108.9(7)$ \\
\hline$C(6)-C(5)-C(4)$ & 132.2(9) \\
\hline$C(6)-C(5)-S(2)$ & $110.5(7)$ \\
\hline$C(4)-C(5)-S(2)$ & $117.1(7)$ \\
\hline$C(52)-C(54)-C(51)$ & $108.6(7)$ \\
\hline $\mathrm{C}(41)-\mathrm{C}(39)-\mathrm{C}(37)$ & $110.4(8)$ \\
\hline$C(10)-C(11)-C(12)$ & $113.5(8)$ \\
\hline$C(10)-C(11)-C(40)$ & $113.5(7)$ \\
\hline $\mathrm{C}(12)-\mathrm{C}(11)-\mathrm{C}(40)$ & $132.5(8)$ \\
\hline $\mathrm{C}(1)-\mathrm{C}(2)-\mathrm{C}(3)$ & $114.0(9)$ \\
\hline$C(1)-C(2)-C(25)$ & $113.2(8)$ \\
\hline$C(3)-C(2)-C(25)$ & $132.5(8)$ \\
\hline$C(14)-C(43)-C(45)$ & $107.5(8)$ \\
\hline$C(14)-C(43)-C(44)$ & $106.3(7)$ \\
\hline$C(45)-C(43)-C(44)$ & $108.5(8)$ \\
\hline$C(17)-C(16)-C(15)$ & $130.3(8)$ \\
\hline$C(17)-C(16)-S(4)$ & $120.5(6)$ \\
\hline$C(15)-C(16)-S(4)$ & $109.2(6)$ \\
\hline$C(12)-C(13)-C(14)$ & $131.8(8)$ \\
\hline $\mathrm{C}(12)-\mathrm{C}(13)-\mathrm{S}(4)$ & $119.4(7)$ \\
\hline $\mathrm{C}(14)-\mathrm{C}(13)-\mathrm{S}(4)$ & $108.7(6)$ \\
\hline $\mathrm{C}(23)-\mathrm{C}(24)-\mathrm{C}(58)$ & $115.3(8)$ \\
\hline$C(23)-C(24)-S(6)$ & $113.9(7)$ \\
\hline$C(58)-C(24)-S(6)$ & $130.7(7)$ \\
\hline$C(19)-C(20)-C(21)$ & 131.3(9) \\
\hline$C(19)-C(20)-S(5)$ & $110.8(7)$ \\
\hline$C(21)-C(20)-S(5)$ & $117.9(7)$ \\
\hline$C(7)-C(34)-C(36)$ & $107.6(8)$ \\
\hline$C(7)-C(34)-C(35)$ & $108.2(7)$ \\
\hline$C(36)-C(34)-C(35)$ & $106.5(7)$ \\
\hline $\mathrm{C}(24)-\mathrm{C}(23)-\mathrm{C}(22)$ & 111.1(8) \\
\hline
\end{tabular}




\begin{tabular}{|c|c|}
\hline$C(24)-C(23)-C(55)$ & $114.2(8)$ \\
\hline$C(22)-C(23)-C(55)$ & $134.1(8)$ \\
\hline $\mathrm{C}(11)-\mathrm{C}(10)-\mathrm{C}(9)$ & $114.4(8)$ \\
\hline $\mathrm{C}(11)-\mathrm{C}(10)-\mathrm{C}(37)$ & $113.8(8)$ \\
\hline $\mathrm{C}(9)-\mathrm{C}(10)-\mathrm{C}(37)$ & $131.3(8)$ \\
\hline $\mathrm{C}(9)-\mathrm{C}(8)-\mathrm{C}(7)$ & $130.6(8)$ \\
\hline $\mathrm{C}(9)-\mathrm{C}(8)-\mathrm{S}(2)$ & $120.1(7)$ \\
\hline$C(7)-C(8)-S(2)$ & $109.2(6)$ \\
\hline$C(46)-C(47)-C(45)$ & $110.1(8)$ \\
\hline$C(55)-C(56)-C(60)$ & $111.0(8)$ \\
\hline$C(11)-C(40)-C(41)$ & $107.2(7)$ \\
\hline$C(11)-C(40)-C(42)$ & $107.7(7)$ \\
\hline$C(41)-C(40)-C(42)$ & $108.7(8)$ \\
\hline$C(52)-C(53)-C(50)$ & $109.4(7)$ \\
\hline $\mathrm{C}(40)-\mathrm{C}(41)-\mathrm{C}(39)$ & $109.9(7)$ \\
\hline$C(33)-C(31)-C(6)$ & $106.6(7)$ \\
\hline $\mathrm{C}(33)-\mathrm{C}(31)-\mathrm{C}(32)$ & $108.4(8)$ \\
\hline$C(6)-C(31)-C(32)$ & $107.6(8)$ \\
\hline $\mathrm{C}(49)-\mathrm{C}(50)-\mathrm{C}(53)$ & $110.0(7)$ \\
\hline$C(22)-C(21)-C(20)$ & $125.7(8)$ \\
\hline $\mathrm{C}(22)-\mathrm{C}(21)-\mathrm{S}(6)$ & $110.6(7)$ \\
\hline$C(20)-C(21)-S(6)$ & $123.7(7)$ \\
\hline $\mathrm{C}(19)-\mathrm{C}(18)-\mathrm{C}(17)$ & $113.9(8)$ \\
\hline $\mathrm{C}(19)-\mathrm{C}(18)-\mathrm{C}(49)$ & $113.6(8)$ \\
\hline $\mathrm{C}(17)-\mathrm{C}(18)-\mathrm{C}(49)$ & $132.5(8)$ \\
\hline $\mathrm{C}(43)-\mathrm{C}(45)-\mathrm{C}(47)$ & $109.8(7)$ \\
\hline $\mathrm{C}(48)-\mathrm{C}(44)-\mathrm{C}(43)$ & $109.2(7)$ \\
\hline$C(29)-C(26)-C(25)$ & 111.2(7) \\
\hline $\mathrm{C}(2)-\mathrm{C}(1)-\mathrm{C}(28)$ & 117.1(9) \\
\hline $\mathrm{C}(2)-\mathrm{C}(1)-\mathrm{S}(1)$ & $112.8(7)$ \\
\hline $\mathrm{C}(28)-\mathrm{C}(1)-\mathrm{S}(1)$ & $129.9(7)$ \\
\hline$C(5)-C(6)-C(7)$ & $113.2(8)$ \\
\hline
\end{tabular}




\begin{tabular}{|c|c|}
\hline$C(5)-C(6)-C(31)$ & $132.1(8)$ \\
\hline$C(7)-C(6)-C(31)$ & $114.4(8)$ \\
\hline $\mathrm{C}(13)-\mathrm{C}(12)-\mathrm{C}(11)$ & $130.8(8)$ \\
\hline $\mathrm{C}(13)-\mathrm{C}(12)-\mathrm{S}(3)$ & $119.9(7)$ \\
\hline $\mathrm{C}(11)-\mathrm{C}(12)-\mathrm{S}(3)$ & 109.2(7) \\
\hline $\mathrm{C}(21)-\mathrm{C}(22)-\mathrm{C}(23)$ & $113.2(8)$ \\
\hline $\mathrm{Cl}(2)-\mathrm{C}(61)-\mathrm{Cl}(1)$ & $115.8(7)$ \\
\hline $\mathrm{C}(26)-\mathrm{C}(29)-\mathrm{C}(28)$ & $110.1(7)$ \\
\hline $\mathrm{C}(27)-\mathrm{C}(30)-\mathrm{C}(28)$ & $111.1(8)$ \\
\hline $\mathrm{C}(1)-\mathrm{C}(28)-\mathrm{C}(30)$ & $105.1(7)$ \\
\hline $\mathrm{C}(1)-\mathrm{C}(28)-\mathrm{C}(29)$ & $106.0(8)$ \\
\hline $\mathrm{C}(30)-\mathrm{C}(28)-\mathrm{C}(29)$ & $107.0(8)$ \\
\hline$C(35)-C(32)-C(31)$ & $110.2(7)$ \\
\hline $\mathrm{C}(31)-\mathrm{C}(33)-\mathrm{C}(36)$ & $110.1(8)$ \\
\hline $\mathrm{C}(4)-\mathrm{C}(3)-\mathrm{C}(2)$ & $111.7(8)$ \\
\hline $\mathrm{C}(49)-\mathrm{C}(51)-\mathrm{C}(54)$ & $109.9(7)$ \\
\hline $\mathrm{C}(55)-\mathrm{C}(57)-\mathrm{C}(59)$ & $110.6(8)$ \\
\hline $\mathrm{C}(57)-\mathrm{C}(59)-\mathrm{C}(58)$ & $110.0(7)$ \\
\hline$C(3)-C(4)-C(5)$ & $126.1(8)$ \\
\hline $\mathrm{C}(3)-\mathrm{C}(4)-\mathrm{S}(1)$ & $110.9(7)$ \\
\hline$C(5)-C(4)-S(1)$ & $121.9(7)$ \\
\hline $\mathrm{C}(37)-\mathrm{C}(38)-\mathrm{C}(42)$ & $110.0(7)$ \\
\hline $\mathrm{C}(32)-\mathrm{C}(35)-\mathrm{C}(34)$ & $110.4(8)$ \\
\hline$C(23)-C(55)-C(57)$ & $107.6(8)$ \\
\hline$C(23)-C(55)-C(56)$ & $106.4(7)$ \\
\hline$C(57)-C(55)-C(56)$ & $107.4(8)$ \\
\hline$C(30)-C(27)-C(25)$ & $110.3(8)$ \\
\hline$C(24)-C(58)-C(60)$ & $108.2(8)$ \\
\hline $\mathrm{C}(24)-\mathrm{C}(58)-\mathrm{C}(59)$ & $104.8(8)$ \\
\hline$C(60)-C(58)-C(59)$ & $107.5(8)$ \\
\hline $\mathrm{C}(34)-\mathrm{C}(36)-\mathrm{C}(33)$ & $111.1(8)$ \\
\hline $\mathrm{C}(40)-\mathrm{C}(42)-\mathrm{C}(38)$ & $109.3(7)$ \\
\hline
\end{tabular}


Symmetry transformations used to generate equivalent atoms: 
Table S13. Cartesian Coordinates from the Optimized Structure of $1(2 \mathrm{~T})^{+}$at B3LYP/6-31G(d)

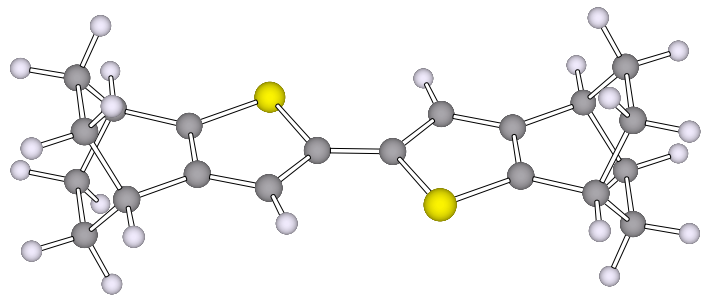

\begin{tabular}{|c|c|c|c|c|c|}
\hline \multirow{2}{*}{$\begin{array}{l}\text { Center } \\
\text { Number }\end{array}$} & Atomic & \multicolumn{2}{|c|}{ Atomic } & \multicolumn{2}{|c|}{ Coordinates (Angstroms } \\
\hline & . Number & & Type & $X$ & Z \\
\hline 1 & 6 & 0 & -5.180863 & 0.615153 & -1.261784 \\
\hline 2 & 6 & 0 & -4.490980 & 1.225573 & -0.000246 \\
\hline 3 & 6 & 0 & -5.041435 & -0.936343 & 1.258672 \\
\hline 4 & 6 & 0 & -2.938324 & -0.674592 & 0.000318 \\
\hline 5 & 6 & 0 & -4.254299 & -1.402893 & 0.000342 \\
\hline 6 & 1 & 0 & -4.742154 & 1.047727 & 2.166344 \\
\hline 7 & 6 & 0 & -5.180589 & 0.615729 & 1.261765 \\
\hline 8 & 1 & 0 & -4.531542 & -1.279583 & 2.164175 \\
\hline 9 & 6 & 0 & -1.606545 & -1.071384 & 0.000388 \\
\hline 10 & 1 & & & -2.488620 & 0.000587 \\
\hline 11 & 6 & & -3.088792 & 0.723744 & -0.000127 \\
\hline 12 & 1 & & -6.233019 & 0.917416 & 1.241205 \\
\hline 13 & 1 & & & & -0.000457 \\
\hline 14 & 1 & 0 & -6.026202 & -1.414642 & 1.247728 \\
\hline 15 & 6 & & -0.702384 & 0.015821 & 0.000123 \\
\hline 16 & 1 & 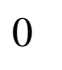 & -4.529861 & -1.279780 & -2.163632 \\
\hline 17 & 16 & 0 & -1.586511 & 1.559542 & -0.000369 \\
\hline 18 & 6 & 0 & -5.040793 & -0.936848 & -1.258588 \\
\hline 19 & 1 & & -4.743313 & 1.047276 & -2.166725 \\
\hline 20 & 1 & 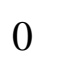 & -6.233465 & 0.916218 & -1.240576 \\
\hline 21 & 1 & 0 & -6.025278 & -1.415727 & -1.248523 \\
\hline 22 & 6 & & 0.702384 & -0.015780 & 0.000121 \\
\hline 23 & 1 & & 1.273824 & 2.104389 & 0.000645 \\
\hline 24 & 6 & 0 & 1.606559 & 1.071413 & 0.000379 \\
\hline 25 & 6 & 0 & 3.088783 & -0.723736 & -0.000127 \\
\hline 26 & 1 & 0 & 6.233457 & -0.916266 & -1.240564 \\
\hline 27 & 1 & 0 & -1.273798 & -2.104356 & 0.000656 \\
\hline 28 & 6 & 0 & 2.938335 & 0.674602 & 0.000311 \\
\hline 29 & 16 & & 1.586489 & -1.559512 & -0.00037 \\
\hline
\end{tabular}




$\begin{array}{llllll}30 & 6 & 0 & 4.254323 & 1.402881 & 0.000337 \\ 31 & 1 & 0 & 4.560782 & -2.316081 & -0.000448 \\ 32 & 6 & 0 & 5.040815 & 0.936820 & -1.258588 \\ 33 & 1 & 0 & 4.123946 & 2.488611 & 0.000578 \\ 34 & 6 & 0 & 5.041449 & 0.936323 & 1.258671 \\ 35 & 1 & 0 & 4.531561 & 1.279576 & 2.164172 \\ 36 & 1 & 0 & 6.026225 & 1.414604 & 1.247726 \\ 37 & 6 & 0 & 5.180577 & -0.615751 & 1.261770 \\ 38 & 6 & 0 & 4.490963 & -1.225588 & -0.000241 \\ 39 & 1 & 0 & 4.742133 & -1.047739 & 2.166349 \\ 40 & 1 & 0 & 6.233002 & -0.917454 & 1.241214 \\ 41 & 1 & 0 & 4.529896 & 1.279758 & -2.163637 \\ 42 & 6 & 0 & 5.180860 & -0.615184 & -1.261778 \\ 43 & 1 & 0 & 6.025310 & 1.415680 & -1.248518 \\ 44 & 1 & 0 & 4.743307 & -1.047304 & -2.166720\end{array}$

$\mathrm{HF}=-1571.5250434$ hartree

Table S14. Cartesian Coordinates from the Optimized Structure of $\mathbf{1}(2 \mathrm{~T})^{2+}$ (transoid) at B3LYP/6$31 \mathrm{G}(\mathrm{d})$

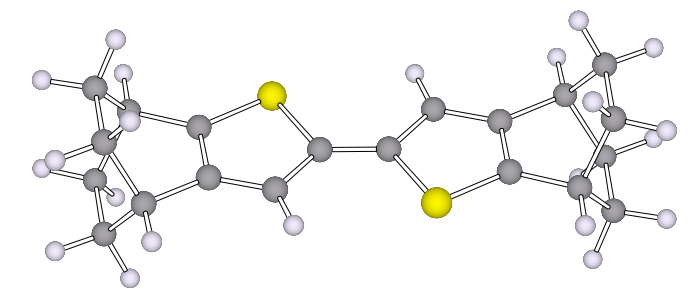

\begin{tabular}{ccccccc} 
Center & \multicolumn{2}{c}{ Atomic } & \multicolumn{2}{c}{ Atomic } & \multicolumn{3}{c}{ Coordinates (Angstroms) } \\
Number & \multicolumn{2}{c}{ Number } & Type & X & Y & Z \\
\hline------------------------------- \\
1 & 6 & 0 & 0.629070 & 5.132124 & 1.270623 \\
2 & 6 & 0 & -0.148005 & 4.606254 & 0.001060 \\
3 & 6 & 0 & 2.105028 & 4.642602 & -1.263610 \\
4 & 6 & 0 & 1.378752 & 2.667867 & -0.000513 \\
5 & 6 & 0 & 2.385502 & 3.777811 & 0.000149 \\
6 & 1 & 0 & 0.102573 & 4.823110 & -2.174337 \\
7 & 6 & 0 & 0.628752 & 5.133921 & -1.267960
\end{tabular}




$\begin{array}{cccccc}8 & 1 & 0 & 2.321955 & 4.067528 & -2.168593 \\ 9 & 6 & 0 & 1.445875 & 1.303632 & -0.001404 \\ 10 & 1 & 0 & 3.409703 & 3.397667 & -0.000253 \\ 11 & 6 & 0 & 0.011435 & 3.150503 & -0.000031 \\ 12 & 1 & 0 & 0.566109 & 6.225216 & -1.217696 \\ 13 & 1 & 0 & -1.187378 & 4.939350 & 0.001427 \\ 14 & 1 & 0 & 2.796588 & 5.489805 & -1.254138 \\ 15 & 6 & 0 & 0.148005 & 0.667937 & -0.001280 \\ 16 & 1 & 0 & 2.322521 & 4.064483 & 2.169308 \\ 17 & 16 & 0 & -1.158624 & 1.915498 & -0.001418 \\ 18 & 6 & 0 & 2.105351 & 4.640830 & 1.265192 \\ 19 & 1 & 0 & 0.103131 & 4.820008 & 2.176689 \\ 20 & 1 & 0 & 0.566402 & 6.223489 & 1.221937 \\ 21 & 1 & 0 & 2.796895 & 5.488056 & 1.256742 \\ 22 & 6 & 0 & -0.148005 & -0.667937 & -0.001280 \\ 23 & 1 & 0 & -2.369534 & -0.733043 & -0.001747 \\ 24 & 6 & 0 & -1.445875 & -1.303632 & -0.001404 \\ 25 & 6 & 0 & -0.011435 & -3.150503 & -0.000031 \\ 26 & 1 & 0 & -0.566402 & -6.223489 & 1.221937 \\ 27 & 1 & 0 & 2.369534 & 0.733043 & -0.001747 \\ 28 & 6 & 0 & -1.378752 & -2.667867 & -0.000513 \\ 29 & 16 & 0 & 1.158624 & -1.915498 & -0.001418 \\ 30 & 6 & 0 & -2.385502 & -3.777811 & 0.000149 \\ 31 & 1 & 0 & 1.187378 & -4.939350 & 0.001427 \\ 32 & 6 & 0 & -2.105351 & -4.640830 & 1.265192 \\ 33 & 1 & 0 & -3.409703 & -3.397667 & -0.000253 \\ 34 & 6 & 0 & -2.105028 & -4.642602 & -1.263610 \\ 35 & 1 & 0 & -2.321955 & -4.067528 & -2.168593 \\ 36 & 1 & 0 & -2.796588 & -5.489805 & -1.254138 \\ 37 & 6 & 0 & -0.628752 & -5.133921 & -1.267960 \\ 38 & 6 & 0 & 0.148005 & -4.606254 & 0.001060 \\ 39 & 1 & 0 & -0.102573 & -4.823110 & -2.174337 \\ 40 & 1 & 0 & -0.566109 & -6.225216 & -1.217696 \\ 41 & 1 & 0 & -2.322521 & -4.064483 & 2.169308 \\ 42 & 6 & 0 & -0.629070 & -5.132124 & 1.270623 \\ 43 & 1 & 0 & -2.796895 & -5.488056 & 1.256742 \\ 44 & 1 & 0 & -0.103131 & -4.820008 & 2.176689 \\ -------------------------------------------------------\end{array}$

$\mathrm{HF}=-1571.1466953$ hartree 
Table S15. Cartesian Coordinates from the Optimized Structure of $\mathbf{1}(2 \mathrm{~T})^{2+}$ (cisoid) at B3LYP/6-31G(d)

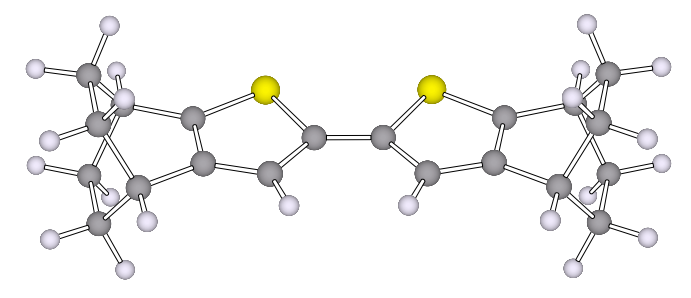

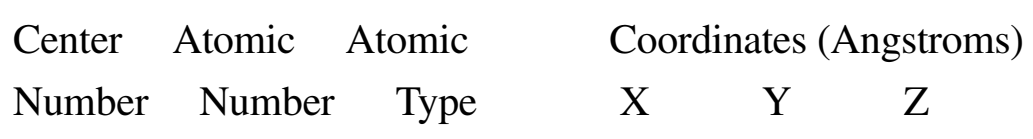

$\begin{array}{cccccc}-------------------------------------------------- \\ 1 & 6 & 0 & -1.270307 & 5.156506 & -0.421742 \\ 2 & 6 & 0 & -0.000005 & 4.488965 & -1.079685 \\ 3 & 6 & 0 & 1.263366 & 4.968835 & 1.122623 \\ 4 & 6 & 0 & 0.000028 & 2.888324 & 0.798271 \\ 5 & 6 & 0 & 0.000009 & 4.175507 & 1.567145 \\ 6 & 1 & 0 & 2.175251 & 4.742610 & -0.874219 \\ 7 & 6 & 0 & 1.270261 & 5.156577 & -0.421744 \\ 8 & 1 & 0 & 2.169080 & 4.450844 & 1.451536 \\ 9 & 6 & 0 & 0.000048 & 1.563991 & 1.132751 \\ 10 & 1 & 0 & 0.000016 & 4.004203 & 2.646184 \\ 11 & 6 & 0 & 0.000020 & 3.093743 & -0.636546 \\ 12 & 1 & 0 & 1.225615 & 6.213627 & -0.701345 \\ 13 & 1 & 0 & -0.000007 & 4.611288 & -2.164299 \\ 14 & 1 & 0 & 1.250379 & 5.936841 & 1.631519 \\ 15 & 6 & 0 & 0.000020 & 0.684807 & -0.016603 \\ 16 & 1 & 0 & -2.169069 & 4.450826 & 1.451585 \\ 17 & 16 & 0 & 0.000074 & 1.652260 & -1.540553 \\ 18 & 6 & 0 & -1.263366 & 4.968811 & 1.122632 \\ 19 & 1 & 0 & -2.175276 & 4.742458 & -0.874186 \\ 20 & 1 & 0 & -1.225744 & 6.213551 & -0.701378 \\ 21 & 1 & 0 & -1.250370 & 5.936833 & 1.631498 \\ 22 & 6 & 0 & -0.000020 & -0.684807 & -0.016603 \\ 23 & 1 & 0 & -0.000043 & -1.186606 & 2.149344 \\ 24 & 6 & 0 & -0.000048 & -1.563991 & 1.132751 \\ 25 & 6 & 0 & -0.000020 & -3.093743 & -0.636546 \\ 26 & 1 & 0 & 1.225744 & -6.213551 & -0.701378 \\ 27 & 1 & 0 & 0.000043 & 1.186606 & 2.149344 \\ 28 & 6 & 0 & -0.000028 & -2.888324 & 0.798271 \\ 29 & 16 & 0 & -0.000074 & -1.652260 & -1.540553\end{array}$




\begin{tabular}{|c|c|c|c|c|c|}
\hline 30 & 6 & 0 & -0.000009 & -4.175507 & 1.567145 \\
\hline 31 & 1 & 0 & 0.000007 & -4.611288 & -2.164299 \\
\hline 32 & 6 & 0 & 1.263366 & -4.968811 & 1.122632 \\
\hline 33 & 1 & 0 & -0.000016 & -4.004203 & 2.646184 \\
\hline 34 & 6 & 0 & -1.263366 & -4.968835 & 1.122623 \\
\hline 35 & 1 & 0 & -2.169080 & -4.450844 & 1.451536 \\
\hline 36 & 1 & 0 & -1.250379 & -5.936841 & 1.631519 \\
\hline 37 & 6 & 0 & -1.270261 & -5.156577 & -0.421744 \\
\hline 38 & 6 & 0 & 0.000005 & -4.488965 & -1.079685 \\
\hline 39 & 1 & 0 & -2.175251 & -4.742610 & -0.874219 \\
\hline 40 & 1 & 0 & -1.225615 & -6.213627 & -0.701345 \\
\hline 41 & 1 & 0 & 2.169069 & -4.450826 & 1.451585 \\
\hline 42 & 6 & 0 & 1.270307 & -5.156506 & -0.421742 \\
\hline 43 & 1 & 0 & 1.250370 & -5.936833 & 1.631498 \\
\hline 44 & 1 & 0 & 2.175276 & -4.742458 & -0.874186 \\
\hline
\end{tabular}

$\mathrm{HF}=-1571.1452609$ hartree

Table S16. Cartesian Coordinates from the Optimized Structure of $1(3 \mathrm{~T})^{+}$at B3LYP/6-31G(d)

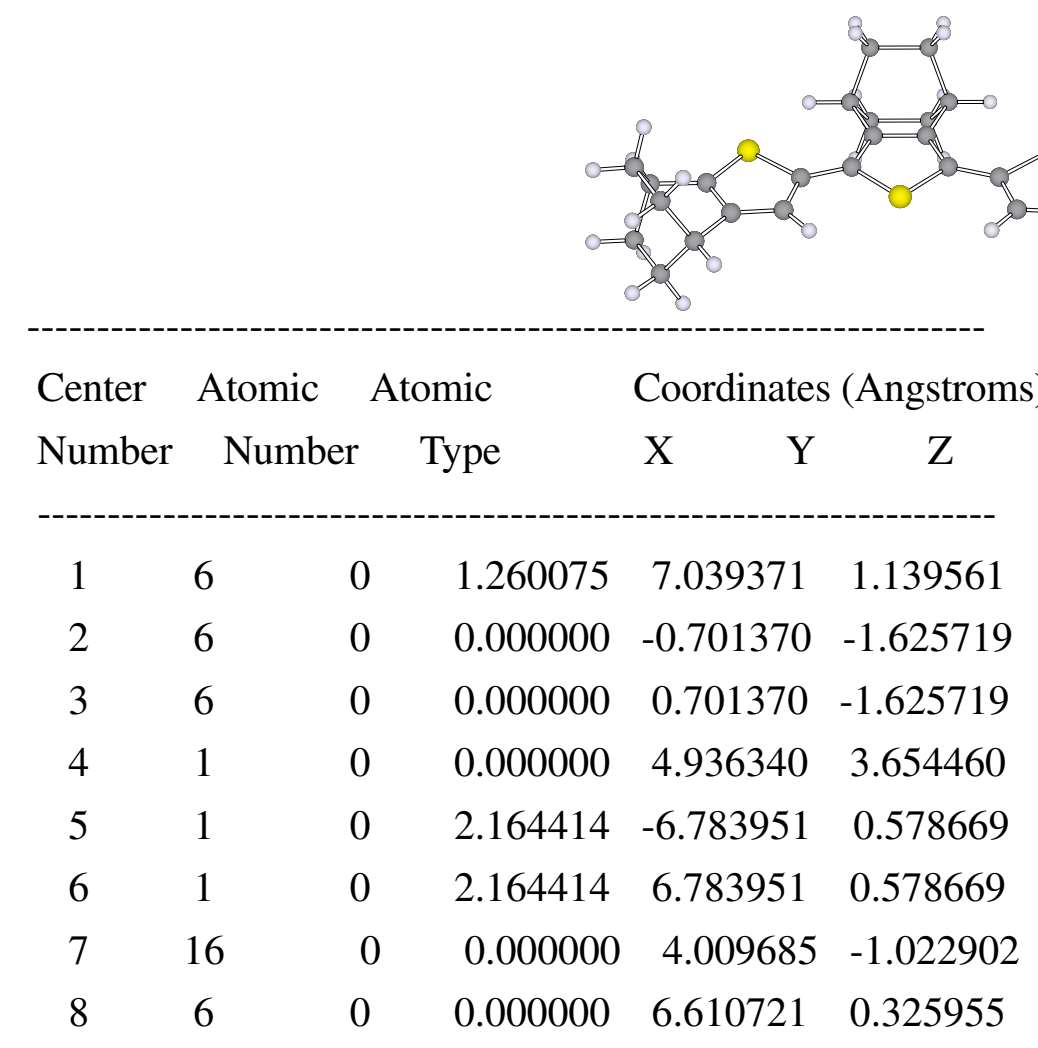




$\begin{array}{cccccc}9 & 1 & 0 & 1.242845 & 8.130080 & 1.238821 \\ 10 & 6 & 0 & -1.260075 & 7.039371 & 1.139561 \\ 11 & 1 & 0 & 0.000000 & 7.065865 & -0.667967 \\ 12 & 1 & 0 & -1.242845 & 8.130080 & 1.238821 \\ 13 & 6 & 0 & -1.257833 & 6.350652 & 2.537677 \\ 14 & 1 & 0 & -2.164414 & 6.783951 & 0.578669 \\ 15 & 6 & 0 & 0.000000 & 4.480803 & 1.532177 \\ 16 & 1 & 0 & -2.162979 & 5.750228 & 2.673012 \\ 17 & 1 & 0 & -1.248725 & 7.098009 & 3.338245 \\ 18 & 1 & 0 & 2.162979 & 5.750228 & 2.673012 \\ 19 & 6 & 0 & 0.000000 & -4.480803 & 1.532177 \\ 20 & 6 & 0 & 1.257833 & 6.350652 & 2.537677 \\ 21 & 6 & 0 & 0.000000 & -2.627439 & 0.092481 \\ 22 & 6 & 0 & 0.000000 & 2.627439 & 0.092481 \\ 23 & 1 & 0 & 1.248725 & -7.098009 & 3.338245 \\ 24 & 1 & 0 & 0.000000 & 2.413665 & 2.273056 \\ 25 & 6 & 0 & -1.261061 & 0.777120 & -3.748645 \\ 26 & 6 & 0 & 0.000000 & -1.277211 & -0.336973 \\ 27 & 1 & 0 & -1.246320 & 1.168900 & -4.771359 \\ 28 & 6 & 0 & 0.000000 & -3.088176 & 1.422858 \\ 29 & 6 & 0 & 0.000000 & 1.305365 & -3.006620 \\ 30 & 16 & 0 & 0.000000 & 0.000000 & 0.885529 \\ 31 & 1 & 0 & 0.000000 & -2.395598 & -3.003077 \\ 32 & 6 & 0 & 0.000000 & 5.117554 & 0.293187 \\ 33 & 1 & 0 & 0.000000 & 2.395598 & -3.003077 \\ 34 & 6 & 0 & 1.261061 & 0.777120 & -3.748645 \\ 35 & 1 & 0 & -2.163591 & 1.169101 & -3.269360 \\ 36 & 1 & 0 & 1.246320 & 1.168900 & -4.771359 \\ 37 & 6 & 0 & 1.261061 & -0.777120 & -3.748645 \\ 38 & 1 & 0 & 2.163591 & 1.169101 & -3.269360 \\ 39 & 6 & 0 & 0.000000 & -1.305365 & -3.006620 \\ 40 & 1 & 0 & 2.163591 & -1.169101 & -3.269360 \\ 41 & 1 & 0 & 1.246320 & -1.168900 & -4.771359 \\ 42 & 6 & 0 & -1.261061 & -0.777120 & -3.748645 \\ 43 & 1 & 0 & -2.163591 & -1.169101 & -3.269360 \\ 44 & 1 & 0 & -1.246320 & -1.168900 & -4.771359 \\ 45 & 6 & 0 & 0.000000 & -6.610721 & 0.325955 \\ 46 & 1 & 0 & 1.248725 & 7.098009 & 3.338245 \\ 47 & 16 & 0 & 0.000000 & -4.009685 & -1.022902\end{array}$




$\begin{array}{rrrrrr}48 & 6 & 0 & 0.000000 & -5.117554 & 0.293187 \\ 49 & 1 & 0 & 2.162979 & -5.750228 & 2.673012 \\ 50 & 6 & 0 & 0.000000 & 5.447323 & 2.687080 \\ 51 & 1 & 0 & 0.000000 & -2.413665 & 2.273056 \\ 52 & 1 & 0 & 0.000000 & -7.065865 & -0.667967 \\ 53 & 6 & 0 & -1.260075 & -7.039371 & 1.139561 \\ 54 & 6 & 0 & 1.260075 & -7.039371 & 1.139561 \\ 55 & 1 & 0 & -2.164414 & -6.783951 & 0.578669 \\ 56 & 6 & 0 & -1.257833 & -6.350652 & 2.537677 \\ 57 & 1 & 0 & -1.242845 & -8.130080 & 1.238821 \\ 58 & 1 & 0 & -2.162979 & -5.750228 & 2.673012 \\ 59 & 6 & 0 & 0.000000 & -5.447323 & 2.687080 \\ 60 & 1 & 0 & -1.248725 & -7.098009 & 3.338245 \\ 61 & 1 & 0 & 0.000000 & -4.936340 & 3.654460 \\ 62 & 1 & 0 & 1.242845 & -8.130080 & 1.238821 \\ 63 & 6 & 0 & 1.257833 & -6.350652 & 2.537677 \\ 64 & 6 & 0 & 0.000000 & 1.277211 & -0.336973 \\ 65 & 6 & 0 & 0.000000 & 3.088176 & 1.422858 \\ & & & & & \end{array}$

$\mathrm{HF}=-2356.8230897$ hartree

Table S17. Cartesian Coordinates from the Optimized Structure of $1(3 \mathrm{~T})^{2+}$ (transoid-transoid) at B3LYP/6-31G(d)

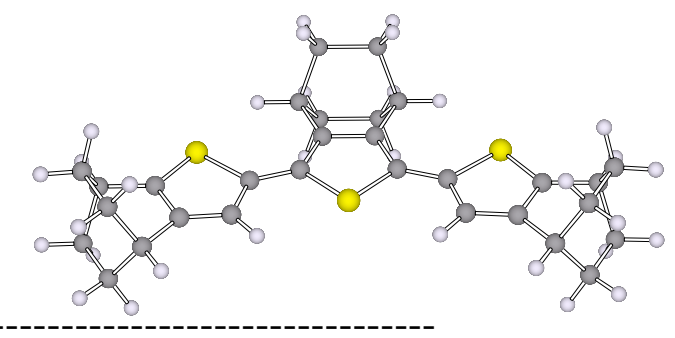

Center Atomic Atomic Coordinates (Angstroms)

Number Number Type $\quad$ X $\quad$ Y $\quad$ Z

$\begin{array}{llllll}1 & 6 & 0 & 1.265934 & 6.996740 & 1.118356 \\ 2 & 6 & 0 & 0.000000 & -0.691479 & -1.626390 \\ 3 & 6 & 0 & 0.000000 & 0.691479 & -1.626390 \\ 4 & 1 & 0 & 0.000000 & 4.930956 & 3.662879\end{array}$




$\begin{array}{cccccc}5 & 1 & 0 & 2.170863 & -6.740917 & 0.559864 \\ 6 & 1 & 0 & 2.170863 & 6.740917 & 0.559864 \\ 7 & 16 & 0 & 0.000000 & 3.995159 & -1.028905 \\ 8 & 6 & 0 & 0.000000 & 6.567591 & 0.297331 \\ 9 & 1 & 0 & 1.235350 & 8.088057 & 1.194306 \\ 10 & 6 & 0 & -1.265934 & 6.996740 & 1.118356 \\ 11 & 1 & 0 & 0.000000 & 7.017976 & -0.697499 \\ 12 & 1 & 0 & -1.235350 & 8.088057 & 1.194306 \\ 13 & 6 & 0 & -1.261190 & 6.329625 & 2.525169 \\ 14 & 1 & 0 & -2.170863 & 6.740917 & 0.559864 \\ 15 & 6 & 0 & 0.000000 & 4.454057 & 1.552292 \\ 16 & 1 & 0 & -2.166747 & 5.733310 & 2.672180 \\ 17 & 1 & 0 & -1.249157 & 7.087448 & 3.314135 \\ 18 & 1 & 0 & 2.166747 & 5.733310 & 2.672180 \\ 19 & 6 & 0 & 0.000000 & -4.454057 & 1.552292 \\ 20 & 6 & 0 & 1.261190 & 6.329625 & 2.525169 \\ 21 & 6 & 0 & 0.000000 & -2.603501 & 0.099196 \\ 22 & 6 & 0 & 0.000000 & 2.603501 & 0.099196 \\ 23 & 1 & 0 & 1.249157 & -7.087448 & 3.314135 \\ 24 & 1 & 0 & 0.000000 & 2.410274 & 2.302364 \\ 25 & 6 & 0 & -1.263972 & 0.775472 & -3.744443 \\ 26 & 6 & 0 & 0.000000 & -1.277333 & -0.308509 \\ 27 & 1 & 0 & -1.238319 & 1.169829 & -4.764927 \\ 28 & 6 & 0 & 0.000000 & -3.081656 & 1.449467 \\ 29 & 6 & 0 & 0.000000 & 1.304833 & -2.998908 \\ 30 & 16 & 0 & 0.000000 & 0.000000 & 0.922645 \\ 31 & 1 & 0 & 0.000000 & -2.394409 & -2.994541 \\ 32 & 6 & 0 & 0.000000 & 5.088747 & 0.278624 \\ 33 & 1 & 0 & 0.000000 & 2.394409 & -2.994541 \\ 34 & 6 & 0 & 1.263972 & 0.775472 & -3.744443 \\ 35 & 1 & 0 & -2.168217 & 1.169903 & -3.271464 \\ 36 & 1 & 0 & 1.238319 & 1.169829 & -4.764927 \\ 37 & 6 & 0 & 1.263972 & -0.775472 & -3.744443 \\ 38 & 1 & 0 & 2.168217 & 1.169903 & -3.271464 \\ 39 & 6 & 0 & 0.000000 & -1.304833 & -2.998908 \\ 40 & 1 & 0 & 2.168217 & -1.169903 & -3.271464 \\ 41 & 1 & 0 & 1.238319 & -1.169829 & -4.764927 \\ 42 & 6 & 0 & -1.263972 & -0.775472 & -3.744443 \\ 43 & 1 & 0 & -2.168217 & -1.169903 & -3.271464 \\ & & & & & \end{array}$




\begin{tabular}{|c|c|c|c|c|c|}
\hline 44 & 1 & 0 & -1.238319 & -1.169829 & -4.764927 \\
\hline 45 & 6 & 0 & 0.000000 & -6.567591 & 0.297331 \\
\hline 46 & 1 & 0 & 1.249157 & 7.087448 & 3.314135 \\
\hline 47 & 16 & 0 & 0.000000 & -3.995159 & -1.028905 \\
\hline 48 & 6 & 0 & 0.000000 & -5.088747 & 0.278624 \\
\hline 49 & 1 & 0 & 2.166747 & -5.733310 & 2.672180 \\
\hline 50 & 6 & 0 & 0.000000 & 5.433107 & 2.692056 \\
\hline 51 & 1 & 0 & 0.000000 & -2.410274 & 2.302364 \\
\hline 52 & 1 & 0 & 0.000000 & -7.017976 & -0.697499 \\
\hline 53 & 6 & 0 & -1.265934 & -6.996740 & 1.118356 \\
\hline 54 & 6 & 0 & 1.265934 & -6.996740 & 1.118356 \\
\hline 55 & 1 & 0 & -2.170863 & -6.740917 & 0.559864 \\
\hline 56 & 6 & 0 & -1.261190 & -6.329625 & 2.525169 \\
\hline 57 & 1 & 0 & -1.235350 & -8.088057 & 1.194306 \\
\hline 58 & 1 & 0 & -2.166747 & -5.733310 & 2.672180 \\
\hline 59 & 6 & 0 & 0.000000 & -5.433107 & 2.692056 \\
\hline 60 & 1 & 0 & -1.249157 & -7.087448 & 3.314135 \\
\hline 61 & 1 & 0 & 0.000000 & -4.930956 & 3.662879 \\
\hline 62 & 1 & 0 & 1.235350 & -8.088057 & 1.194306 \\
\hline 63 & 6 & 0 & 1.261190 & -6.329625 & 2.525169 \\
\hline 64 & 6 & 0 & 0.000000 & 1.277333 & -0.308509 \\
\hline 65 & 6 & 0 & 0.000000 & 3.081656 & 1.449467 \\
\hline
\end{tabular}

$\mathrm{HF}=-2356.48902$ hartree

Table S18. Cartesian Coordinates from the Optimized Structure of $1(3 \mathrm{~T})^{2+}$ (cisoid-transoid) at B3LYP/6$31 \mathrm{G}(\mathrm{d})$

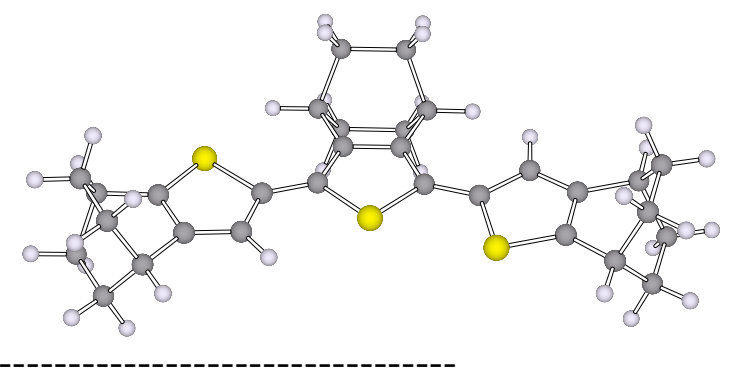

Center Atomic Atomic Coordinates (Angstroms)

Number Number Type $\quad$ X $\quad$ Y $\quad$ Z 


\begin{tabular}{|c|c|c|c|c|c|}
\hline 1 & 6 & 0 & 6.641743 & -2.177430 & 1.225946 \\
\hline 2 & 6 & 0 & -0.665061 & 1.638725 & -0.008957 \\
\hline 3 & 6 & 0 & 0.718332 & 1.615849 & -0.013639 \\
\hline 4 & 1 & 0 & 6.619119 & 1.215322 & 0.316219 \\
\hline 5 & 1 & 0 & -6.837248 & -0.344974 & -2.063462 \\
\hline 6 & 1 & 0 & 6.063533 & -2.561150 & 2.071252 \\
\hline 7 & 16 & 0 & 3.009264 & -1.878906 & -0.169439 \\
\hline 8 & 6 & 0 & 5.853349 & -2.422831 & -0.108931 \\
\hline 9 & 1 & 0 & 7.554678 & -2.777926 & 1.164442 \\
\hline 10 & 6 & 0 & 6.747782 & -1.906629 & -1.289572 \\
\hline 11 & 1 & 0 & 5.600498 & -3.477900 & -0.232636 \\
\hline 12 & 1 & 0 & 7.651806 & -2.523427 & -1.292248 \\
\hline 13 & 6 & 0 & 7.089823 & -0.399257 & -1.102056 \\
\hline 14 & 1 & 0 & 6.238019 & -2.083321 & -2.240976 \\
\hline 15 & 6 & 0 & 4.952003 & -0.145304 & 0.090824 \\
\hline 16 & 1 & 0 & 6.759272 & 0.181609 & -1.968356 \\
\hline 17 & 1 & 0 & 8.170875 & -0.254344 & -1.016792 \\
\hline 18 & 1 & 0 & 6.538123 & -0.284639 & 2.334043 \\
\hline 19 & 6 & 0 & -4.477579 & -1.482995 & -0.036098 \\
\hline 20 & 6 & 0 & 6.968188 & -0.665465 & 1.402733 \\
\hline 21 & 6 & 0 & -2.608230 & -0.054395 & -0.020018 \\
\hline 22 & 6 & 0 & 2.603907 & -0.135835 & -0.021825 \\
\hline 23 & 1 & 0 & -7.172978 & -3.143146 & -1.291768 \\
\hline 24 & 1 & 0 & 3.816864 & 1.697852 & 0.234594 \\
\hline 25 & 6 & 0 & 0.841522 & 3.749250 & 1.225771 \\
\hline 26 & 6 & 0 & -1.276208 & 0.333091 & -0.028863 \\
\hline 27 & 1 & 0 & 1.247273 & 4.764800 & 1.183590 \\
\hline 28 & 6 & 0 & -3.104786 & -1.397015 & -0.074568 \\
\hline 29 & 6 & 0 & 1.348159 & 2.981536 & -0.033363 \\
\hline 30 & 16 & 0 & -0.021653 & -0.919144 & -0.058955 \\
\hline 31 & 1 & 0 & -2.349222 & 3.028689 & -0.002755 \\
\hline 32 & 6 & 0 & 4.674112 & -1.531831 & -0.062864 \\
\hline 33 & 1 & 0 & 2.435759 & 2.963571 & -0.052345 \\
\hline 34 & 6 & 0 & 0.816767 & 3.720142 & -1.301334 \\
\hline 35 & 1 & 0 & 1.238397 & 3.283287 & 2.132698 \\
\hline 36 & 1 & 0 & 1.229272 & 4.733786 & -1.292727 \\
\hline 37 & 6 & 0 & -0.733047 & 3.747547 & -1.286229 \\
\hline 38 & 1 & 0 & 1.194087 & 3.228692 & -2.203061 \\
\hline 39 & 6 & 0 & -1.259595 & 3.019645 & -0.011091 \\
\hline
\end{tabular}




\begin{tabular}{|c|c|c|c|c|c|}
\hline 40 & 1 & 0 & -1.145224 & 3.275065 & -2.182733 \\
\hline 41 & 1 & 0 & -1.109697 & 4.774776 & -1.263439 \\
\hline 42 & 6 & 0 & -0.709532 & 3.767801 & 1.241412 \\
\hline 43 & 1 & 0 & -1.099693 & 3.305395 & 2.152951 \\
\hline 44 & 1 & 0 & -1.091866 & 4.792770 & 1.213450 \\
\hline 45 & 6 & 0 & -6.572281 & -0.205260 & 0.109940 \\
\hline 46 & 1 & 0 & 8.048849 & -0.506461 & 1.464196 \\
\hline 47 & 16 & 0 & -3.983165 & 1.089343 & 0.083149 \\
\hline 48 & 6 & 0 & -5.094370 & -0.203418 & 0.054540 \\
\hline 49 & 1 & 0 & -5.843759 & -2.458540 & -2.215220 \\
\hline 50 & 6 & 0 & 6.422265 & 0.147180 & 0.193292 \\
\hline 51 & 1 & 0 & -2.445511 & -2.256607 & -0.143609 \\
\hline 52 & 1 & 0 & -7.009211 & 0.792970 & 0.181675 \\
\hline 53 & 6 & 0 & -6.962273 & -1.090125 & 1.345428 \\
\hline 54 & 6 & 0 & -7.060567 & -0.951201 & -1.180993 \\
\hline 55 & 1 & 0 & -6.658427 & -0.587528 & 2.268070 \\
\hline 56 & 6 & 0 & -6.321053 & -2.504372 & 1.233519 \\
\hline 57 & 1 & 0 & -8.055062 & -1.147594 & 1.357952 \\
\hline 58 & 1 & 0 & -5.697629 & -2.715071 & 2.107689 \\
\hline 59 & 6 & 0 & -5.470884 & -2.609925 & -0.065424 \\
\hline 60 & 1 & 0 & -7.092795 & -3.279136 & 1.200755 \\
\hline 61 & 1 & 0 & -4.981847 & -3.584695 & -0.137891 \\
\hline 62 & 1 & 0 & -8.150352 & -1.020808 & -1.108681 \\
\hline 63 & 6 & 0 & -6.407886 & -2.361223 & -1.282720 \\
\hline 64 & 6 & 0 & 1.279539 & 0.284188 & -0.030193 \\
\hline 65 & 6 & 0 & 3.809687 & 0.624222 & 0.110172 \\
\hline
\end{tabular}

$\mathrm{HF}=-2356.4867067$ hartree

Table S19. Cartesian Coordinates from the Optimized Structure of $\mathbf{1}(3 \mathrm{~T})^{2+}$ (cisoid-cisoid) at B3LYP/6$31 \mathrm{G}(\mathrm{d})$

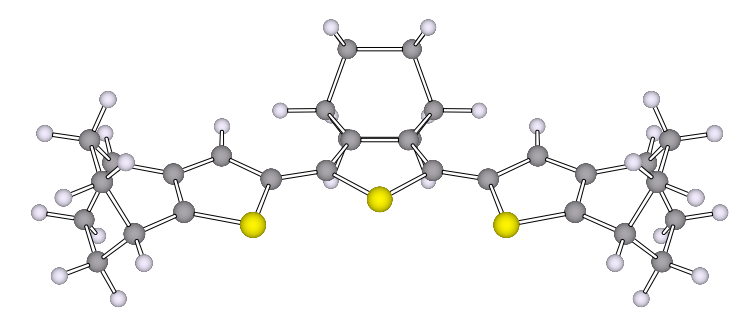


Center Atomic Atomic Coordinates (Angstroms)

Number Number Type $\quad$ X $\quad$ Y $\quad$ Z

$\begin{array}{cccccc}1 & 6 & 0 & 6.675049 & -2.102758 & 1.216903 \\ 2 & 6 & 0 & -0.692044 & 1.620206 & -0.000065 \\ 3 & 6 & 0 & 0.692061 & 1.620183 & 0.000116 \\ 4 & 16 & 0 & 3.045125 & -1.829528 & -0.192671 \\ 5 & 6 & 0 & 5.897156 & -2.327387 & -0.127766 \\ 6 & 6 & 0 & 6.788379 & -1.766463 & -1.290205 \\ 7 & 6 & 0 & 7.104379 & -0.258857 & -1.062240 \\ 8 & 6 & 0 & 4.956750 & -0.070637 & 0.124313 \\ 9 & 6 & 0 & -4.956767 & -0.070613 & 0.124199 \\ 10 & 6 & 0 & 6.974822 & -0.590504 & 1.434141 \\ 11 & 6 & 0 & -2.610083 & -0.097074 & -0.007191 \\ 12 & 6 & 0 & 2.610055 & -0.097126 & -0.006937 \\ 13 & 6 & 0 & 0.775779 & 3.776027 & 1.204958 \\ 14 & 6 & 0 & -1.278850 & 0.300264 & -0.016763 \\ 15 & 6 & 0 & -3.801802 & 0.679451 & 0.153417 \\ 16 & 6 & 0 & 1.303394 & 2.994004 & -0.035902 \\ 17 & 16 & 0 & -0.000030 & -0.925259 & -0.046877 \\ 18 & 6 & 0 & 4.702915 & -1.457697 & -0.065708 \\ 19 & 6 & 0 & 0.774729 & 3.702971 & -1.321374 \\ 20 & 6 & 0 & -0.774655 & 3.703876 & -1.320818 \\ 21 & 6 & 0 & -1.303339 & 2.994049 & -0.035817 \\ 22 & 6 & 0 & -0.775702 & 3.775353 & 1.205479 \\ 23 & 6 & 0 & -5.897174 & -2.327353 & -0.127945 \\ 24 & 16 & 0 & -3.045137 & -1.829517 & -0.192653 \\ 25 & 6 & 0 & -4.702922 & -1.457708 & -0.065557 \\ 26 & 6 & 0 & 6.421247 & 0.243458 & 0.243031 \\ 27 & 6 & 0 & -6.787670 & -1.766047 & -1.290777 \\ 28 & 6 & 0 & -6.675901 & -2.102987 & 1.216268 \\ 29 & 6 & 0 & -7.105035 & -0.258910 & -1.061641 \\ 30 & 6 & 0 & -6.421241 & 0.243438 & 0.243284 \\ 31 & 6 & 0 & -6.974063 & -0.590581 & 1.434690 \\ 32 & 6 & 0 & 1.278828 & 0.300231 & -0.016588 \\ 33 & 6 & 0 & 3.801769 & 0.679398 & 0.153755 \\ 34 & 1 & 0 & 6.099634 & -2.517875 & 2.049173 \\ 35 & 1 & 0 & 7.598357 & -2.685955 & 1.144508\end{array}$




$\begin{array}{llllll}36 & 1 & 0 & 5.662695 & -3.383043 & -0.279437 \\ 37 & 1 & 0 & 7.702513 & -2.368001 & -1.303733 \\ 38 & 1 & 0 & 6.286156 & -1.927008 & -2.248438 \\ 39 & 1 & 0 & 6.768909 & 0.338407 & -1.915402 \\ 40 & 1 & 0 & 8.182417 & -0.098459 & -0.967089 \\ 41 & 1 & 0 & 6.533743 & -0.241125 & 2.372578 \\ 42 & 1 & 0 & 8.052309 & -0.414959 & 1.505362 \\ 43 & 1 & 0 & 1.169277 & 4.795803 & 1.150808 \\ 44 & 1 & 0 & 1.168995 & 3.329548 & 2.123213 \\ 45 & 1 & 0 & -3.789706 & 1.749246 & 0.307899 \\ 46 & 1 & 0 & 2.391333 & 2.988911 & -0.045640 \\ 47 & 1 & 0 & 1.170237 & 4.723386 & -1.325381 \\ 48 & 1 & 0 & 1.169391 & 3.203052 & -2.210914 \\ 49 & 1 & 0 & -1.170592 & 3.205741 & -2.210783 \\ 50 & 1 & 0 & -1.168891 & 4.724791 & -1.323055 \\ 51 & 1 & 0 & -2.391277 & 2.988987 & -0.045593 \\ 52 & 1 & 0 & -1.167894 & 3.327465 & 2.123491 \\ 53 & 1 & 0 & -1.170155 & 4.794832 & 1.152723 \\ 54 & 1 & 0 & -5.662718 & -3.382994 & -0.279728 \\ 55 & 1 & 0 & 6.599575 & 1.311286 & 0.393807 \\ 56 & 1 & 0 & -6.284242 & -1.925227 & -2.248611 \\ 57 & 1 & 0 & -7.701310 & -2.368294 & -1.305924 \\ 58 & 1 & 0 & -6.101808 & -2.519630 & 2.048681 \\ 59 & 1 & 0 & -7.599870 & -2.684954 & 1.142415 \\ 60 & 1 & 0 & -6.771138 & 0.339187 & -1.914828 \\ 61 & 1 & 0 & -8.183148 & -0.099733 & -0.965254 \\ 62 & 1 & 0 & -6.599561 & 1.311253 & 0.394160 \\ 63 & 1 & 0 & -8.051287 & -0.414128 & 1.507571 \\ ----------------------------------------------------------\end{array}$

$H F=-2356.4842613$ hartree 
Table S20. Cartesian Coordinates from the Optimized Structure of $\mathbf{1}(4 \mathrm{~T})^{2+}$ at B3LYP/6-31G(d)

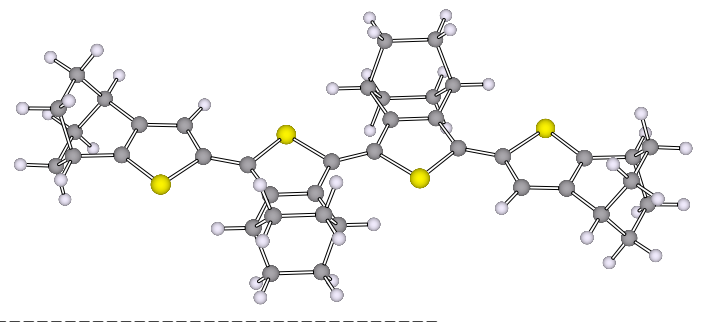

\begin{tabular}{|c|c|c|c|c|c|}
\hline \multirow{2}{*}{$\begin{array}{l}\text { Center } \\
\text { Number }\end{array}$} & Atomic & \multicolumn{2}{|c|}{ Atomic } & \multicolumn{2}{|c|}{ Coordinates (Angstroms } \\
\hline & Numbe & & Type & $X$ & $\mathrm{Z}$ \\
\hline 1 & 16 & 0 & 2.064777 & -0.818923 & -0.000114 \\
\hline 2 & 16 & 0 & 5.839502 & 1.512280 & -0.000030 \\
\hline 3 & 6 & 0 & 6.563252 & -0.994376 & 0.000092 \\
\hline 4 & 6 & 0 & 0.371533 & 2.942292 & 0.000238 \\
\hline 5 & 1 & 0 & -0.712171 & 2.844700 & 0.000517 \\
\hline 6 & 6 & 0 & 4.573285 & 0.255691 & 0.000028 \\
\hline 7 & 6 & 0 & 5.178947 & -1.029482 & 0.000135 \\
\hline 8 & 1 & 0 & 4.597627 & -1.945981 & 0.000214 \\
\hline 9 & 6 & 0 & 2.485121 & 1.767790 & -0.000170 \\
\hline 10 & 6 & 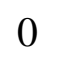 & & & -0.000045 \\
\hline 11 & 6 & 0 & 0.826942 & 3.732691 & -1.263550 \\
\hline 12 & 1 & 0 & 0.333323 & 4.709696 & -1.242962 \\
\hline 13 & 1 & & 0.481677 & 3.219836 & -2.166467 \\
\hline 14 & 6 & 0 & 2.963385 & 3.196377 & -0.000206 \\
\hline 15 & 1 & 0 & 51 & 3.291029 & -0.000481 \\
\hline 16 & 6 & 0 & 1.104352 & 1.625162 & -0.000079 \\
\hline 17 & 6 & 0 & 2.371031 & 3.886074 & 1.262756 \\
\hline 18 & 1 & 0 & 2.661864 & 4.941202 & 1.242959 \\
\hline 19 & 1 & 0 & 2.810248 & 3.450657 & 2.165388 \\
\hline 20 & 6 & 0 & 0.827499 & 3.732270 & 1.264157 \\
\hline 21 & 1 & 0 & 0.482891 & 3.218933 & 2.167059 \\
\hline 22 & 1 & 0 & 0.333786 & 4.709245 & 1.244276 \\
\hline 23 & 6 & 0 & 3.199649 & 0.530906 & -0.000041 \\
\hline 24 & 6 & 0 & 8.527049 & -1.785525 & 1.259647 \\
\hline 25 & 1 & 0 & 9.357951 & -2.497691 & 1.248570 \\
\hline 26 & 1 & 0 & 7.946890 & -1.988196 & 2.165141 \\
\hline 27 & 6 & 0 & 8.546530 & 0.449190 & -0.000024 \\
\hline 28 & 1 & 0 & 8.894721 & 1.484623 & -0.000062 \\
\hline 29 & 6 & 0 & 8.526860 & -1.785551 & -1.259700 \\
\hline
\end{tabular}




\begin{tabular}{|c|c|c|c|c|c|}
\hline 30 & 1 & 0 & 9.357629 & -2.497881 & -1.248907 \\
\hline 31 & 1 & 0 & 7.946460 & -1.987987 & -2.165095 \\
\hline 32 & 6 & 0 & 7.065973 & 0.322452 & -0.000016 \\
\hline 33 & 6 & 0 & 2.370416 & 3.886308 & -1.262806 \\
\hline 34 & 1 & 0 & 2.661302 & 4.941413 & -1.242982 \\
\hline 35 & 1 & 0 & 2.809279 & 3.451018 & -2.165672 \\
\hline 36 & 6 & 0 & 7.648110 & -2.035687 & 0.000059 \\
\hline 37 & 1 & 0 & 7.243433 & -3.051461 & 0.000096 \\
\hline 38 & 6 & 0 & 9.055846 & -0.320459 & -1.263360 \\
\hline 39 & 1 & 0 & 8.744530 & 0.209559 & -2.168282 \\
\hline 40 & 1 & 0 & 10.149887 & -0.294551 & -1.238709 \\
\hline 41 & 6 & 0 & 9.055784 & -0.320333 & 1.263401 \\
\hline 42 & 1 & 0 & 10.149824 & -0.294217 & 1.238942 \\
\hline 43 & 1 & 0 & 8.744207 & 0.209639 & 2.168261 \\
\hline 44 & 16 & 0 & -2.064878 & 0.819199 & -0.000337 \\
\hline 45 & 16 & 0 & -5.839375 & -1.512397 & -0.000161 \\
\hline 46 & 6 & 0 & -6.563430 & 0.994172 & -0.000102 \\
\hline 47 & 6 & 0 & -0.371370 & -2.941850 & -0.000106 \\
\hline 48 & 1 & 0 & 0.712326 & -2.844141 & -0.000354 \\
\hline 49 & 6 & 0 & -4.573313 & -0.255654 & -0.000250 \\
\hline 50 & 6 & 0 & -5.179129 & 1.029448 & -0.000243 \\
\hline 51 & 1 & 0 & -4.597924 & 1.946019 & -0.000263 \\
\hline 52 & 6 & 0 & -2.485036 & -1.767558 & 0.000018 \\
\hline 53 & 6 & 0 & -0.647200 & -0.257342 & -0.000205 \\
\hline 54 & 6 & 0 & -0.826812 & -3.732004 & 1.263850 \\
\hline 55 & 1 & 0 & -0.333042 & -4.708936 & 1.243518 \\
\hline 56 & 1 & 0 & -0.481733 & -3.218912 & 2.166704 \\
\hline 57 & 6 & 0 & -2.963201 & -3.196201 & 0.000270 \\
\hline 58 & 1 & 0 & -4.048557 & -3.290977 & 0.000501 \\
\hline 59 & 6 & 0 & -1.104285 & -1.624798 & -0.000077 \\
\hline 60 & 6 & 0 & -2.370715 & -3.886142 & -1.262483 \\
\hline 61 & 1 & 0 & -2.661267 & -4.941346 & -1.242411 \\
\hline 62 & 1 & 0 & -2.810080 & -3.451056 & -2.165202 \\
\hline 63 & 6 & 0 & -0.827211 & -3.732076 & -1.263912 \\
\hline 64 & 1 & 0 & -0.482588 & -3.218887 & -2.166892 \\
\hline 65 & 1 & 0 & -0.333320 & -4.708954 & -1.243784 \\
\hline 66 & 6 & 0 & -3.199649 & -0.530725 & -0.000230 \\
\hline 67 & 6 & 0 & -8.527460 & 1.785211 & -1.259390 \\
\hline 68 & 1 & 0 & -9.358476 & 2.497242 & -1.248116 \\
\hline
\end{tabular}




$\begin{array}{lllrrr}69 & 1 & 0 & -7.947444 & 1.988102 & -2.164926 \\ 70 & 6 & 0 & -8.546533 & -0.449638 & 0.000041 \\ 71 & 1 & 0 & -8.894597 & -1.485114 & 0.000012 \\ 72 & 6 & 0 & -8.527005 & 1.784971 & 1.259955 \\ 73 & 1 & 0 & -9.357837 & 2.497230 & 1.249347 \\ 74 & 1 & 0 & -7.946519 & 1.987337 & 2.165311 \\ 75 & 6 & 0 & -7.065992 & -0.322719 & -0.000057 \\ 76 & 6 & 0 & -2.370265 & -3.885860 & 1.263013 \\ 77 & 1 & 0 & -2.661028 & -4.941005 & 1.243362 \\ 78 & 1 & 0 & -2.809287 & -3.450428 & 2.165733 \\ 79 & 6 & 0 & -7.648417 & 2.035347 & 0.000131 \\ 80 & 1 & 0 & -7.243864 & 3.051171 & 0.000162 \\ 81 & 6 & 0 & -9.055862 & 0.319833 & 1.263477 \\ 82 & 1 & 0 & -8.744466 & -0.210256 & 2.168329 \\ 83 & 1 & 0 & -10.149902 & 0.293831 & 1.238863 \\ 84 & 6 & 0 & -9.055962 & 0.319934 & -1.263285 \\ 85 & 1 & 0 & -10.149996 & 0.293637 & -1.238799 \\ 86 & 1 & 0 & -8.744324 & -0.209893 & -2.168210 \\ & & & & & \end{array}$

$\mathrm{HF}=-3141.8053372$ hartree 
Table S21. Cartesian Coordinates from the Optimized Structure of bithiophene dication $\left(\mathrm{C}_{b}-\mathrm{C}_{\mathrm{a}}-\mathrm{C}_{\mathrm{a}}-\mathrm{C}_{\mathrm{b}}=\right.$ $\left.0^{\circ}\right)$ at $\mathrm{B} 3 \mathrm{LYP} / 6-31 \mathrm{G}(\mathrm{d})$

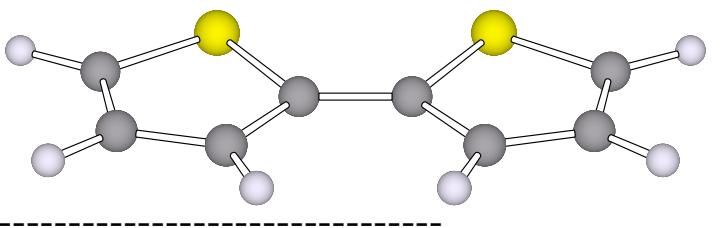

\begin{tabular}{|c|c|c|c|c|c|}
\hline \multirow{2}{*}{$\begin{array}{l}\text { Center } \\
\text { Number }\end{array}$} & \multirow{2}{*}{\multicolumn{2}{|c|}{$\begin{array}{l}\text { Atomic } \\
\text { Number }\end{array}$}} & Atomic & \multicolumn{2}{|c|}{ Coordinates (Angstroms } \\
\hline & & & Type & $X$ & Z \\
\hline 1 & 16 & 0 & 1.674389 & -1.206175 & 0.000000 \\
\hline 2 & 6 & 0 & 3.084580 & -0.285220 & 0.000000 \\
\hline 3 & 6 & 0 & 2.888357 & 1.131260 & 0.000001 \\
\hline 4 & 6 & 0 & 1.563180 & 1.469783 & 0.000000 \\
\hline 5 & 6 & 0 & & 0.311605 & -0.000001 \\
\hline 6 & 6 & 0 & -0.684048 & 0.311581 & -0.000001 \\
\hline 7 & 6 & 0 & -1.563263 & 1.469823 & 0.000000 \\
\hline 8 & 6 & 0 & -2.888406 & 1.131199 & 0.000001 \\
\hline 9 & 6 & 0 & -3.084535 & -0.285296 & 0.000001 \\
\hline 10 & 16 & 0 & -1.674303 & -1.206201 & 0.000000 \\
\hline 11 & 1 & 0 & 4.051578 & -0.786729 & 0.000001 \\
\hline 12 & 1 & 0 & 3.717557 & 1.831716 & 0.000001 \\
\hline 13 & 1 & 0 & 1.194403 & 2.489899 & -0.000001 \\
\hline 14 & 1 & 0 & -1.194538 & 2.489948 & -0.000001 \\
\hline 15 & 1 & 0 & -3.717699 & 1.831552 & 0.000001 \\
\hline 16 & 1 & 0 & -4.051542 & -0.786777 & 0.000002 \\
\hline
\end{tabular}

$\mathrm{HF}=-1104.1079205$ hartree 
Table S22. Cartesian Coordinates from the Optimized Structure of bithiophene dication $\left(C_{b}-C_{a}-C_{a}-C_{b}=\right.$ $\left.10^{\circ}\right)$ at B3LYP/6-31G(d)

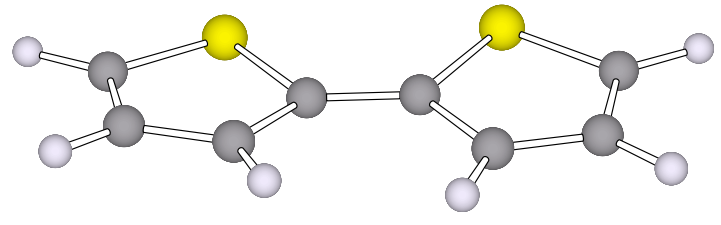

\begin{tabular}{|c|c|c|c|c|c|}
\hline \multirow{2}{*}{$\begin{array}{l}\text { Center } \\
\text { Number }\end{array}$} & \multirow{2}{*}{$\begin{array}{l}\text { Atomic } \\
\text { Number }\end{array}$} & \multicolumn{2}{|c|}{ Atomic } & \multicolumn{2}{|c|}{ Coordinates (Angstroms) } \\
\hline & & & Type & $\mathrm{X}$ & $\mathrm{Z}$ \\
\hline 1 & 16 & 0 & -1.672641 & -1.204412 & 0.069370 \\
\hline 2 & 6 & 0 & -3.083684 & -0.286572 & 0.006023 \\
\hline 3 & 6 & 0 & -2.888571 & 1.128233 & -0.063223 \\
\hline 4 & 6 & 0 & -1.563958 & 1.468889 & -0.050115 \\
\hline 5 & 6 & 0 & -0.683754 & 0.313548 & 0.021555 \\
\hline 6 & 6 & 0 & 0.683758 & 0.313673 & -0.023004 \\
\hline 7 & 6 & 0 & 1.563943 & 1.468919 & 0.050224 \\
\hline 8 & 6 & 0 & 2.888539 & 1.128220 & 0.063914 \\
\hline 9 & 6 & 0 & 3.083683 & -0.286601 & -0.005459 \\
\hline 10 & 16 & 0 & 1.672657 & -1.204392 & -0.069409 \\
\hline 11 & 1 & 0 & -4.050291 & -0.788530 & 0.023394 \\
\hline 12 & 1 & 0 & -3.717960 & 1.826035 & -0.121817 \\
\hline 13 & 1 & 0 & -1.195506 & 2.487946 & -0.100306 \\
\hline 14 & 1 & 0 & 1.195524 & 2.488058 & 0.098965 \\
\hline 15 & 1 & 0 & 3.717904 & 1.826023 & 0.122819 \\
\hline 16 & 1 & 0 & 4.050331 & -0.788516 & -0.021906 \\
\hline
\end{tabular}

$\mathrm{HF}=-1104.1074737$ hartree 
Table S23. Cartesian Coordinates from the Optimized Structure of bithiophene dication $\left(C_{b}-C_{a}-C_{a}-C_{b}=\right.$ $\left.20^{\circ}\right)$ at B3LYP/6-31G(d)

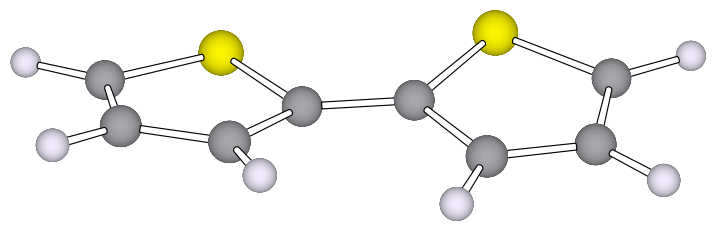

\begin{tabular}{|c|c|c|c|c|c|}
\hline \multirow{2}{*}{$\begin{array}{l}\text { Center } \\
\text { Number }\end{array}$} & \multirow{2}{*}{$\begin{array}{l}\text { Atomic } \\
\text { Number }\end{array}$} & \multicolumn{2}{|c|}{ Atomic } & \multicolumn{2}{|c|}{ Coordinates (Angstroms } \\
\hline & & & Type & $X$ & $\mathrm{Z}$ \\
\hline 1 & 16 & 0 & 1.667921 & -1.199148 & -0.138966 \\
\hline 2 & 6 & 0 & 3.081007 & -0.289997 & -0.011756 \\
\hline 3 & 6 & 0 & 2.888529 & 1.119631 & 0.126942 \\
\hline 4 & 6 & 0 & 1.565492 & 1.466051 & 0.100683 \\
\hline 5 & 6 & 0 & 0.683036 & 0.318850 & -0.044323 \\
\hline 6 & 6 & 0 & -0.682983 & 0.319113 & 0.044514 \\
\hline 7 & 6 & 0 & -1.565597 & 1.466082 & -0.101069 \\
\hline 8 & 6 & 0 & -2.888590 & 1.119532 & -0.126965 \\
\hline 9 & 6 & 0 & -3.080948 & -0.290162 & 0.011677 \\
\hline 10 & 16 & 0 & -1.667876 & -1.199113 & 0.139010 \\
\hline 11 & 1 & 0 & 4.046560 & -0.793128 & -0.046218 \\
\hline 12 & 1 & & 3.717950 & 1.810009 & 0.244178 \\
\hline 13 & 1 & 0 & 1.197730 & 2.481925 & 0.200025 \\
\hline 14 & 1 & 0 & -1.198036 & 2.482174 & -0.198934 \\
\hline 15 & 1 & 0 & -3.718095 & 1.809877 & -0.243790 \\
\hline 16 & 1 & 0 & -4.046520 & -0.793275 & 0.045819 \\
\hline
\end{tabular}

$\mathrm{HF}=-1104.1061077$ hartree 
Table S24. Cartesian Coordinates from the Optimized Structure of bithiophene dication $\left(\mathrm{C}_{b}-\mathrm{C}_{\mathrm{a}}-\mathrm{C}_{\mathrm{a}}-\mathrm{C}_{\mathrm{b}}=\right.$ $\left.30^{\circ}\right)$ at B3LYP/6-31G(d)

$\begin{array}{cccccc}1 & 16 & 0 & -1.658998 & -1.189927 & 0.209306 \\ 2 & 6 & 0 & -3.076201 & -0.296741 & 0.018357 \\ 3 & 6 & 0 & -2.889333 & 1.104177 & -0.191012 \\ 4 & 6 & 0 & -1.569356 & 1.461538 & -0.151730 \\ 5 & 6 & 0 & -0.681873 & 0.329103 & 0.066662 \\ 6 & 6 & 0 & 0.681782 & 0.329444 & -0.065912 \\ 7 & 6 & 0 & 1.569610 & 1.461469 & 0.152721 \\ 8 & 6 & 0 & 2.889579 & 1.103994 & 0.190179 \\ 9 & 6 & 0 & 3.076139 & -0.296980 & -0.019694 \\ 10 & 16 & 0 & 1.658801 & -1.189985 & -0.208962 \\ 11 & 1 & 0 & -4.039549 & -0.802640 & 0.070193 \\ 12 & 1 & 0 & -3.719319 & 1.781330 & -0.367056 \\ 13 & 1 & 0 & -1.204161 & 2.472770 & -0.298975 \\ 14 & 1 & 0 & 1.204788 & 2.472906 & 0.299513 \\ 15 & 1 & 0 & 3.719820 & 1.781053 & 0.365371 \\ 16 & 1 & 0 & 4.039483 & -0.802842 & -0.071977 \\ ------------------1\end{array}$

$\mathrm{HF}=-1104.1037504$ hartree 
Table S25. Cartesian Coordinates from the Optimized Structure of bithiophene dication $\left(\mathrm{C}_{b}-\mathrm{C}_{\mathrm{a}}-\mathrm{C}_{\mathrm{a}}-\mathrm{C}_{\mathrm{b}}=\right.$ $\left.40^{\circ}\right)$ at B3LYP/6-31G(d)

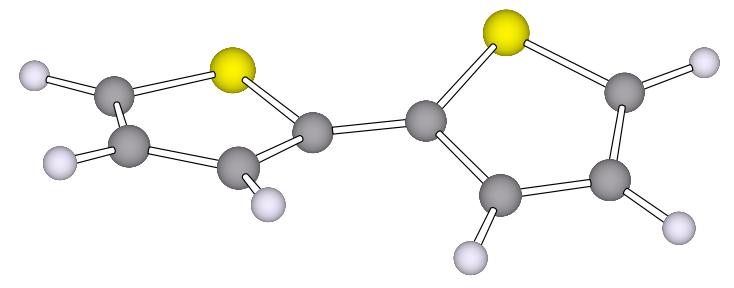

\begin{tabular}{ccccccc} 
Center & \multicolumn{2}{c}{ Atomic } & \multicolumn{2}{c}{ Atomic } & \multicolumn{3}{c}{ Coordinates (Angstroms) } \\
Number & \multicolumn{2}{c}{ Number } & Type & X & Y & Z \\
---------------------------------------------- \\
1 & 16 & 0 & -1.645270 & -1.176580 & 0.279894 \\
2 & 6 & 0 & -3.069203 & -0.307352 & 0.027294 \\
3 & 6 & 0 & -2.891587 & 1.081171 & -0.253741 \\
4 & 6 & 0 & -1.576304 & 1.455116 & -0.203907 \\
5 & 6 & 0 & -0.680424 & 0.344932 & 0.087656 \\
6 & 6 & 0 & 0.680244 & 0.345776 & -0.087492 \\
7 & 6 & 0 & 1.576674 & 1.455158 & 0.204755 \\
8 & 6 & 0 & 2.891836 & 1.080849 & 0.253427 \\
9 & 6 & 0 & 3.069038 & -0.307807 & -0.028122 \\
10 & 16 & 0 & 1.645076 & -1.176532 & -0.279721 \\
11 & 1 & 0 & -4.028899 & -0.817908 & 0.098197 \\
12 & 1 & 0 & -3.723059 & 1.739033 & -0.487008 \\
13 & 1 & 0 & -1.216385 & 2.460447 & -0.398986 \\
14 & 1 & 0 & 1.217399 & 2.461057 & 0.398128 \\
15 & 1 & 0 & 3.723627 & 1.738433 & 0.486321 \\
16 & 1 & 0 & 4.028784 & -0.818325 & -0.098635
\end{tabular}

$\mathrm{HF}=-1104.1003025$ hartree 
Table S26. Cartesian Coordinates from the Optimized Structure of bithiophene dication $\left(\mathrm{C}_{b}-\mathrm{C}_{\mathrm{a}}-\mathrm{C}_{\mathrm{a}}-\mathrm{C}_{\mathrm{b}}=\right.$ $\left.50^{\circ}\right)$ at $\mathrm{B} 3 \mathrm{LYP} / 6-31 \mathrm{G}(\mathrm{d})$

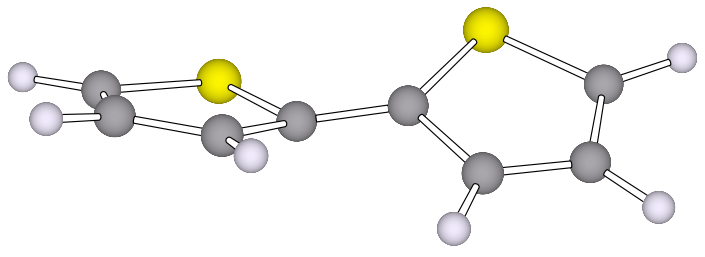

\begin{tabular}{|c|c|c|c|c|c|}
\hline \multirow{2}{*}{$\begin{array}{l}\text { Center } \\
\text { Numbes }\end{array}$} & \multirow{2}{*}{$\begin{array}{l}\text { Atomic } \\
\text { Numbe }\end{array}$} & \multicolumn{2}{|c|}{ Atomic } & \multicolumn{2}{|c|}{ Coordinates (Angstroms } \\
\hline & & & Type & $\mathrm{X}$ & $\mathrm{Z}$ \\
\hline 1 & 16 & 0 & -1.626531 & -1.158158 & 0.352252 \\
\hline 2 & 6 & 0 & -3.059779 & -0.322117 & 0.038444 \\
\hline 3 & 6 & 0 & -2.895343 & 1.049600 & -0.317142 \\
\hline 4 & 6 & 0 & -1.586880 & 1.446486 & -0.258840 \\
\hline 5 & 6 & 0 & -0.678857 & 0.367319 & 0.107740 \\
\hline 6 & 6 & 0 & 0.678788 & 0.367384 & -0.106195 \\
\hline 7 & 6 & 0 & 1.587311 & 1.446262 & 0.259707 \\
\hline 8 & 6 & 0 & 2.895838 & 1.049341 & 0.315899 \\
\hline 9 & 6 & 0 & 3.059756 & -0.322321 & -0.040472 \\
\hline 10 & 16 & 0 & 1.626114 & -1.158385 & -0.351670 \\
\hline 11 & 1 & 0 & -4.014196 & -0.839163 & 0.130706 \\
\hline 12 & 1 & 0 & -3.729276 & 1.681283 & -0.607393 \\
\hline 13 & 1 & 0 & -1.235484 & 2.444733 & -0.501714 \\
\hline 14 & 1 & 0 & 1.236325 & 2.444463 & 0.503372 \\
\hline 15 & 1 & 0 & 3.730199 & 1.680843 & 0.605317 \\
\hline 16 & 1 & 0 & 4.014095 & -0.839204 & -0.134446 \\
\hline
\end{tabular}

$\mathrm{HF}=-1104.095663$ hartree 
Table S27. Cartesian Coordinates from the Optimized Structure of bithiophene dication $\left(\mathrm{C}_{b}-\mathrm{C}_{\mathrm{a}}-\mathrm{C}_{\mathrm{a}}-\mathrm{C}_{\mathrm{b}}=\right.$ $\left.60^{\circ}\right)$ at $\mathrm{B} 3 \mathrm{LYP} / 6-31 \mathrm{G}(\mathrm{d})$

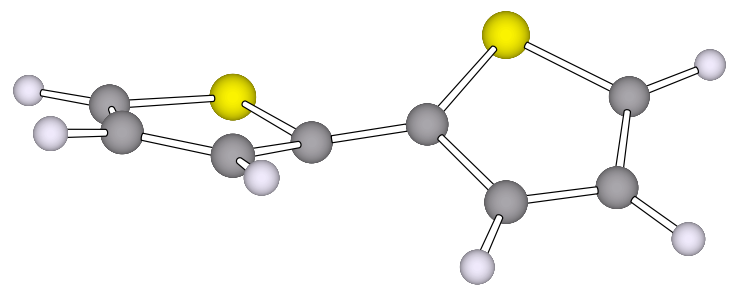

$\begin{array}{cccccc}1 & 16 & 0 & -1.601787 & -1.134415 & 0.424808 \\ 2 & 6 & 0 & -3.047827 & -0.341536 & 0.054439 \\ 3 & 6 & 0 & -2.901782 & 1.008464 & -0.378307 \\ 4 & 6 & 0 & -1.602367 & 1.434874 & -0.316795 \\ 5 & 6 & 0 & -0.677851 & 0.396967 & 0.124362 \\ 6 & 6 & 0 & 0.677766 & 0.396956 & -0.122351 \\ 7 & 6 & 0 & 1.602984 & 1.434517 & 0.317809 \\ 8 & 6 & 0 & 2.902527 & 1.008168 & 0.376560 \\ 9 & 6 & 0 & 3.047789 & -0.341811 & -0.056875 \\ 10 & 16 & 0 & 1.601177 & -1.134763 & -0.424081 \\ 11 & 1 & 0 & -3.994823 & -0.866862 & 0.172965 \\ 12 & 1 & 0 & -3.739885 & 1.606578 & -0.722923 \\ 13 & 1 & 0 & -1.264123 & 2.424539 & -0.609597 \\ 14 & 1 & 0 & 1.265278 & 2.423959 & 0.611992 \\ 15 & 1 & 0 & 3.741251 & 1.606056 & 0.720053 \\ 16 & 1 & 0 & 3.994623 & -0.867032 & -0.177174 \\ ---------------------------------------------------------\end{array}$

$\mathrm{HF}=-1104.0897515$ hartree 
Table S28. Cartesian Coordinates from the Optimized Structure of bithiophene dication $\left(\mathrm{C}_{b}-\mathrm{C}_{\mathrm{a}}-\mathrm{C}_{\mathrm{a}}-\mathrm{C}_{\mathrm{b}}=\right.$ $\left.70^{\circ}\right)$ at $\mathrm{B} 3 \mathrm{LYP} / 6-31 \mathrm{G}(\mathrm{d})$

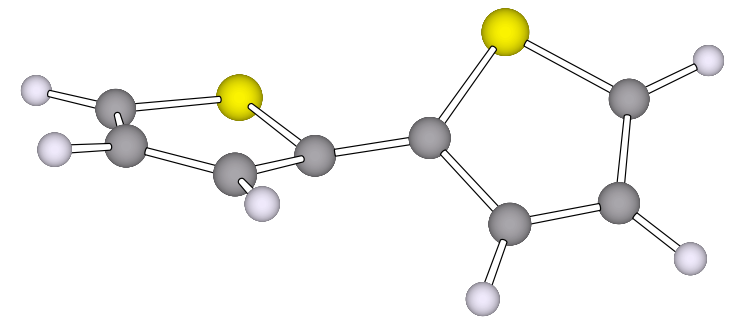

\begin{tabular}{|c|c|c|c|c|c|}
\hline \multirow{2}{*}{$\begin{array}{l}\text { Center } \\
\text { Numbe }\end{array}$} & \multirow{2}{*}{$\begin{array}{l}\text { Atomic } \\
\text { Number }\end{array}$} & \multicolumn{2}{|c|}{ Atomic } & \multicolumn{2}{|c|}{ Coordinates (Angstroms } \\
\hline & & & Type & $X$ & $\mathrm{Z}$ \\
\hline 1 & 16 & 0 & -1.571677 & -1.104771 & 0.497743 \\
\hline 2 & 6 & 0 & -3.033237 & -0.365027 & 0.074537 \\
\hline 3 & 6 & 0 & -2.910201 & 0.957885 & -0.436841 \\
\hline 4 & 6 & 0 & -1.622252 & 1.419512 & -0.377325 \\
\hline 5 & 6 & 0 & -0.677783 & 0.433620 & 0.137753 \\
\hline 6 & 6 & 0 & 0.677715 & 0.433536 & -0.134109 \\
\hline 7 & 6 & 0 & 1.623606 & 1.418633 & 0.379262 \\
\hline 8 & 6 & 0 & 2.911873 & 0.957250 & 0.433459 \\
\hline 9 & 6 & 0 & 3.033159 & -0.365631 & -0.078954 \\
\hline 10 & 16 & 0 & 1.570285 & -1.105474 & -0.496363 \\
\hline 11 & 1 & 0 & -3.970704 & -0.899780 & 0.223165 \\
\hline 12 & 1 & 0 & -3.753783 & 1.515321 & -0.832794 \\
\hline 13 & 1 & 0 & -1.301458 & 2.398567 & -0.721710 \\
\hline 14 & 1 & 0 & 1.303959 & 2.397279 & 0.725865 \\
\hline 15 & 1 & 0 & 3.756829 & 1.514346 & 0.826944 \\
\hline 16 & 1 & 0 & 3.970138 & -0.900493 & -0.230250 \\
\hline
\end{tabular}

$\mathrm{HF}=-1104.0825318$ hartree 
Table S29. Cartesian Coordinates from the Optimized Structure of bithiophene dication $\left(C_{b}-C_{a}-C_{a}-C_{b}=\right.$ $\left.80^{\circ}\right)$ at B3LYP/6-31G(d)

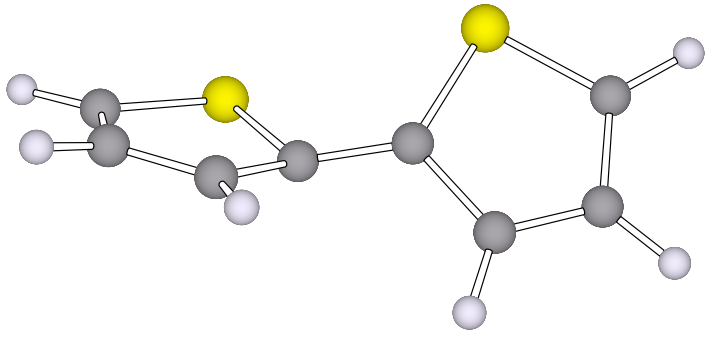

$\begin{array}{cccccc}1 & 16 & 0 & -1.537167 & -1.069344 & 0.570302 \\ 2 & 6 & 0 & -3.016560 & -0.390721 & 0.101834 \\ 3 & 6 & 0 & -2.920143 & 0.898132 & -0.491580 \\ 4 & 6 & 0 & -1.645479 & 1.398258 & -0.442671 \\ 5 & 6 & 0 & -0.679277 & 0.475470 & 0.144909 \\ 6 & 6 & 0 & 0.679223 & 0.475897 & -0.141318 \\ 7 & 6 & 0 & 1.647272 & 1.397163 & 0.444882 \\ 8 & 6 & 0 & 2.922237 & 0.897425 & 0.487645 \\ 9 & 6 & 0 & 3.016286 & -0.391611 & -0.106428 \\ 10 & 16 & 0 & 1.535452 & -1.070145 & -0.568758 \\ 11 & 1 & 0 & -3.942798 & -0.933305 & 0.288078 \\ 12 & 1 & 0 & -3.770454 & 1.408310 & -0.934416 \\ 13 & 1 & 0 & -1.345621 & 2.363059 & -0.842199 \\ 14 & 1 & 0 & 1.349010 & 2.361513 & 0.846675 \\ 15 & 1 & 0 & 3.774166 & 1.406994 & 0.928052 \\ 16 & 1 & 0 & 3.941782 & -0.934828 & -0.294520\end{array}$

$\mathrm{HF}=-1104.0740386$ hartree 
Table S30. Cartesian Coordinates from the Optimized Structure of bithiophene dication $\left(\mathrm{C}_{b}-\mathrm{C}_{\mathrm{a}}-\mathrm{C}_{\mathrm{a}}-\mathrm{C}_{\mathrm{b}}=\right.$ $\left.90^{\circ}\right)$ at B3LYP/6-31G(d)

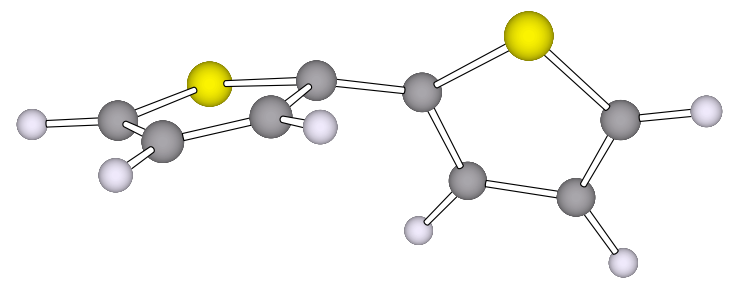

\begin{tabular}{ccccccc} 
Center & \multicolumn{2}{c}{ Atomic } & \multicolumn{2}{c}{ Atomic } & \multicolumn{3}{c}{ Coordinates (Angstroms) } \\
Number & Number & Type & X & Y & Z \\
-------------------------------------------- \\
1 & 16 & 0 & -1.951860 & -1.018148 & -0.615231 \\
2 & 6 & 0 & -3.098866 & -0.057998 & 0.164961 \\
3 & 6 & 0 & -2.565189 & 1.100424 & 0.800931 \\
4 & 6 & 0 & -1.234092 & 1.287680 & 0.532281 \\
5 & 6 & 0 & -0.655231 & 0.229032 & -0.273214 \\
6 & 6 & 0 & 0.654723 & -0.231093 & -0.272735 \\
7 & 6 & 0 & 1.235674 & -1.287960 & 0.533370 \\
8 & 6 & 0 & 2.566862 & -1.099718 & 0.800397 \\
9 & 6 & 0 & 3.098969 & 0.059008 & 0.163361 \\
10 & 16 & 0 & 1.950458 & 1.018194 & -0.615171 \\
11 & 1 & 0 & -4.152983 & -0.331046 & 0.134120 \\
12 & 1 & 0 & -3.158123 & 1.746942 & 1.441585 \\
13 & 1 & 0 & -0.639863 & 2.110187 & 0.919810 \\
14 & 1 & 0 & 0.642695 & -2.111125 & 0.921466 \\
15 & 1 & 0 & 3.160847 & -1.745294 & 1.441020 \\
16 & 1 & 0 & 4.152750 & 0.333351 & 0.132325
\end{tabular}

$\mathrm{HF}=-1104.0651978$ hartree 
Table S31. Cartesian Coordinates from the Optimized Structure of bithiophene dication $\left(\mathrm{C}_{b}-\mathrm{C}_{\mathrm{a}}-\mathrm{C}_{\mathrm{a}}-\mathrm{C}_{\mathrm{b}}=\right.$ $\left.100^{\circ}\right)$ at $\mathrm{B} 3 \mathrm{LYP} / 6-31 \mathrm{G}(\mathrm{d})$

\begin{tabular}{|c|c|c|c|c|c|}
\hline \multirow{2}{*}{$\begin{array}{l}\text { Center } \\
\text { Number }\end{array}$} & \multirow{2}{*}{\multicolumn{2}{|c|}{$\begin{array}{l}\text { Atomic } \\
\text { Number }\end{array}$}} & \multirow{2}{*}{$\begin{array}{l}\text { Atomic } \\
\text { Type }\end{array}$} & \multicolumn{2}{|c|}{ Coordinates (Angstrom } \\
\hline & & & & $X \quad Y$ & $\mathrm{Z}$ \\
\hline 1 & 16 & 0 & -1.945551 & $1 \quad-1.055947$ & -0.555769 \\
\hline 2 & 6 & 0 & -3.097542 & -0.062808 & 0.172299 \\
\hline 3 & 6 & 0 & -2.575935 & 1.138740 & 0.736308 \\
\hline 4 & 6 & 0 & -1.245245 & 1.317012 & 0.462185 \\
\hline 5 & 6 & 0 & -0.659192 & 0.210532 & -0.270936 \\
\hline 6 & 6 & 0 & 0.658887 & -0.211837 & -0.270562 \\
\hline 7 & 6 & 0 & 1.246062 & -1.317058 & 0.463220 \\
\hline 8 & 6 & 0 & 2.577074 & -1.138567 & 0.735550 \\
\hline 9 & 6 & 0 & 3.097632 & 0.063525 & 0.171410 \\
\hline 10 & 16 & 0 & 1.944701 & $1 \quad 1.055981$ & -0.555618 \\
\hline 11 & 1 & 0 & -4.148043 & $3-0.350964$ & 0.165249 \\
\hline 12 & 1 & 0 & -3.179541 & $1 \quad 1.820012$ & 1.328874 \\
\hline 13 & 1 & 0 & -0.658451 & $1 \quad 2.166888$ & 0.798329 \\
\hline 14 & 1 & 0 & 0.659865 & -2.166904 & 0.800521 \\
\hline 15 & 1 & 0 & 3.181464 & +-1.819456 & 1.327752 \\
\hline 16 & 1 & 0 & 4.147873 & 0.352640 & 0.164606 \\
\hline
\end{tabular}

$\mathrm{HF}=-1104.0745604$ hartree 
Table S32. Cartesian Coordinates from the Optimized Structure of bithiophene dication $\left(\mathrm{C}_{b}-\mathrm{C}_{\mathrm{a}}-\mathrm{C}_{\mathrm{a}}-\mathrm{C}_{\mathrm{b}}=\right.$ $\left.110^{\circ}\right)$ at $\mathrm{B} 3 \mathrm{LYP} / 6-31 \mathrm{G}(\mathrm{d})$

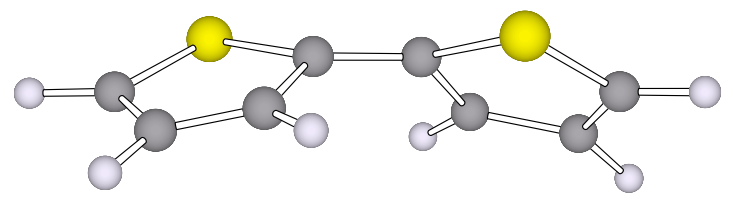

\begin{tabular}{|c|c|c|c|c|c|}
\hline \multirow{2}{*}{$\begin{array}{l}\text { Center } \\
\text { Number }\end{array}$} & \multirow{2}{*}{$\begin{array}{l}\text { Atomic } \\
\text { Number }\end{array}$} & \multicolumn{2}{|c|}{ Atomic } & \multicolumn{2}{|c|}{ Coordinates (Angstroms } \\
\hline & & & Type & $X$ & $\mathrm{Z}$ \\
\hline 1 & 16 & 0 & 1.936809 & 1.093089 & -0.493251 \\
\hline 2 & 6 & 0 & 3.099543 & 0.069502 & 0.171712 \\
\hline 3 & 6 & 0 & 2.594097 & -1.171041 & 0.663693 \\
\hline 4 & 6 & 0 & 1.261409 & -1.339705 & 0.395352 \\
\hline 5 & 6 & 0 & 0.662999 & -0.190921 & -0.258659 \\
\hline 6 & 6 & 0 & -0.662645 & 0.193821 & -0.258929 \\
\hline 7 & 6 & 0 & -1.263470 & 1.340345 & 0.396504 \\
\hline 8 & 6 & 0 & -2.596313 & 1.169989 & 0.662883 \\
\hline 9 & 6 & 0 & -3.099367 & -0.071695 & 0.170925 \\
\hline 10 & 16 & 0 & -1.934991 & -1.092895 & -0.493706 \\
\hline 11 & 1 & 0 & 4.146229 & 0.371076 & 0.181905 \\
\hline 12 & 1 & $\sigma$ & 3.212730 & -1.884353 & 1.200109 \\
\hline 13 & 1 & 0 & 0.684368 & -2.213673 & 0.682841 \\
\hline 14 & 1 & 0 & -0.688134 & 2.215353 & 0.684382 \\
\hline 15 & 1 & 0 & -3.216361 & 1.882117 & 1.199223 \\
\hline 16 & 1 & 0 & -4.145434 & -0.375399 & 0.181962 \\
\hline
\end{tabular}

$\mathrm{HF}=-1104.0827928$ hartree 
Table S33. Cartesian Coordinates from the Optimized Structure of bithiophene dication $\left(\mathrm{C}_{b}-\mathrm{C}_{\mathrm{a}}-\mathrm{C}_{\mathrm{a}}-\mathrm{C}_{\mathrm{b}}=\right.$ $\left.120^{\circ}\right)$ at $\mathrm{B} 3 \mathrm{LYP} / 6-31 \mathrm{G}(\mathrm{d})$

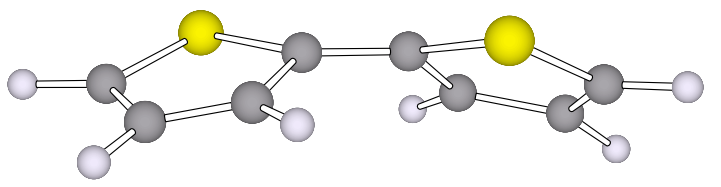

\begin{tabular}{|c|c|c|c|c|c|}
\hline \multirow{2}{*}{$\begin{array}{l}\text { Center } \\
\text { Number }\end{array}$} & \multirow{2}{*}{$\begin{array}{l}\text { Atomic } \\
\text { Number }\end{array}$} & \multicolumn{2}{|c|}{ Atomic } & \multicolumn{2}{|c|}{ Coordinates (Angstroms } \\
\hline & & & Type & $X$ & $\mathrm{Z}$ \\
\hline 1 & 16 & 0 & 1.925461 & 1.128459 & -0.427876 \\
\hline 2 & 6 & 0 & 3.104153 & 0.078671 & 0.163099 \\
\hline 3 & 6 & 0 & 2.618431 & -1.196603 & 0.583193 \\
\hline 4 & 6 & 0 & 1.281855 & -1.356981 & 0.331683 \\
\hline 5 & 6 & 0 & 0.666168 & -0.172818 & -0.238319 \\
\hline 6 & 6 & 0 & -0.665812 & 0.176113 & -0.238568 \\
\hline 7 & 6 & 0 & -1.284306 & 1.358053 & 0.332336 \\
\hline 8 & 6 & 0 & -2.620654 & 1.195117 & 0.583027 \\
\hline 9 & 6 & 0 & -3.103881 & -0.081225 & 0.162310 \\
\hline 10 & 16 & 0 & -1.923470 & -1.128300 & -0.428405 \\
\hline 11 & 1 & 0 & 4.146958 & 0.392828 & 0.184228 \\
\hline 12 & 1 & 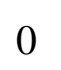 & 3.255329 & -1.937763 & 1.056488 \\
\hline 13 & 1 & 0 & 0.716774 & -2.252228 & 0.573753 \\
\hline 14 & 1 & 0 & -0.721219 & 2.254842 & 0.573488 \\
\hline 15 & 1 & 0 & -3.259173 & 1.934663 & 1.056653 \\
\hline 16 & 1 & 0 & -4.146260 & -0.396842 & 0.183314 \\
\hline
\end{tabular}

$\mathrm{HF}=-1104.0898882$ hartree 
Table S34. Cartesian Coordinates from the Optimized Structure of bithiophene dication $\left(\mathrm{C}_{b}-\mathrm{C}_{\mathrm{a}}-\mathrm{C}_{\mathrm{a}}-\mathrm{C}_{\mathrm{b}}=\right.$ $\left.130^{\circ}\right)$ at $\mathrm{B} 3 \mathrm{LYP} / 6-31 \mathrm{G}(\mathrm{d})$

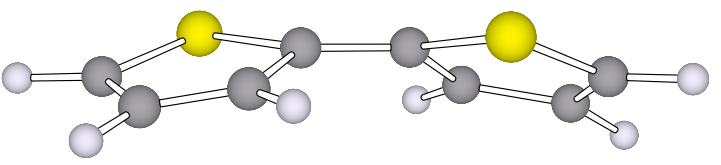

\begin{tabular}{|c|c|c|c|c|c|}
\hline \multirow{2}{*}{$\begin{array}{l}\text { Center } \\
\text { Number }\end{array}$} & \multirow{2}{*}{$\begin{array}{l}\text { Atomic } \\
\text { Number }\end{array}$} & \multicolumn{2}{|c|}{ Atomic } & \multicolumn{2}{|c|}{ Coordinates (Angstroms } \\
\hline & & & Type & $\mathrm{X}$ & $\mathrm{Z}$ \\
\hline 1 & 16 & 0 & 1.913073 & 1.160623 & -0.360475 \\
\hline 2 & 6 & 0 & 3.110715 & 0.089343 & 0.146104 \\
\hline 3 & 6 & 0 & 2.646308 & -1.215653 & 0.496259 \\
\hline 4 & 6 & 0 & & -1.369576 & 0.272001 \\
\hline 5 & 6 & 0 & 0.668700 & -0.156954 & -0.209384 \\
\hline 6 & 6 & 0 & -0.668409 & 0.159088 & -0.209695 \\
\hline 7 & 6 & 0 & -1.306 & 1.370295 & 0.272248 \\
\hline 8 & 6 & 0 & -2.647693 & 1.214675 & 0.496157 \\
\hline 9 & 6 & 0 & -3.110364 & -0.091242 & 0.146216 \\
\hline 10 & 16 & 0 & -1.911894 & -1.160433 & -0.361056 \\
\hline 11 & 1 & 0 & 4.150059 & 0.414514 & 0.170952 \\
\hline 12 & 1 & 0 & 3.303308 & -1.979543 & 0.900661 \\
\hline 13 & 1 & 0 & 0.752344 & -2.283153 & 0.471451 \\
\hline 14 & 1 & 0 & -0.755198 & 2.284940 & 0.470367 \\
\hline 15 & 1 & 0 & -3.305875 & 1.977647 & 0.900364 \\
\hline 16 & 1 & 0 & -4.149432 & -0.417310 & 0.171263 \\
\hline
\end{tabular}

$\mathrm{HF}=-1104.0958503$ hartree 
Table S35. Cartesian Coordinates from the Optimized Structure of bithiophene dication $\left(\mathrm{C}_{b}-\mathrm{C}_{\mathrm{a}}-\mathrm{C}_{\mathrm{a}}-\mathrm{C}_{\mathrm{b}}=\right.$ $\left.140^{\circ}\right)$ at $\mathrm{B} 3 \mathrm{LYP} / 6-31 \mathrm{G}(\mathrm{d})$

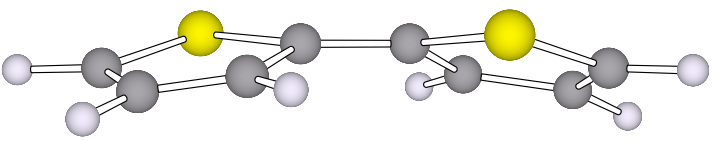

\begin{tabular}{|c|c|c|c|c|c|}
\hline \multirow{2}{*}{$\begin{array}{l}\text { Center } \\
\text { Number }\end{array}$} & \multirow{2}{*}{$\begin{array}{l}\text { Atomic } \\
\text { Number }\end{array}$} & \multicolumn{2}{|c|}{ Atomic } & \multicolumn{2}{|c|}{ Coordinates (Angstroms } \\
\hline & & & Type & $\mathrm{X}$ & $\mathrm{Z}$ \\
\hline 1 & 16 & 0 & 1.901163 & 1.188390 & -0.291126 \\
\hline 2 & 6 & 0 & 3.117690 & 0.100131 & 0.123854 \\
\hline 3 & 6 & 0 & 2.673678 & -1.229574 & 0.403820 \\
\hline 4 & 6 & 0 & 1.326462 & -1.378415 & 0.214492 \\
\hline 5 & 6 & 0 & 0.670516 & -0.143393 & -0.174523 \\
\hline 6 & 6 & 0 & -0.670402 & 0.144343 & -0.174993 \\
\hline 7 & 6 & 0 & -1.327180 & 1.378727 & 0.214535 \\
\hline 8 & 6 & 0 & -2.674275 & 1.229126 & 0.403882 \\
\hline 9 & 6 & 0 & -3.117566 & -0.100898 & 0.123824 \\
\hline 10 & 16 & 0 & -1.900630 & -1.188327 & -0.291355 \\
\hline 11 & 1 & 0 & 4.153957 & 0.435000 & 0.148864 \\
\hline 12 & 1 & 0 & 3.350092 & -2.011820 & 0.734077 \\
\hline 13 & 1 & 0 & 0.786472 & -2.307328 & 0.372337 \\
\hline 14 & 1 & 0 & -0.787771 & 2.308155 & 0.371426 \\
\hline 15 & 1 & 0 & -3.351124 & 2.010893 & 0.734370 \\
\hline 16 & 1 & 0 & -4.153695 & -0.436178 & 0.149275 \\
\hline
\end{tabular}

$\mathrm{HF}=-1104.1006896$ hartree 
Table S36. Cartesian Coordinates from the Optimized Structure of bithiophene dication $\left(\mathrm{C}_{b}-\mathrm{C}_{\mathrm{a}}-\mathrm{C}_{\mathrm{a}}-\mathrm{C}_{\mathrm{b}}=\right.$ $\left.150^{\circ}\right)$ at $\mathrm{B} 3 \mathrm{LYP} / 6-31 \mathrm{G}(\mathrm{d})$

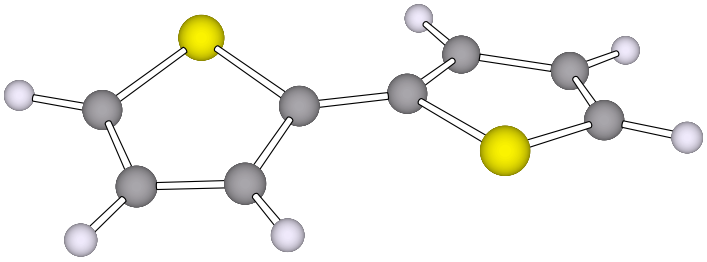

\begin{tabular}{|c|c|c|c|c|c|}
\hline \multirow{2}{*}{$\begin{array}{l}\text { Center } \\
\text { Numbel }\end{array}$} & \multirow{2}{*}{\multicolumn{2}{|c|}{$\begin{array}{l}\text { Atomic } \\
\text { Number }\end{array}$}} & & \multicolumn{2}{|c|}{ Coordinates (Angstroms } \\
\hline & & & Type & $\mathrm{X}$ & $\mathrm{Z}$ \\
\hline 1 & 16 & 0 & 1.890844 & 1.210969 & -0.219996 \\
\hline 2 & 6 & 0 & 3.124279 & 0.109476 & 0.097052 \\
\hline 3 & 6 & 0 & 2.697703 & -1.239416 & 0.306859 \\
\hline 4 & 6 & 0 & 1.345255 & -1.384198 & 0.158819 \\
\hline 5 & 6 & 0 & 0.671880 & -0.132105 & -0.134870 \\
\hline 6 & 6 & 0 & -0.671785 & 0.132628 & -0.135259 \\
\hline 7 & 6 & 0 & -1.345525 & 1.384219 & 0.159483 \\
\hline 8 & 6 & 0 & -2.698040 & 1.239298 & 0.306452 \\
\hline 9 & 6 & 0 & -3.124156 & -0.109948 & 0.097532 \\
\hline 10 & 16 & 0 & -1.890634 & -1.210929 & -0.220169 \\
\hline 11 & 1 & 0 & 4.158102 & 0.452063 & 0.118723 \\
\hline 12 & 1 & 0 & 3.390956 & -2.035924 & 0.558512 \\
\hline 13 & 1 & 0 & 0.815790 & -2.325073 & 0.276560 \\
\hline 14 & 1 & 0 & -0.816293 & 2.325374 & 0.276107 \\
\hline 15 & 1 & 0 & -3.391634 & 2.035820 & 0.557104 \\
\hline 16 & 1 & 0 & -4.157954 & -0.452621 & 0.119225 \\
\hline
\end{tabular}

$\mathrm{HF}=-1104.1044226$ hartree 
Table S37. Cartesian Coordinates from the Optimized Structure of bithiophene dication $\left(\mathrm{C}_{b}-\mathrm{C}_{\mathrm{a}}-\mathrm{C}_{\mathrm{a}}-\mathrm{C}_{\mathrm{b}}=\right.$ $\left.160^{\circ}\right)$ at $\mathrm{B} 3 \mathrm{LYP} / 6-31 \mathrm{G}(\mathrm{d})$

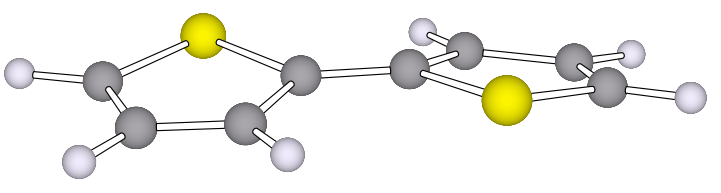

\begin{tabular}{|c|c|c|c|c|c|}
\hline \multirow{2}{*}{$\begin{array}{l}\text { Center } \\
\text { Number }\end{array}$} & \multirow{2}{*}{$\begin{array}{l}\text { Atomic } \\
\text { Number }\end{array}$} & \multicolumn{2}{|c|}{ Atomic } & \multicolumn{2}{|c|}{ Coordinates (Angstroms } \\
\hline & & & Type & $\mathrm{X}$ & $\mathrm{Z}$ \\
\hline 1 & 16 & 0 & 1.882890 & 1.227573 & -0.147384 \\
\hline 2 & 6 & 0 & 3.129656 & 0.116724 & 0.066369 \\
\hline 3 & 6 & 0 & 2.716425 & -1.245829 & 0.206370 \\
\hline 4 & 6 & 0 & & -1.387602 & 0.105291 \\
\hline 5 & 6 & 0 & 0.672721 & -0.123847 & -0.091620 \\
\hline 6 & 6 & 0 & -0.672695 & 0.124059 & -0.092047 \\
\hline 7 & 6 & 0 & -1.359758 & 1.387633 & 0.105399 \\
\hline 8 & 6 & 0 & -2.716539 & 1.245755 & 0.206320 \\
\hline 9 & 6 & 0 & -3.129597 & -0.116911 & 0.066681 \\
\hline 10 & 16 & 0 & -1.882808 & -1.227556 & -0.147550 \\
\hline 11 & 1 & 0 & 4.161733 & 0.464882 & 0.081885 \\
\hline 12 & 1 & 0 & 3.422733 & -2.052455 & 0.375872 \\
\hline 13 & 1 & 0 & 0.838120 & -2.337007 & 0.183786 \\
\hline 14 & 1 & 0 & -0.838360 & 2.337190 & 0.182873 \\
\hline 15 & 1 & 0 & -3.422941 & 2.052343 & 0.375602 \\
\hline 16 & 1 & 0 & -4.161659 & -0.465111 & 0.082358 \\
\hline
\end{tabular}

$\mathrm{HF}=-1104.1070724$ hartree 
Table S38. Cartesian Coordinates from the Optimized Structure of bithiophene dication $\left(\mathrm{C}_{b}-\mathrm{C}_{\mathrm{a}}-\mathrm{C}_{\mathrm{a}}-\mathrm{C}_{\mathrm{b}}=\right.$ $\left.170^{\circ}\right)$ at $\mathrm{B} 3 \mathrm{LYP} / 6-31 \mathrm{G}(\mathrm{d})$

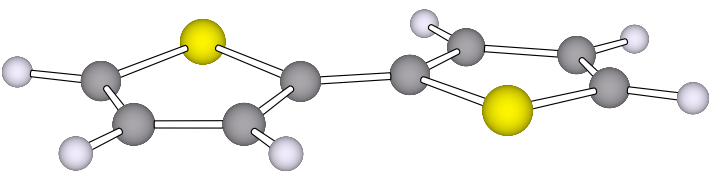

\begin{tabular}{|c|c|c|c|c|c|}
\hline \multirow{2}{*}{$\begin{array}{l}\text { Center } \\
\text { Number }\end{array}$} & \multirow{2}{*}{\multicolumn{2}{|c|}{$\begin{array}{l}\text { Atomic } \\
\text { Number }\end{array}$}} & & \multicolumn{2}{|c|}{ Coordinates (Angstrom } \\
\hline & & & Type & $\mathrm{X}$ & $\mathrm{Z}$ \\
\hline 1 & 16 & 0 & -1.877876 & -1.237685 & -0.073959 \\
\hline 2 & 6 & 0 & -3.133065 & -0.121358 & 0.034133 \\
\hline 3 & 6 & 0 & -2.728221 & 1.249424 & 0.103873 \\
\hline 4 & 6 & 0 & -1.368651 & 1.389474 & 0.052114 \\
\hline 5 & 6 & 0 & -0.673196 & 0.118832 & -0.046540 \\
\hline 6 & 6 & 0 & & -0.118802 & -0.047100 \\
\hline 7 & 6 & 0 & 1.368591 & -1.389432 & 0.052043 \\
\hline 8 & 6 & 0 & 2.728139 & -1.249465 & 0.104204 \\
\hline 9 & 6 & 0 & 3.133061 & 0.121267 & 0.033824 \\
\hline 10 & 16 & 0 & 1.877947 & 1.237721 & -0.073897 \\
\hline 11 & 1 & 0 & -4.164053 & -0.472976 & 0.042773 \\
\hline 12 & 1 & 0 & -3.442747 & 2.062035 & 0.189292 \\
\hline 13 & 1 & 0 & -0.852147 & 2.344065 & 0.091280 \\
\hline 14 & 1 & 0 & 0.852036 & -2.344035 & 0.090246 \\
\hline 15 & 1 & 0 & 3.442589 & -2.062083 & 0.190155 \\
\hline 16 & 1 & 0 & 4.164087 & 0.472774 & 0.042648 \\
\hline
\end{tabular}

$\mathrm{HF}=-1104.1086583$ hartree 
Table S39. Cartesian Coordinates from the Optimized Structure of bithiophene dication $\left(\mathrm{C}_{b}-\mathrm{C}_{\mathrm{a}}-\mathrm{C}_{\mathrm{a}}-\mathrm{C}_{\mathrm{b}}=\right.$ $\left.180^{\circ}\right)$ at $\mathrm{B} 3 \mathrm{LYP} / 6-31 \mathrm{G}(\mathrm{d})$

\begin{tabular}{|c|c|c|c|c|c|}
\hline \multirow{2}{*}{$\begin{array}{l}\text { Center } \\
\text { Number }\end{array}$} & \multirow{2}{*}{\multicolumn{2}{|c|}{$\begin{array}{l}\text { Atomic } \\
\text { Number }\end{array}$}} & \multirow{2}{*}{$\begin{array}{l}\text { Atomic } \\
\text { Type }\end{array}$} & \multicolumn{2}{|c|}{ Coordinates (Angstrom } \\
\hline & & & & $\begin{array}{ll}X & Y\end{array}$ & $\mathrm{Z}$ \\
\hline 1 & 16 & 0 & -1.876105 & 1.241098 & 0.000000 \\
\hline 2 & 6 & 0 & -3.134138 & 0.122858 & -0.000001 \\
\hline 3 & 6 & 0 & -2.732177 & -1.250648 & 0.000000 \\
\hline 4 & 6 & 0 & -1.371667 & -1.390048 & 0.000002 \\
\hline 5 & 6 & 0 & -0.673379 & -0.116916 & 0.000001 \\
\hline 6 & 6 & 0 & 0.673357 & 0.116794 & -0.000001 \\
\hline 7 & 6 & 0 & 1.371527 & 1.390009 & -0.000001 \\
\hline 8 & 6 & 0 & 2.732011 & 1.250774 & 0.000002 \\
\hline 9 & 6 & 0 & 3.134020 & -0.122777 & 0.000001 \\
\hline 10 & 16 & 0 & 1.876304 & -1.241137 & -0.000001 \\
\hline 11 & 1 & 0 & -4.164807 & 0.475527 & -0.000003 \\
\hline 12 & 1 & 0 & -3.449621 & -2.065158 & 0.000002 \\
\hline 13 & 1 & 0 & -0.856431 & -2.346129 & 0.000005 \\
\hline 14 & 1 & 0 & 0.856227 & 2.346055 & 0.000001 \\
\hline 15 & 1 & 0 & 3.449387 & 2.065340 & 0.000000 \\
\hline 16 & 1 & 0 & 4.164746 & -0.475289 & 0.000002 \\
\hline
\end{tabular}

$\mathrm{HF}=-1104.1091867$ hartree 
Table S40. Cartesian Coordinates from the Optimized Structure of terthiophene dication $\left(\mathrm{C}_{b}-\mathrm{C}_{\mathrm{a}}-\mathrm{C}_{\mathrm{a}}-\mathrm{C}_{\mathrm{b}}=\right.$ $\left.0^{\circ}, \mathrm{C}_{\mathrm{b}}{ }^{\prime}-\mathrm{C}_{\mathrm{a}}{ }^{\prime}-\mathrm{C}_{\mathrm{a}}{ }^{\prime}-\mathrm{C}_{\mathrm{b}}{ }^{\prime}=180^{\circ}\right)$ at $\mathrm{B} 3 \mathrm{LYP} / 6-31 \mathrm{G}(\mathrm{d})$

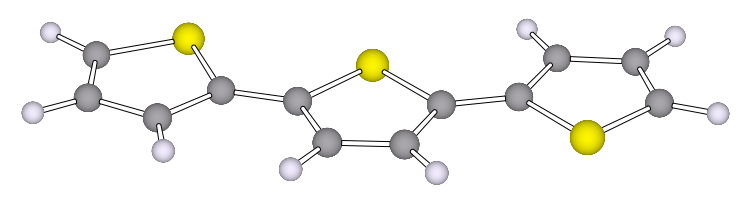

\begin{tabular}{|c|c|c|c|c|c|}
\hline Center & Atomic & & omic & Coordinates & (Angstrom \\
\hline Number & Numbe & & Type & $X$ & $\mathrm{Z}$ \\
\hline 1 & 16 & 0 & -3.229892 & 1.727143 & 0.000000 \\
\hline 2 & 6 & 0 & -4.843669 & 1.196325 & 0.000000 \\
\hline 3 & 6 & 0 & -4.991759 & -0.202387 & 0.000000 \\
\hline 4 & 6 & 0 & -3.774358 & -0.855581 & 0.000000 \\
\hline 5 & 6 & 0 & -2.656465 & 0.039794 & 0.000000 \\
\hline 6 & 6 & 0 & -1.316040 & -0.310856 & 0.000000 \\
\hline 7 & 6 & 0 & -0.795194 & -1.664138 & 0.000000 \\
\hline 8 & 6 & 0 & 0.560635 & -1.726266 & 0.000000 \\
\hline 9 & 6 & 0 & 1.204385 & -0.429119 & 0.000000 \\
\hline 10 & 16 & 0 & 0.000000 & 0.860213 & 0.000000 \\
\hline 11 & 1 & 0 & -5.646570 & 1.927889 & 0.000000 \\
\hline 12 & 1 & 0 & -5.959567 & -0.690832 & 0.000000 \\
\hline 13 & 1 & 0 & -3.671543 & -1.934624 & 0.000000 \\
\hline 14 & 1 & 0 & -1.439065 & -2.535510 & 0.000000 \\
\hline 15 & 1 & 0 & 1.122631 & -2.654304 & 0.000000 \\
\hline 16 & 6 & 0 & 2.564759 & -0.174985 & 0.000000 \\
\hline 17 & 6 & 0 & 3.233720 & 1.087888 & 0.000000 \\
\hline 18 & 6 & 0 & 4.609707 & 0.972500 & 0.000000 \\
\hline 19 & 6 & 0 & 5.030532 & -0.372658 & 0.000000 \\
\hline 20 & 16 & 0 & 3.765264 & -1.499524 & 0.000000 \\
\hline 21 & 1 & 0 & 2.707130 & 2.037038 & 0.000000 \\
\hline 22 & 1 & 0 & 5.304655 & 1.804547 & 0.000000 \\
\hline 23 & 1 & 0 & 6.058852 & -0.722637 & 0.000000 \\
\hline
\end{tabular}

$\mathrm{HF}=-1656.0037375$ hartree 
Table S41. Cartesian Coordinates from the Optimized Structure of terthiophene dication $\left(C_{b}-C_{a}-C_{a}-C_{b}=\right.$ $\left.30^{\circ}, \mathrm{C}_{\mathrm{b}}{ }^{\prime}-\mathrm{C}_{\mathrm{a}}{ }^{\prime}-\mathrm{C}_{\mathrm{a}}{ }^{\prime}-\mathrm{C}_{\mathrm{b}}{ }^{\prime}=180^{\circ}\right)$ at B3LYP/6-31G(d)

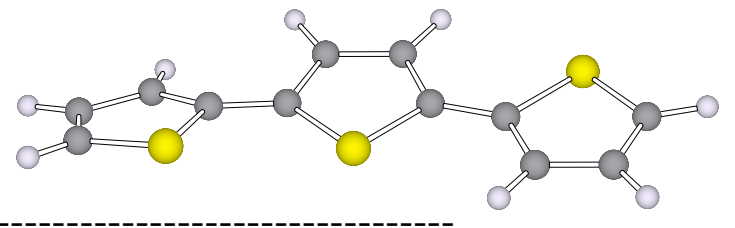

Center Atomic Atomic Coordinates (Angstroms)

Number Number Type $\mathrm{X} \quad \mathrm{Y} \quad \mathrm{Z}$

\begin{tabular}{cccccc}
1 & 16 & 0 & 3.391668 & -1.301255 & -0.305860 \\
2 & 6 & 0 & 4.933504 & -0.641989 & -0.022247 \\
3 & 6 & 0 & 4.925620 & 0.727653 & 0.292351 \\
4 & 6 & 0 & 3.649806 & 1.260378 & 0.272032 \\
5 & 6 & 0 & 2.643449 & 0.295434 & -0.053447 \\
6 & 6 & 0 & 1.271837 & 0.485961 & 0.045866 \\
7 & 6 & 0 & 0.591250 & 1.756256 & -0.110663 \\
8 & 6 & 0 & -0.761688 & 1.648327 & -0.152146 \\
9 & 6 & 0 & -1.242450 & 0.289998 & -0.012715 \\
10 & 16 & 0 & 0.111939 & -0.832990 & 0.146677 \\
11 & 1 & 0 & 5.810903 & -1.275697 & -0.113392 \\
12 & 1 & 0 & 5.827318 & 1.284118 & 0.521834 \\
13 & 1 & 0 & 3.422717 & 2.295128 & 0.504128 \\
14 & 1 & 0 & 1.129489 & 2.690898 & -0.219543 \\
15 & 1 & 0 & -1.429993 & 2.491163 & -0.294559 \\
16 & 6 & 0 & -2.560983 & -0.128230 & 0.018002 \\
17 & 6 & 0 & -3.069990 & -1.457094 & 0.154633 \\
18 & 6 & 0 & -4.449492 & -1.512285 & 0.149759 \\
19 & 6 & 0 & -5.032338 & -0.236378 & 0.009087 \\
20 & 16 & 0 & -3.915323 & 1.031241 & -0.116463 \\
21 & 1 & 0 & -2.431086 & -2.329291 & 0.251230 \\
22 & 1 & 0 & -5.037148 & -2.418767 & 0.240515 \\
23 & 1 & 0 & -6.095899 & -0.017679 & -0.022958 \\
------------------------------159 \\
\hline
\end{tabular}

$\mathrm{HF}=-1656.0003108$ hartree 
Table S42. Cartesian Coordinates from the Optimized Structure of terthiophene dication $\left(\mathrm{C}_{b}-\mathrm{C}_{\mathrm{a}}-\mathrm{C}_{\mathrm{a}}-\mathrm{C}_{\mathrm{b}}=\right.$ $\left.60^{\circ}, \mathrm{C}_{\mathrm{b}}{ }^{\prime}-\mathrm{C}_{\mathrm{a}}{ }^{\prime}-\mathrm{C}_{\mathrm{a}}{ }^{\prime}-\mathrm{C}_{\mathrm{b}}{ }^{\prime}=180^{\circ}\right)$ at B3LYP/6-31G(d)

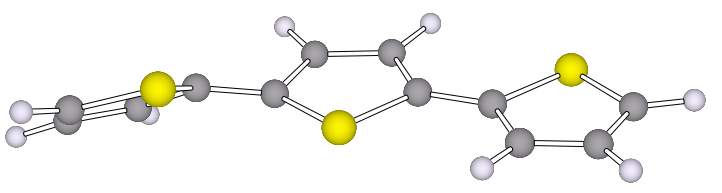

\begin{tabular}{|c|c|c|c|c|c|}
\hline \multirow{2}{*}{$\begin{array}{l}\text { Center } \\
\text { Number }\end{array}$} & \multirow{2}{*}{$\begin{array}{l}\text { Atomic } \\
\text { Number }\end{array}$} & \multicolumn{2}{|c|}{ Atomic } & \multicolumn{2}{|c|}{ Coordinates (Angstroms) } \\
\hline & & & Type & $X$ & Z \\
\hline 1 & 16 & 0 & 3.320592 & -1.185257 & -0.610515 \\
\hline 2 & 6 & 0 & 4.869328 & -0.732093 & -0.063217 \\
\hline 3 & 6 & 0 & 4.906050 & 0.515853 & 0.575012 \\
\hline 4 & 6 & 0 & 3.672652 & 1.146415 & 0.563091 \\
\hline 5 & 6 & 0 & 2.651741 & 0.381501 & -0.083776 \\
\hline 6 & 6 & 0 & 1.278026 & 0.576328 & 0.095957 \\
\hline 7 & 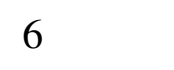 & 0 & 0.573769 & 1.807078 & -0.200768 \\
\hline 8 & 6 & 0 & -0.775750 & 1.662248 & -0.280069 \\
\hline 9 & 6 & 0 & -1.230296 & 0.313689 & -0.025710 \\
\hline 10 & 16 & 0 & & -0.760085 & 0.252833 \\
\hline 11 & 1 & 0 & 5.717869 & -1.381858 & -0.256453 \\
\hline 12 & 1 & 0 & 5.805331 & 0.927151 & 1.019729 \\
\hline 13 & 1 & & 3.476989 & 2.110163 & 1.022224 \\
\hline 14 & 1 & 0 & 1.102284 & 2.736452 & -0.385063 \\
\hline 15 & 1 & 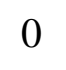 & -1.453609 & 2.468404 & -0.541122 \\
\hline 16 & 6 & U & -2.538614 & -0.131139 & 0.035548 \\
\hline 17 & 6 & 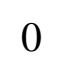 & -3.017234 & -1.455502 & 0.287424 \\
\hline 18 & 6 & 0 & -4.394438 & -1.543564 & 0.283354 \\
\hline 19 & 6 & 0 & -5.006501 & -0.298499 & 0.027775 \\
\hline 20 & 16 & 0 & -3.919791 & 0.979566 & -0.206870 \\
\hline 21 & 1 & 0 & -2.358019 & -2.299737 & 0.463192 \\
\hline 22 & 1 & 0 & -4.961625 & -2.452131 & 0.451181 \\
\hline 23 & 1 & 0 & -6.074924 & -0.109918 & -0.028600 \\
\hline
\end{tabular}

$\mathrm{HF}=-1655.9886134$ hartree 
Table S43. Cartesian Coordinates from the Optimized Structure of terthiophene dication $\left(\mathrm{C}_{b}-\mathrm{C}_{\mathrm{a}}-\mathrm{C}_{\mathrm{a}}-\mathrm{C}_{\mathrm{b}}=\right.$ $\left.90^{\circ}, \mathrm{C}_{\mathrm{b}}{ }^{\prime}-\mathrm{C}_{\mathrm{a}}{ }^{\prime}-\mathrm{C}_{\mathrm{a}}{ }^{\prime}-\mathrm{C}_{\mathrm{b}}{ }^{\prime}=180^{\circ}\right)$ at B3LYP/6-31G(d)

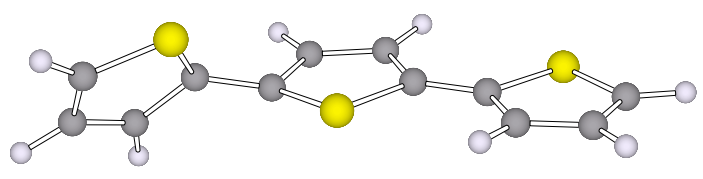

\begin{tabular}{|c|c|c|c|c|c|}
\hline \multirow{2}{*}{$\begin{array}{l}\text { Center } \\
\text { Number }\end{array}$} & \multirow{2}{*}{$\begin{array}{l}\text { Atomic } \\
\text { Number }\end{array}$} & \multicolumn{2}{|c|}{ Atomic } & \multicolumn{2}{|c|}{ Coordinates (Angstroms) } \\
\hline & & & Type & $\mathrm{X}$ & $\mathrm{Z}$ \\
\hline 1 & 16 & 0 & 3.225735 & -0.925163 & -0.933161 \\
\hline 2 & 6 & 0 & 4.771577 & -0.835039 & -0.215465 \\
\hline 3 & 6 & 0 & 4.865730 & 0.130742 & 0.790440 \\
\hline 4 & 6 & 0 & 3.698868 & 0.874552 & 0.916864 \\
\hline 5 & 6 & 0 & 2.675258 & 0.500838 & -0.000929 \\
\hline 6 & 6 & 0 & 1.282570 & 0.706333 & 0.195985 \\
\hline 7 & 6 & 0 & 0.548 & 1.883174 & -0.210274 \\
\hline 8 & 6 & 0 & -0.794164 & 1.688048 & -0.342439 \\
\hline 9 & 6 & 0 & -1.213117 & 0.344898 & -0.027340 \\
\hline 10 & 16 & 0 & 200839 & -0.665075 & 0.338910 \\
\hline 11 & 1 & 0 & 5.576602 & -1.460556 & -0.589371 \\
\hline 12 & 1 & 0 & 5.754187 & 0.278381 & 1.394333 \\
\hline 13 & 1 & 0 & 3.555250 & 1.658607 & 1.653706 \\
\hline 14 & 1 & 0 & 1.056248 & 2.818108 & -0.423428 \\
\hline 15 & 1 & 0 & -1.480774 & 2.452801 & -0.691019 \\
\hline 16 & 6 & 0 & -2.505907 & -0.140938 & 0.042156 \\
\hline 17 & 6 & 0 & -2.942609 & -1.470594 & 0.347004 \\
\hline 18 & 6 & 0 & -4.315026 & -1.604114 & 0.338401 \\
\hline 19 & 6 & 0 & -4.966577 & -0.390131 & 0.026334 \\
\hline 20 & 16 & 0 & -3.922767 & 0.913666 & $5 \quad-0.253375$ \\
\hline 21 & 1 & 0 & -2.256315 & -2.284030 & 0.560182 \\
\hline 22 & 1 & 0 & -4.854049 & -2.523268 & 0.538791 \\
\hline 23 & 1 & 0 & -6.040580 & -0.241481 & -0.045613 \\
\hline
\end{tabular}

$\mathrm{HF}=-1655.9680849$ hartree 
Table S44. Cartesian Coordinates from the Optimized Structure of terthiophene dication $\left(C_{b}-C_{a}-C_{a}-C_{b}=\right.$ $\left.120^{\circ}, \mathrm{C}_{\mathrm{b}}{ }^{\prime}-\mathrm{C}_{\mathrm{a}}{ }^{\prime}-\mathrm{C}_{\mathrm{a}}{ }^{\prime}-\mathrm{C}_{\mathrm{b}}{ }^{\prime}=180^{\circ}\right)$ at B3LYP/6-31G(d)

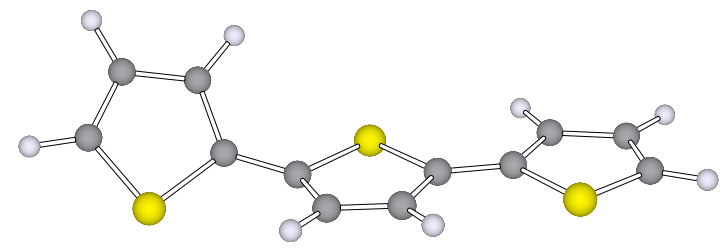

\begin{tabular}{|c|c|c|c|c|c|}
\hline \multirow{2}{*}{$\begin{array}{l}\text { Center } \\
\text { Number }\end{array}$} & \multirow{2}{*}{$\begin{array}{l}\text { Atomic } \\
\text { Number }\end{array}$} & \multicolumn{2}{|c|}{ Atomic } & \multicolumn{2}{|c|}{ Coordinates (Angstroms) } \\
\hline & & & Type & $\mathrm{X}$ & $\mathrm{Z}$ \\
\hline 1 & 16 & 0 & -3.896668 & -0.954951 & -0.563996 \\
\hline 2 & 6 & 0 & -5.054915 & 0.035652 & 0.192005 \\
\hline 3 & 6 & 0 & -4.513905 & 1.172984 & 0.810354 \\
\hline 4 & 6 & 0 & -3.146684 & 1.280497 & 0.613883 \\
\hline 5 & 6 & 0 & -2.604275 & 0.208878 & -0.155074 \\
\hline 6 & 6 & 0 & -1.264026 & -0.182443 & -0.237220 \\
\hline 7 & 6 & 0 & -0.709311 & -1.469589 & 0.117475 \\
\hline 8 & 6 & 0 & 0.642916 & -1.458942 & 0.264865 \\
\hline 9 & 6 & 0 & 1.248648 & -0.173683 & -0.003327 \\
\hline 10 & 16 & 0 & 0.005907 & 1.024418 & -0.386806 \\
\hline 11 & 1 & 0 & -6.106008 & -0.233006 & 0.143426 \\
\hline 12 & 1 & 0 & -5.109553 & 1.872148 & 1.386589 \\
\hline 13 & 1 & 0 & -2.531780 & 2.070413 & 1.032511 \\
\hline 14 & 1 & 0 & -1.336017 & -2.337801 & 0.291709 \\
\hline 15 & 1 & 0 & 1.218445 & -2.321951 & 0.583440 \\
\hline 16 & 6 & 0 & 2.599636 & 0.125566 & -0.013186 \\
\hline 17 & 6 & 0 & 3.231421 & 1.379498 & -0.286274 \\
\hline 18 & 6 & 0 & 4.608492 & 1.318206 & -0.218529 \\
\hline 19 & 6 & 0 & 5.068351 & 0.025310 & 0.109049 \\
\hline 20 & 16 & 0 & 3.838543 & -1.118288 & 0.331340 \\
\hline 21 & 1 & 0 & 2.677318 & 2.282302 & -0.523145 \\
\hline 22 & 1 & 0 & 5.278919 & 2.152667 & -0.390593 \\
\hline 23 & 1 & 0 & 6.106062 & -0.275223 & 0.223333 \\
\hline
\end{tabular}

$\mathrm{HF}=-1655.9883235$ hartree 
Table S45. Cartesian Coordinates from the Optimized Structure of terthiophene dication $\left(C_{b}-C_{a}-C_{a}-C_{b}=\right.$ $\left.150^{\circ}, \mathrm{C}_{\mathrm{b}}{ }^{\prime}-\mathrm{C}_{\mathrm{a}}{ }^{\prime}-\mathrm{C}_{\mathrm{a}}{ }^{\prime}-\mathrm{C}_{\mathrm{b}}{ }^{\prime}=180^{\circ}\right)$ at B3LYP/6-31G(d)

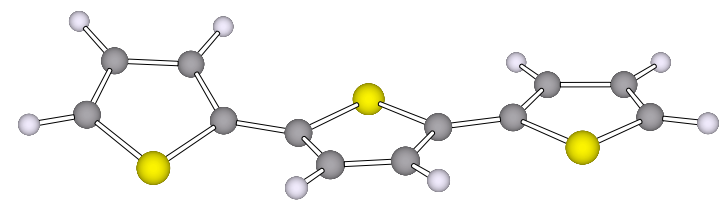

\begin{tabular}{|c|c|c|c|c|c|}
\hline \multirow{2}{*}{$\begin{array}{l}\text { Center } \\
\text { Number }\end{array}$} & \multirow{2}{*}{$\begin{array}{l}\text { Atomic } \\
\text { Number }\end{array}$} & \multicolumn{2}{|c|}{ Atomic } & \multicolumn{2}{|c|}{ Coordinates (Angstroms) } \\
\hline & & & Type & $\mathrm{X}$ & $\mathrm{Z}$ \\
\hline 1 & 16 & 0 & -3.876251 & -1.090363 & -0.280072 \\
\hline 2 & 6 & 0 & -5.072935 & 0.045896 & 0.112342 \\
\hline 3 & 6 & 0 & -4.574487 & 1.325639 & 0.421006 \\
\hline 4 & 6 & 0 & -3.198908 & 1.393808 & 0.303305 \\
\hline 5 & 6 & 0 & -2.608156 & 0.154725 & -0.090451 \\
\hline 6 & 6 & 0 & -1.261832 & -0.179997 & -0.131279 \\
\hline 7 & 6 & 0 & -0.687697 & -1.495017 & 0.061275 \\
\hline 8 & 6 & 0 & 0.668079 & -1.491189 & 0.142624 \\
\hline 9 & 6 & 0 & 1.257197 & -0.176876 & -0.005452 \\
\hline 10 & 16 & 0 & 0.001877 & 1.044353 & -0.224581 \\
\hline 11 & 1 & 0 & -6.118054 & -0.249406 & 0.096667 \\
\hline 12 & 1 & 0 & -5.213103 & 2.147396 & 0.724738 \\
\hline 13 & 1 & 0 & -2.616001 & 2.283729 & 0.517815 \\
\hline 14 & 1 & 0 & -1.298649 & -2.385913 & 0.161210 \\
\hline 15 & 1 & 0 & 1.263965 & -2.380682 & 0.318406 \\
\hline 16 & 6 & 0 & 2.606202 & 0.133098 & -0.006878 \\
\hline 17 & 6 & 0 & 3.225410 & 1.412451 & -0.157575 \\
\hline 18 & 6 & 0 & 4.604343 & 1.355612 & -0.116028 \\
\hline 19 & 6 & 0 & 5.077249 & 0.040240 & 0.067025 \\
\hline 20 & 16 & 0 & 3.857386 & -1.129123 & 0.189010 \\
\hline 21 & 1 & 0 & 2.662411 & 2.330830 & -0.290790 \\
\hline 22 & 1 & 0 & 5.266247 & 2.209054 & -0.209874 \\
\hline 23 & 1 & 0 & 6.118198 & -0.263214 & 0.132642 \\
\hline
\end{tabular}

$\mathrm{HF}=-1656.0004838$ hartree 
Table S46. Cartesian Coordinates from the Optimized Structure of terthiophene dication $\left(\mathrm{C}_{b}-\mathrm{C}_{\mathrm{a}}-\mathrm{C}_{\mathrm{a}}-\mathrm{C}_{\mathrm{b}}=\right.$ $\left.180^{\circ}, \mathrm{C}_{\mathrm{b}}{ }^{\prime}-\mathrm{C}_{\mathrm{a}}{ }^{\prime}-\mathrm{C}_{\mathrm{a}}{ }^{\prime}-\mathrm{C}_{\mathrm{b}}{ }^{\prime}=180^{\circ}\right)$ at $\mathrm{B} 3 \mathrm{LYP} / 6-31 \mathrm{G}(\mathrm{d})$

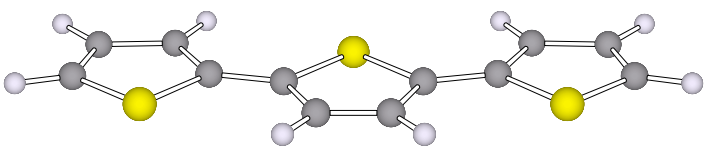

\begin{tabular}{|c|c|c|c|c|c|}
\hline \multirow{2}{*}{$\begin{array}{l}\text { Center } \\
\text { Number }\end{array}$} & \multirow{2}{*}{$\begin{array}{l}\text { Atomic } \\
\text { Number }\end{array}$} & \multicolumn{2}{|c|}{ Atomic } & \multicolumn{2}{|c|}{ Coordinates (Angstroms } \\
\hline & & & Type & $X$ & Z \\
\hline 1 & 16 & 0 & 3.866249 & -1.134972 & 0.000000 \\
\hline 2 & 6 & 0 & 5.081766 & 0.045300 & 0.000000 \\
\hline 3 & 6 & 0 & 4.603097 & 1.370881 & 0.000000 \\
\hline 4 & 6 & 0 & 3.223278 & 1.426678 & 0.000000 \\
\hline 5 & 6 & 0 & 2.609517 & 0.136324 & 0.000000 \\
\hline 6 & 6 & 0 & 1.261211 & -0.177357 & 0.000000 \\
\hline 7 & 0 & 0 & 0.678613 & -1.503583 & 0.000000 \\
\hline 8 & 6 & 0 & -0.678778 & -1.503496 & 0.000000 \\
\hline 9 & 6 & 0 & -1.261163 & -0.177136 & 0.000000 \\
\hline 10 & 16 & 0 & & 1.055612 & 0.000000 \\
\hline 11 & 1 & 0 & 6.124228 & -0.259916 & 0.000000 \\
\hline 12 & 1 & 0 & 5.261401 & 2.232211 & 0.000000 \\
\hline 13 & 1 & & 2.656229 & 2.352187 & 0.000000 \\
\hline 14 & 1 & & 1.282692 & -2.404784 & 0.000000 \\
\hline 15 & 1 & & -1.282980 & -2.404605 & 0.000000 \\
\hline 16 & 6 & 0 & -2.609510 & 0.136545 & 0.000000 \\
\hline 17 & 6 & & -3.223252 & 1.426877 & 0.000000 \\
\hline 18 & 6 & 0 & -4.603081 & 1.371079 & 0.000000 \\
\hline 19 & 6 & 0 & -5.081749 & 0.045505 & 0.000000 \\
\hline 20 & 16 & 0 & -3.866215 & -1.134758 & 0.000000 \\
\hline 21 & 1 & 0 & -2.656223 & 2.352400 & 0.000000 \\
\hline 22 & 1 & 0 & -5.261380 & 2.232412 & 0.000000 \\
\hline 23 & 1 & 0 & -6.124205 & -0.259725 & 0.000000 \\
\hline
\end{tabular}

$\mathrm{HF}=-1656.0045191$ hartree 
Table S47. Observed ${ }^{a}$ and Calculated ${ }^{b}{ }^{13} \mathrm{C}$ NMR Chemical Shifts $(\delta)$ for $\mathbf{1}(2 \mathrm{~T})^{2+}$ and $\mathbf{1}(3 \mathrm{~T})^{2+}$

\begin{tabular}{|c|c|c|c|}
\hline & $\mathrm{C}=\mathrm{C}$ & $\mathrm{CH}$ & $\mathrm{CH}_{2}$ \\
\hline $\mathbf{1}(2 \mathrm{~T})^{2+}$ obs. & 237.2166 .4155 .8141 .7 & 43.131 .6 & 27.123 .6 \\
\hline calcd. $(t)^{c}$ & 270.6160 .7150 .2143 .5 & $42.0 \quad 27.9$ & 24.222 .0 \\
\hline calcd. $(c)^{d}$ & 271.4159 .2152 .3143 .1 & 41.927 .9 & 24.122 .0 \\
\hline \multirow[t]{4}{*}{$\mathbf{1}(3 \mathrm{~T})^{2+}$ obs. } & 209.7164 .9162 .1160 .2141 .4140 .3 & $38.4 \quad 35.9 \quad 31.1$ & $\begin{array}{lll}26.1 & 25.7 & 24.4\end{array}$ \\
\hline & 242.6168 .4165 .6151 .0150 .5130 .8 & $\begin{array}{lll}36.9 & 31.1 & 27.7\end{array}$ & $\begin{array}{lll}23.2 & 23.2 & 23.2\end{array}$ \\
\hline & 242.9170 .5165 .7150 .7151 .3133 .9 & $36.931 .6 \quad 28.0$ & $23.4 \quad 23.4 \quad 23.2$ \\
\hline & 240.3168 .2164 .4150 .5148 .7130 .8 & $\begin{array}{lll}36.7 & 30.7 & 27.6\end{array}$ & $23.4 \quad 23.2 \quad 23.0$ \\
\hline
\end{tabular}

In $\mathrm{CH}_{2} \mathrm{Cl}_{2} .{ }^{b} \mathrm{GIAO} / \mathrm{HF} / 6-31+\mathrm{G}(\mathrm{d}) / / \mathrm{B} 3 \mathrm{LYP} / 6-31 \mathrm{G}(\mathrm{d})$.
transoid-transoid-Conformer. ${ }^{f}$ cisoid-transoid-Conformer. 